\title{
Álgebras de Hecke, Diagramas de Young e Invariantes de 3-variedades
}

Patrícia Fi. A. S. Nogueira

\author{
DISSERTAÇÃO APRESENTADA \\ $\mathrm{AO}$ \\ INSTITUTO DE MATEMÁTICA E ESTATÍSTICA \\ DA \\ UNIVERSIDADE DE SÃO PAULO \\ PARA \\ OBTENÇÃO DO GRAU DE MESTRE \\ EM \\ MATEMÁTICA
}

Área de Concentração: Topologia Algébrica

Orientador: Prof. Dr. Derek Hacon

Durante a elaboração deste trabalho a autora recebeu apoio financeiro do $\mathrm{CNPq}$

-São Paulo, março de 1998- 


\section{Álgebras de Hecke, Diagramas de Young e Invariantes de 3-variedades}

Este exemplar corresponde à redação final da dissertação devidamente corrigida e defendida por Patricia H. A. S. Nogueira e aprovada pela comissão julgadora.

São Paulo, 13 de março de 1998.

- Prof. Dr. Derek Hacon (Orientador) - PUC - RJ

- Prof. Dr. Frank M. Forger - IME - USP

- Prof. Dr. George Svetlichny - PUC - RJ. 


\section{Abstract}

This work intends to shows the construction of an invariant of closed oriented 3-manifolds starting from an invariant of framed oriented links, $\chi(L)$, and the decoration of knot's diagrams. The main ideas involved come from the so-called "Skein Theory" and from the relations among the skein space generated by the elementary $n$-braids, the Hecke Algebra $H_{n}$ and the Young diagrams.

\section{Resumo}

Este trabalho se propõe a apresentar a construção de um invariante de 3-variedades fechadas e orientáveis a partir de um invariante de links com frame orientados, $\chi(L)$, e decoração de diagramas de nós. Para isso, são usadas técnicas da chamada "Skein Theory" e as relações entre o "skein"espaço gerado pelas $n$-tranças elementares, a Álgebra de Hecke $H_{n}$ e os diagramas de Young. 
Afinal, para que servem livros sem figuras?

Alice 


\section{Agradecimentos}

À família maravilhosa que eu tenho, em especial aos meus pais Ana e Sylvio, aos meus avós Crizelídia e Mário, à minha tia Vera, ao meu irmão Sylvio e aos meus primos Beto e Ruth por todo apoio e carinho que recebi;

Aos amigões sempre pacientes com as minhas "crises pré-tese": Major, Liane, Daniel, Ronaldo, Alexandre, Fábio e Rosa;

Aos meus grandes colegas e amigos de graduação e mestrado: Luís, Pedro e principalmente o Raul pelas consultas e conselhos "via-embratel";

Aos professores que me passaram um pouco do seu amor pela Matemática, Zara Abud, Lucília Borsari, Hamilton Guidorizzi e Rubens Lintz;

Ao meu orientador Derek Hacon pelos toques sempre bem dados e pelo seu bom humor constante;

À Anna Aiston, da Universidade de Liverpool, pela atenção dispensada e por ter gentilmente cedido a mim os desenhos da sua tese, fazendo com que eu economizasse um tempo enorme;

E por final, ao maridão Mário por todo seu amor e companheirismo. 


\section{Índice}

1 Conceitos Básicos 3

1.1 Introdução . . . . . . . . . . . . . . . . . . . 3

1.2 Um breve estudo dos nós . . . . . . . . . . . . . . . 6

1.2.1 Equivalência de nós . . . . . . . . . . . . . 6

1.2 .2 Diagramas de nós . . . . . . . . . . . . . . 6

1.3 Invariantes polinomiais de nós . . . . . . . . . . . . . 10

1.3.1 O polinômio de Conway . . . . . . . . . . . . 10

1.3.2 O polinômio HOMFLY . . . . . . . . . . . . . . 11

1.4 Nós e Tranças . . . . . . . . . . . . . . . . . . . . . . . . 13

1.4.1 Nota: Movimentos de Markov . . . . . . . . . . . . . . 17

1.5 A álgebra de Hecke $H_{n} \quad \ldots \ldots \ldots \ldots \ldots$

1.6 Caracterização de uma 3-variedade através dos nós . . . . . . . . . . 19

1.6.1 Cirurgia ao longo de nós . . . . . . . . . . . . 19

1.6.2 Cirurgia ao longo de fitas . . . . . . . . . . . . 22

1.7 Decoração de nós . . . . . . . . . . . . . . . . . . . . . . 24

2 "Skein" Espaços 26 
2.1 Aplicações entre "Skein" Espaços . . . . . . . . . . . . . . . . . . 28

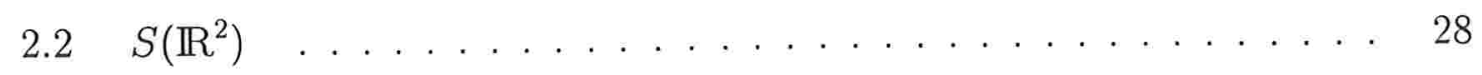

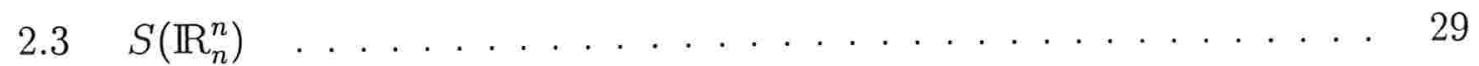

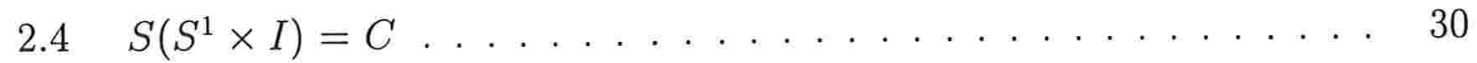

$3 \quad$ Idempotentes de $H_{n} \quad 33$

3.1 Diagramas de Young . . . . . . . . . . . . . . . . 33

3.2 Diagramas de Young e $\mathbb{C} S_{n} \ldots \ldots \ldots \ldots \ldots$

3.2 .1 Representações de $S_{n} \ldots \ldots \ldots \ldots . \ldots . \ldots . \ldots 37$

3.3 Construção dos idempotentes . . . . . . . . . . . . . . . . . 40

3.4 Especializando $H_{n} \quad \ldots \ldots \ldots \ldots \ldots$

3.5 Substituições no anel $\Lambda \ldots \ldots$. . . . . . . . . . 60

4 A Álgebra dos diagramas de Young $\quad 64$

4.1 O produto no conjunto dos diagramas . . . . . . . . . . . 64

$4.2 Y$ e $C^{+}$são álgebras isomorfas . . . . . . . . . . . . 65

4.2 .1 Nota: Fórmula de Giambelli . . . . . . . . . . . . . 66

4.3 Algumas relações entre os quase-idempotentes de $H_{n} \quad \ldots \ldots$. . . . . 72

5 Considerações finais: O invariante $T(L) \quad 103$

5.1 O espaço $R_{N} / \mathcal{I} \ldots \ldots \ldots \ldots \ldots \ldots$

$\begin{array}{ll}\text { Bibliografia } & 108\end{array}$ 


\section{Capítulo 1}

\section{Conceitos Básicos}

\subsection{Introdução}

O interesse na classificação dos nós nasce no século passado com a Teoria Atômica de Lord Kelvin, a qual associava cada tipo de nó a um elemento químico distinto. $\mathrm{Na}$ tentativa de obter uma tabela de elementos, P.G.Tait, em 1877, publicou o primeiro artigo contendo uma classificação empírica de nós.

Em 1928, J. Alexander descreve em seu trabalho um método que associa cada nó a um polinômio invariante por isotopia, o polinômio de Alexander, permanecendo este o único invariante polinomial por muitos anos.

A Teoria de Nós ganha maior fundamentação matemática com a publicação de "Knotentheorie" de K.Reidemeister em 1932, onde é mostrada a equivalência de nós através da equivalência de diagramas via certos movimentos chamados de movimentos de Reidemeister. A grande mudança só ocorre em 1970, quando Conway introduz novos métodos combinatórios, os quais deram origem ao que hoje chamamos de "Skein Theory", produzindo o polinômio de Conway.

Em 1984, uma pequena "revolução" acontece com o trabalho do matemático neo-zelandês Vaughan Jones, o qual produz um novo invariante polinomial de nós via Álgebra de Operadores, provocando uma onda de trabalhos na área. Apenas quatro meses depois é anunciada a descoberta de um novo invariante polinomial, o polinômio HOMFLY (nome originado das iniciais de seus autores: Hoste, Ocneanu, Millet, Freyd, Lickorish e Yetter), obtido de forma independente por grupos distintos (meses 
depois dois poloneses Prsytycki e Traczyc publicam trabalho em que apresentam o mesmo polinômio obtido independentemente dos outros grupos).

Vale observar que nenhum dos polinômios citados é um invariante completo de nós. Em particular, o HOMFLY é o mesmo para todo par de nós conhecidos como nós mutantes.

Também com o trabalho de Jones surge uma nova interação Matemática-Física, através, principalmente, do trabalho de Witten, no qual aparece uma interpretação do polinômio de Jones na Teoria Quântica de Campos, (a qual possui profundas ligações com a topologia de baixas dimensões [Atiyah]).

Em [RT2], Turaev e Reshetikhin deram um método para a construção de invariantes topológicos de 3-variedades a partir de Álgebras de Hopf modulares. Por outro lado, em [TW], Turaev e Wenzl provaram que a teoria de representações de $U_{q}(s l(N))$ (o chamado Grupo Quântico da álgebra de Lie $s l(N)$ ), quando consideradas em $q=r$-ésima raiz primitiva da unidade, gera uma álgebra de Hopf Modular e por conseguinte invariantes topológicos de 3-variedades (os sl(N)-invariantes quânticos).

Essa dissertação de mestrado é baseada no trabalho de Anna Aiston ([Anna]), onde é mostrada a construção de um invariante topológico de variedades de dimensão 3 , sem bordo, compactas, conexas e orientáveis, $\mathrm{T}(\mathrm{L})$, usando como base o Teorema de Kirby [Ki] e técnicas do que vem sendo chamada de "Skein Theory" (Teoria de "entrançamentos") introduzida por Conway em [C] e detalhada no trabalho de Lickorish [Li]. A autora também mostra a equivalência de $T(L)$ e o $s l(N)$-invariante quântico já citado.

Nosso objetivo é mostrar como se dá a construção do invariante $T(L)$ traçando sempre que possível um paralelo com o $s l(N)$-invariante quântico (sem contudo entrarmos efetivamente na construção deste último).

Como ponto de partida usaremos o polinômio "framed" HOMFLY $\chi(L)$ que é um invariante (por isotopia regular) de links orientados, mas que "infelizmente" não é invariante sob os movimentos de Kirby. Sendo assim, "complicamos" o diagrama original usando um processo conhecido como decoração de nós . A cada diagrama $P$ no anel $S^{1} \times I$, associamos um outro que é o resultado da decoração de $L$ por $P$, chamado satélite de $L$ e denotado por $L * P$. Esse processo tem a propriedade de que quando dois links $L_{1}$ e $L_{2}$ são equivalentes, os decorados com o mesmo padrão $P$ também o são. Logo, o valor de $\chi(L * P)$ é também um invariante de links associado a $L$. 
A idéia básica é que a partir de alguma escolha "esperta " de padrões no anel e após algumas correções, possamos encontrar um valor invariante pelos movimentos de Kirby e portanto um invariante topológico de 3-variedades.

Para isso, usaremos uma versão "diagramática" da álgebra de Hecke $H_{n}$ e provaremos que todo elemento de $C^{+}$é o fecho de um elemento de $H_{n}$ para algum $n$. Além disso, mostraremos que $C^{+}$é isomorfa como $\Lambda$-álgebra à $Y$, a álgebra dos diagramas de Young, que por sua vez aparecem na produção de idempotentes em $H_{n}$, cujos fechos irão gerar os padrões especiais que servirão de bloco para a construção do invariante $T(L)$.

A construção dos $\operatorname{sl}(N)$-invariantes quânticos baseia-se, por sua vez, na associação do diagrama $L$ a certas representações de $U_{q}(s l(N))$, através de um processo denominado coloração.

Por outro lado, temos que o anel das representações $U_{q}(s l(N))$ (com a multiplicação dada pelo produto tensorial), é isomorfo à álgebra $Y_{N}$, dos diagramas de Young com menos de $N$ linhas.

Lembrando que $C^{+} \simeq Y$, temos que, a grosso modo, "colorir" um diagrama $L$ com certas representações $V_{\lambda}$, onde $\lambda \in Y$, é o mesmo que "decorar" $L$ com um diagrama de Young $\lambda$ (resultado demonstrado em [Anna]), vindo portanto daí a relação entre o invariante $T(L)$ e o $s l(N)$-invariante quântico.

Por fim, gostariamos de ressaltar que a proposta inicial ao escolher esse tema foi mostrar que com técnicas mais elementares pode-se chegar aos mesmos resultados baseados na Teoria dos Grupos Quânticos, a qual exige maior embasamento matemático. Porém, no decorrer do trabalho, descobrimos que a abordagem feita em [Anna] contém alguns resultados cruciais (em especial a demonstração da invariância de $T(L)$ sob os movimentos de Fenn-Rourke) cujas demonstrações involvem essa teoria, e que portanto, sua construção dos "skein"-invariantes não está completamente divorciada dos invariantes quânticos.

Observamos que existe um outro trabalho realizado por Yokota em [Y], não abordado por essa dissertação, que não faz uso direto dessa conexão e que chega basicamente aos mesmos resultados de Aiston. 


\subsection{Um breve estudo dos nós}

\subsubsection{Equivalência de nós}

Definições 1.2.1 Dizemos que $L$ é um link de $k$ componentes se $L \subseteq \mathbb{R}^{3}$ ou $S^{3}$ é homeomorfo à união disjunta de $k$ cópias de $S^{1}$. Denotaremos $L=L_{1} \cup L_{2} \cup \ldots \cup L_{k}$. $L$ é dito orientado quando cada componente de $L$ possue uma orientação. Quando $k=1$, e portanto $L$ possue apenas uma componente, $L$ é chamado de nó.

Seja $I=[0,1]$ e $X$ uma espaço topológico.

Definição 1.2.1 Uma homotopia $h: X \times I \rightarrow X$ é chamada uma isotopia ambiente se $h_{0}$ é a aplicação identidade e $h_{t}$ é um homeomorfismo para todo $t \in I$.

Definições 1.2.2 Dois nós $K_{1}$ e $K_{2}$ são ditos isotópicos se existe uma isotopia ambiente (em $X=\mathbb{R}^{3}$ ) tal que $h_{1}\left(K_{1}\right)=K_{2}$. No caso de nós orientados, pede-se que a isotopia preserve a orientação. De forma análoga definimos links isotópicos.

A fim de evitarmos comportamentos patológicos (os chamados nós selvagens), vamos nos restringir nesse trabalho aos nós poligonais (em inglês, tames) que são os nós isotópicos a linhas poligonais simples e fechadas em $\mathbb{R}^{3}$ (ou $S^{3}$ ), ajustando assim as definições de isotopia ambiente para a categoria $P L$, ou "linear por partes".

\subsubsection{Diagramas de nós}

Podemos recuperar a descrição de um nó $K$ (ou link) apenas por uma projeção em um plano conveniente, $p: \mathbb{R}^{3} \rightarrow E$. Um ponto $P \in p(K) \subseteq E$ cuja pré-imagem sob a projeção contém mais de um ponto é chamado ponto múltiplo. Quando essa pré-imagem contém apenas dois pontos, este é chamado de ponto duplo.

Definição 1.2.2 Uma projeção $p: \mathbb{R}^{3} \rightarrow E$ de um nó $K$ é dita regular se:

(i) Existem finitos pontos múltiplos e todos estes são duplos; 
(ii) Nenhum vértice de $K$ (tomando-o como uma linha poligonal simples e fechada) é mapeado em um ponto duplo.

Novamente, observamos que a extensão aos links é imediata.

Proposição 1.2 .1 ([BZ], pg.8) O conjunto das projeções regulares é aberto e denso no espaço de todas as projeções.

Isto significa que, dado um nó $K$ (ou link), sempre podemos tomar uma projeção regular deste. Chamamos a imagem de $K$ por uma projeção regular de diagrama do nó $K$. Novamente, se o nó é orientado, pedimos que a projeção "carregue" essa informação.

Nesse caso, definimos o sinal de um cruzamento $c, \varepsilon(c)$, pela regra:
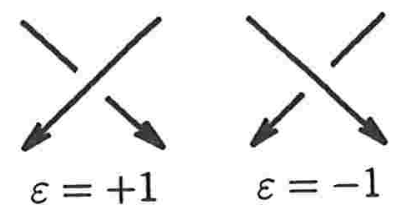

Sejam dois nós $K_{1}$ e $K_{2}$. Fixemos um diagrama de $K_{1} \cup K_{2}$. Definimos $l k\left(K_{1}, K_{2}\right)$ como:

$$
l k\left(K_{1}, K_{2}\right)=\sum_{c} \varepsilon(c)
$$

onde a soma é tomada sobre todos os cruzamentos $c$ de $K_{1}$ sobre $K_{2}$ e $\varepsilon(c)= \pm 1$ o sinal do cruzamento $c$.

Definição 1.2.3 Definimos o número de enlaçamento de $K_{1}$ e $K_{2}$ (em inglês linking number), como o número $l k\left(K_{1}, K_{2}\right)$.

OBS: Há várias outras definições (ver [Rol], pg.132) de número de enlaçamento, todas equivalentes a menos de um sinal.

É fácil ver que $l k\left(K_{1}, K_{2}\right)$ é inalterado se revertemos a orientação de ambas as componentes e que muda o sinal caso revertamos a orientação de apenas uma. Além disso, decorre da definição que quando $l k\left(K_{1}, K_{2}\right) \neq 0$ esses nós necessariamente são conectados (isto é, não existe uma isotopia ambiente que possibilita separá-los por dois abertos disjuntos). 
De forma análoga ao que fizemos, podemos definir o número de auto-enlaçamento, $w(L)$, (em inglês, writhe ) de um link orientado $L$ como:

$$
w(L)=\sum_{c} \varepsilon(c)
$$

onde $c$ percorre todos os cruzamentos de um diagrama de $L$.

A princípio, esses números não estão bem definidos, pois não sabemos se independem do diagrama escolhido. Vamos agora dar condições (necessárias e suficientes) para que dois diagramas distintos representem nós equivalentes.

Definição 1.2.4 Os movimentos de Reidemeister são os movimentos de caráter local, realizados em diagramas, descritos abaixo:

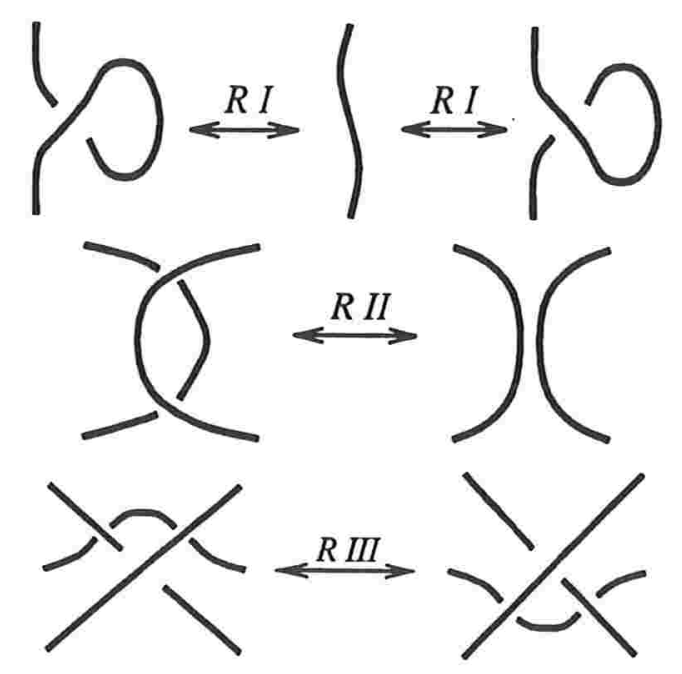

Teorema 1.2.1 ([PS], pg.11) Dois diagramas de links correspondem a links isotópicos se e somente se um pode ser obtido do outro através de uma sequência finita de movimentos de Redeimeister RI, RII e RIII.

Através desse resultado mostramos, por exemplo, que o número de enlaçamento é invariante por isotopia ambiente de links orientados: 
Proposição 1.2.2 O número de enlaçamento mantém-se inalterado sob os movimentos de Reidemeister, isto é, independe do diagrama escolhido. Além disso, vale que $l k\left(K_{1}, K_{2}\right)=l k\left(K_{2}, K_{1}\right)$

\section{Demonstração:}

O primeiro movimento, $R I$, envolve somente uma componente e portanto não afeta o número de enlaçamento. Sob RIII o número e tipos de cruzamentos não mudam e portanto também não afeta $l k$.

Já com o movimento $R I I$, dois cruzamentos são criados ou removidos, porém estes são sempre de sinais opostos, não afetando a soma final.

Para ver que $l k\left(K_{1}, K_{2}\right)=l k\left(K_{2}, K_{1}\right)$, basta observar que no segundo caso, existe uma projeção regular (um diagrama) onde todo cruzamento de $K_{1}$ sobre $K_{2}$ vira um cruzamento de $K_{2}$ sobre $K_{1}$ com o mesmo sinal.

Analogamente, escolhido um diagrama de um link orientado $L$, o número de auto-enlaçamento $w(L)$ é invariante pelos movimentos $R I I$ e RIII. Porém é claramente afetado por $R I$, pois um cruzamento é criado ou destruído, adicionado-se ou subtraindo-se 1 . Observe que $w(L)$ não é afetado pelo movimento $R^{\prime} I$ descrito abaixo:

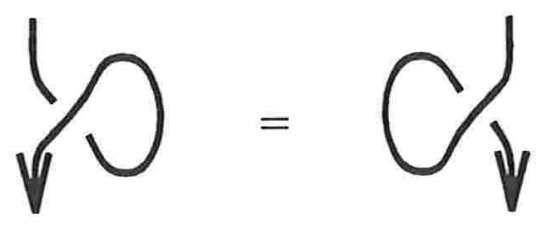

Definição 1.2.5 Uma sequência finita de movimentos $R I I, R I I I$ e $R^{\prime} I$ realizada sobre um diagrama é dita uma isotopia regular. Dizemos que dois diagramas são regularmente isotópicos se um pode ser obtido do outro através de isotopia regular.

Concluimos então que $w(L)$ é invariante sob isotopia regular. 


\subsection{Invariantes polinomiais de nós}

Para esse trabalho, somente interessarão os polinômios HOMFLY e o de Conway, este podendo ser obtido (transgredindo a ordem de cronológica) do primeiro.

\subsubsection{O polinômio de Conway}

O polinômio de Conway é um invariante de links orientados que pode ser definido de forma axiomática da seguinte maneira:

(A1) Para cada diagrama de link $L$ orientado, existe um polinômio $\nabla(L) \in \mathbb{C}[z]$, tal que:

$$
L \text { isotópico a } L^{\prime} \Rightarrow \nabla(L)=\nabla\left(L^{\prime}\right) \text {. }
$$

(A2) $L$ isotópico a $0 \Rightarrow \nabla(L)=1$.

(A3) Se $L_{+}, L_{-}$e $L_{0}$ são links que diferem apenas localmente pelos seguintes cruzamentos:

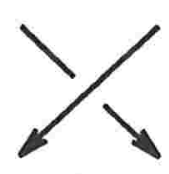

$L_{+}$

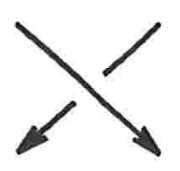

$L_{-}$

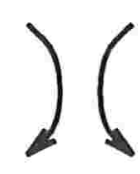

$L_{0}$

então $\nabla\left(L_{+}\right)-\nabla\left(L_{-}\right)=z \nabla\left(L_{0}\right)$.

Chamamos a relação dada em (A3), da "skein"-relação do polinômio de Conway.

OBS: A existência e várias outras propriedades desse polinômio podem ser encontradas em [Kau1]. 
Definição 1.3.1 um link é chamado de link desconexo, (em inglês, split link) se é equivalente a dois sublinks não vazios os quais se encontram contidos em regiões disjuntas do plano.

Proposição 1.3.1 Se L é um link desconexo, então $\nabla(L)=0$.

\section{Demonstração:}

Podemos assumir que $L$ tem um diagrama da forma:

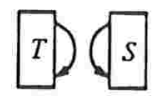

Sejam portanto $L_{+}=T S s \quad$ e $L_{-}=T s$

Fazendo uma rotação no espaço vemos que os links $L_{+}$e $L_{-}$são ambiente isotópicos e portanto $\nabla\left(L_{+}\right)=\nabla\left(L_{-}\right)$, por (A1). Logo, pela "skein"-relação dada em (A3) temos $\nabla(L)=0$.

Agora, citaremos mais uma propriedade do polinômio de Conway, que nos será útil posteriormente:

Proposição 1.3.2 ([Kau2], pg.82) O polinômio de Conway de um nó é dado por:

$$
\nabla(K)=1+z D(z)
$$

onde $D$ é outro polinômio em $z$. Quando $L$ é um link de mais de uma componente temos:

$$
\nabla(L)=z D^{\prime}(z)
$$

onde $D^{\prime}$ é um polinômio em $z$.

\subsubsection{O polinômio HOMFLY}

O polinômio HOMFLY, $P(L) \in \mathbb{C}\left[v^{ \pm 1}, z^{ \pm 1}\right]$ pode ser definido, a menos de um escalar, pela "skein"-relação:

$$
v^{-1} P\left(L_{+}\right)-v P\left(L_{-}\right)=z P\left(L_{0}\right)
$$

onde $L_{+}, L_{-}$e $L_{0}$ são os links que diferem locamente por um cruzamento segundo a regra descrita na subseção anterior. 
Normalizamos $P$ pedindo que o valor de $P$ no diagrama vazio seja 1 . Como uma consequência da "skein"-relação temos:

$$
P(L \cup O)=\frac{v^{-1}-v}{z} P(L)
$$

onde $L \cup O$ denota a união distante, isto é, união gerando um link desconexo, de $L$ unido com uma curva simples fechada. Esse polinômio é invariante sob os três movimentos de Reidemeister, ainda que não saia diretamente da "skein"-relação e é multiplicativo quando calculado em uma união distante, isto é, se $L_{1} \cup L_{2}$ é uma união distante, temos:

$$
P\left(L_{1} \cup L_{2}\right)=P\left(L_{1}\right) P\left(L_{2}\right) .
$$

Nota: Novamente, para maiores detalhes e demonstrações de propriedades desse polinômio, ver [Kau1].

No presente trabalho, nos interessará apenas links invariantes sob isotopia regular e portanto vamos modificar o polinômio $P$ para esse fim. Chamaremos esse novo polinômio de "framed" HOMFLY e o definiremos da seguinte maneira:

$$
\chi(L)=\left(x v^{-1}\right)^{w(L)} P(L)
$$

onde $w(L)$ é o número de auto-enlaçamento de $L$.

A relação abaixo passa a valer:

$$
\mathcal{X}(\curvearrowright)=\left(x v^{-1}\right) \mathcal{X}(\downarrow)
$$

E a "skein"-relação precisa ser ajustada para:

$$
x^{-1} \chi\left(L_{+}\right)-x \chi\left(L_{-}\right)=z \chi\left(L_{0}\right) .
$$

Definição 1.3.2 Seja $\Lambda$ o anel dado por:

$$
\Lambda=\mathbb{C}\left[x^{ \pm 1}, v^{ \pm 1}, s^{ \pm 1}, z, \delta\right] /\left\langle v^{-1}-v=\delta z\right\rangle
$$

onde $z=s-s^{-1}$. 
Observamos que $\chi(L)$ é um elemento desse anel. A variável $\delta$ é introduzida para posterior especialização de $\Lambda$, na qual será necessário "mandarmos" $z$ para 0 , enquanto que as variáveis $s$ e $s^{-1}$ são necessárias para descrição das relações em $H_{n}$, $\Lambda$-álgebra que definiremos no próximo capítulo. É imediato que ao fazermos $x=v=1$ recuperamos o valor do polinômio de Conway, fato que nos será útil posteriomente.

\subsection{Nós e Tranças}

Definição 1.4.1 Em $\mathbb{R}^{3}$, considere os pontos $A_{i}=(i, 0,0)$ e $B_{i}=(i, 0,1)$, onde $i=1, \ldots, n$. Uma curva simples unindo $A_{i}$ a $B_{j}$ é dita uma linha ascendente se cada plano horizontal a corta em exatamente um ponto. Uma n-trança, ou trança de $n$ linhas, é o conjunto de $n$ linhas ascendentes unindo os pontos $A_{i}{ }^{\prime} s$ aos $B_{j}{ }^{\prime} s$, duas a duas com interseção nula.

De forma análoga aos nós, definimos equivalência no conjunto das tranças via isotopia ambiente relativa à $\left\{A_{i}, B_{j} / i, j=1, \ldots, n\right\}$ e diagramas correspondentes a esses elementos, (sempre lembrando que estamos na categoria PL, ou linear por partes). Dois diagramas de tranças representam tranças equivalentes se forem obtidos um do outro por uma sequência finita de movimentos $R I I$ e $R I I I$ (e seus inversos), relativos aos pontos do bordo. Chamamos o conjunto das classes de equivalência de $n$-tranças de $B_{n}$, onde podemos definir um produto induzido pela operação geométrica "colar embaixo" tornando-o um grupo.

Teorema 1.4.1 ([A]) $B_{n}$ é isomorfo ao grupo abstrado gerado por:

$$
<\sigma_{i}, i=1, \ldots, n-1>
$$

com as relações:

$$
\begin{gathered}
\sigma_{i} \sigma_{j}=\sigma_{j} \sigma_{i},|i-j|>1 \\
\sigma_{i} \sigma_{i+1} \sigma_{i}=\sigma_{i+1} \sigma_{i} \sigma_{i+1}, i=1, \ldots, n-2
\end{gathered}
$$

onde $\sigma_{i}$ corresponde à $n$-trança elementar:

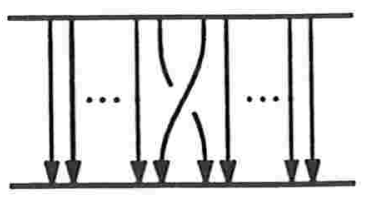


Obs: Graficamente, a equivalência pelo RIII é dada pela relação

$$
\sigma_{i} \sigma_{i+1} \sigma_{i}=\sigma_{i+1} \sigma_{i} \sigma_{i+1}
$$

como podemos ver abaixo:

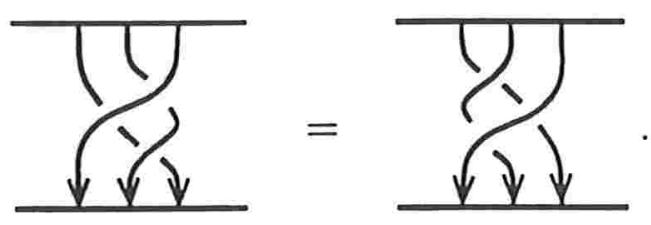

Nota:

(1) Para maiores detalhes consultar [BZ] e [Birman].

(2) Estaremos constantemente ao longo desse trabalho cometendo o abuso de hora tratar de uma trança como o objeto geométrico, hora como sua classe de equivalência.

Obs: Como o nosso interesse está focado em links orientados, vamos considerar a partir de agora tranças dotadas de orientação, a qual no nosso caso, por convenção será sempre "de cima para baixo".

Definição 1.4.2 O fecho de uma $n$-trança $\beta \in B_{n}$ é definido de forma natural como o link $\widehat{\beta}$ obtido unindo cada pontos $A_{i}$ com o ponto $B_{i}$ através de uma curva simples sem interseção com $\beta$ (excetuando-se obviamente os pontos $A_{i}, B_{i}$ ) e de tal forma que duas a duas possuam interseção vazia.

Assim como foi feito para nós, damos sinais a cada tipo de cruzamento. Numerando as linhas de uma $n$-trança de 1 a $n$, considere as $i$-ésima e $j$-ésima linhas tais que $i>j$. Quando a $i$-ésima linha cruza a $j$-ésima linha "por cima" dizemos que o cruzamento é positivo. Caso contrário, o cruzamento é considerado negativo.

Definição 1.4.3 Seja $\beta$ uma $n$-trança. Definimos o número de auto-enlaçamento $\boldsymbol{w}(\boldsymbol{\beta})$ de forma análoga ao que foi feito para diagramas de nós, isto é, fazendo-se a somatória dos sinais dos cruzamentos de $\beta$. 
Observe que esse número, por termos uma estrutura de grupo, é dado pela soma dos expoentes da trança $\beta$ escrita como uma palavra nas tranças geradoras $\sigma_{i}^{\prime} s$. A cada trança $\beta \in B_{n}$ associamos naturalmente uma permutação $\pi \in S_{n}$, definida por $\pi(i)=j$, se $\beta$ liga o ponto $A_{i}$ ao ponto $B_{j}$. Essa aplicação gera, de forma imediata, um epimorfismo $B_{n} \rightarrow S_{n}$, onde cada trança elementar $\sigma_{i}$ é levada na transposição $\phi_{i}=(i i+1)$. Lembramos que $S_{n}$ é gerado por:

$$
\left\{\phi_{i} / i=1, \ldots, n-1\right\}
$$

com as relações:

(i) $\phi_{i}^{2}=1$

(ii) $\phi_{i} \phi_{j}=\phi_{j} \phi_{i}$, se $|i-j|>1$

(iii) $\phi_{i} \phi_{i+1} \phi_{i}=\phi_{i+1} \phi_{i} \phi_{i+1}$

$S_{n}$ é, portanto, claramente obtido de $B_{n}$ adicionando-se a relação $\sigma_{i}^{2}=1$. Essa associação mostra-se importante em vários aspectos, entre eles:

Proposição 1.4.1 O fecho de uma n-trança é um nó se e somente se a permutação a ela associada é um n-ciclo.

\section{Demonstração:}

Lembrando a definição geométrica de uma trança, observamos primeiramente que o fecho de uma trança tem uma única componente se e somente se "saindo" de $A_{i}$ atingimos pelo fecho um outro ponto $A_{j}$, para quaisquer $i, j \in\{1, \ldots, n\}$. Isso significa portanto que a órbita do elemento $i$ pela permutação associada à trança contém $\{1, \ldots, n\}$, para qualquer $i$. Logo, essa permutação é um $n$-ciclo.

Definição 1.4.4 Para cada permutação $\pi \in S_{n}$, definimos uma $n$-trança $\omega_{\pi}$ chamada trança de permutação positiva, pelas seguintes propriedades:

(i) Todas as linhas são orientadas de cima para baixo;

(ii) Todos os cruzamentos são positivos;

(iii) A permutação associada a $\omega_{\pi}$ é a permutação $\pi$; 
(iv) Cada par de linhas cruzam-se no máximo uma vez.

Definimos uma trança de permutação negativa de maneira análoga, porém pedindo que todos os cruzamentos sejam negativos. Denotaremos essa trança por $\bar{\omega}_{\pi}$ e observamos que:

$$
\omega_{\pi}^{-1}=\bar{\omega}_{\pi^{-1}}
$$

Vemos a partir dessas propriedades, que dada uma permutação $\pi$ existe uma trança de permutação positiva $\omega_{\pi}$. Na verdade, essa relação é biunívoca:

Proposição 1.4.2 As tranças de permutação positiva são unicamente determinadas pelas propriedades (i)-(iv).

\section{Demonstração:}

Seja $\pi \in S_{n}$. Sejam $\omega_{\pi}$ e $\widetilde{\omega_{\pi}}$ duas $n$-tranças de permutação positiva associadas à $\pi$ (isto é, duas $n$-tranças que satisfazem as propriedades (i)-(iv)).

Nomeamos as linhas de ambas de 1 a $n$, de acordo com seu ponto inicial. Por construção, duas linhas distintas $i$ e $j$ (em ambas as tranças), têm no máximo um ponto de cruzamento, sendo que quando $i<j$, a $j$-linha passa à frente da $i$-linha (pois os cruzamentos são por hipótese positivos). Como consequência, podemos desenhar cada $i$-linha das duas tranças em planos distintos sucessivos.

Sendo assim, em cada plano, mantendo as extremidades fixas, pois por hipótese elas induzem a mesma permutação, podemos deformar a $i$-linha de $\omega_{\pi}$ na $i$-linha de $\widetilde{\omega_{\pi}}$, e portanto as duas tranças são equivalentes.

Obs: (I) É imediato que o resultado vale para as tranças de permutação negativa, pois foi usado apenas que os cruzamentos eram todos de mesmo sinal.

(II) Note que se $\omega_{\pi}$ é trança de permutação positiva, temos $w(\beta)=l(\pi)$, onde $l(\pi)$ é o tamanho da permutação $\pi$ (isto é, o tamanho da menor palavra nos geradores $\phi_{i}$ que representa $\pi$ ). 


\subsubsection{Nota: Movimentos de Markov}

Vimos que o fecho de uma trança gera um link. O Teorema de Alexander ([PS], pg.55) nos diz que todo link provém do fecho de uma trança. Markov, por sua vez, obteve condições puramente álgébricas que determinam quando duas tranças geram links equivalentes:

Teorema 1.4.2 Os fechos de duas tranças são equivalentes se e só se uma trança pode ser obtida da outra através de uma sequência finita dos movimentos abaixo:

$$
\begin{gathered}
M 1: \quad \beta \longleftrightarrow \alpha \beta \alpha^{-1} \alpha, \beta \in B_{n} \\
M 2: \quad \beta \longleftrightarrow \beta \sigma_{n}^{ \pm 1} \beta \in B_{n}
\end{gathered}
$$

onde a multiplicação $\beta \sigma_{n}$ faz sentido considerando a inclusão $B_{n} \subset B_{n+1}$ feita adicionando-se uma linha a cada $n$-trança $\beta$.

Esses movimentos são chamados movimentos de Markov e vê-se facilmente que eles não afetam o fecho de uma trança. Porém, a demonstração da outra implicação do teorema é extremamente complicada podendo ser encontrada em [Birman].

\subsection{A álgebra de Hecke $H_{n}$}

A $\mathbb{C}$-álgebra de Hecke $H_{n}(q)$ do tipo $A$ é definida pelos geradores:

$$
\left\{g_{1}, g_{2}, \ldots, g_{n-1}\right\}
$$

e relações :

(i) $g_{i}^{2}=(q-1) g_{i}+q$

(ii) $g_{i} g_{i+1} g_{i}=g_{i+1} g_{i} g_{i+1} \quad i=1, \ldots, n-2$

(iii) $g_{i} g_{j}=g_{j} g_{i}, \quad|i-j|>1$ 
Observe que há uma representação imediata de $B_{n}$ em $H_{n}(q)$ identificando $\sigma_{i}$ com o gerador $g_{i}$, na verdade essa álgebra é um quociente da álgebra de grupo de $B_{n}$. Usaremos ao longo desse trabalho uma outra versão de $H_{n}(q)$, a qual chamaremos $H_{n}$, dada pelos geradores:

$$
\left\{\sigma_{i} / i=1, \ldots, n-1\right\}
$$

e relações:

(H1) $\sigma_{i} \sigma_{j}=\sigma_{j} \sigma_{i},|i-j|>1$

(H2) $\sigma_{i} \sigma_{i+1} \sigma_{i}=\sigma_{i+1} \sigma_{i} \sigma_{i+1} \quad i=1, \ldots, n-2$

(H3) $x^{-1} \sigma_{i}-x \sigma_{i}^{-1}=z$

sobre o anel $\Lambda$ (ver 1.3.2), observando que podemos passar de uma para outra através da identificação: $x=s$ e $q=s^{2}$.

Observe que em $\Lambda$ podemos escrever a relação $(H 3)$ na forma:

$$
\left(\sigma_{i}-a\right)\left(\sigma_{i}-b\right)=0
$$

onde $a=-x s^{-1}$ e $b=x s$.

A aparente ambiguidade em usarmos o mesmo nome para as tranças elementares e para os geradores de $H_{n}$, desaparecerá no próximo capítulo onde veremos uma versão "geométrica" dessa álgebra através do que chamaremos "skein"-espaços.

\section{Nota:}

(1) Lembramos que a álgebra de grupo de $S_{n}, \mathbb{C} S_{n}$, é dada pelos geradores:

$$
\left\{\phi_{i}=(i i+1) / i=1, \ldots, n-1\right\}
$$

com as relações:

(i) $\phi_{i}^{2}=1$

(ii) $\phi_{i} \phi_{i+1} \phi_{i}=\phi_{i+1} \phi_{i} \phi_{i+1}, \quad i=1, \ldots, n-2$

(iii) $\phi_{i} \phi_{j}=\phi_{j} \phi_{i}, \quad|i-j|>1$ 
Logo, fazendo $q=1 \mathrm{em} H_{n}(q)$ recuperamos a álgebra $\mathbb{C} S_{n}$. Isto é, $H_{n}(q)$ pode ser vista como uma "deformação" de $\mathbb{C} S_{n}$. Wenzl, em [WZ], mostrou que quando $q \neq 0$ e $q$ não é uma $l$-ésima raiz da unidade para $l=2, \ldots, n, H_{n}(q) \simeq \mathbb{C} S_{n}$ como módulos.

(2) Pelo Teorema de Markov, vemos que, um funcional $f$ definido em $H_{n}(q)$ tal que $f\left(h_{1} \cdot h_{2}\right)=f\left(h_{2} \cdot h_{1}\right)$ e que seja invariante sob os movimentos de Markov, gera portanto um invariante de links. Essa, pois, é a base da construção do polinômio de Jones (ver [Jones]), utilizando-se o traço de Ocneanu em $H_{n}(q)$ para criar o invariante que acabou por provocar grandes mundanças no cenário da Teoria de Nós, como citamos na Introdução .

\subsection{Caracterização de uma 3-variedade através dos nós}

O objetivo dessa seção é apenas dar uma idéia de como um link $L$ pode caracterizar uma 3-variedade fechada (sem bordo e compacta), conexa e orientável $M$ através de um processo o qual damos o nome de cirurgia, justificando assim o interesse nos invariantes de nós para a construção de um invariante topológico de $M$. Uma exposição detalhada sobre o assunto pode ser encontrada em [Rol] e [PS].

\subsubsection{Cirurgia ao longo de nós}

Definições 1.6.1 Um toro sólido $V \subseteq S^{3}$ é uma 3-variedade (isto é, uma variedade de dimensão 3) homeomorfa a $S^{1} \times D^{2}$. Um homeomorfismo $h: S^{1} \times D^{2} \rightarrow V$ é chamado um referencial de $V$.

Uma curva simples fechada não trivialmente homotópica em $\partial V$ é chamada meridiano de $V$ se é homotopicamente trivial em $V$. Uma curva simples fechada em $\partial V$ é chamada longitude de $V$ se intercepta algum meridiano $V$ transversalmente em um único ponto.

Proposição 1.6.1 ([Rol], pg.29) 
(i) J é um meridiano de $V$ se e somente se $J$ é da forma $h\left(1 \times \partial D^{2}\right)$ para algum referencial $h$.

(ii) $K$ é uma longitude de $V$ se e somente se $K$ é da forma $h\left(S^{1} \times 1\right)$ para algum referencial $h$ de $V$.

Proposição 1.6.2 ([Rol], pg.30) Quaisquer dois meridianos são equivalentes em termos de isotopia ambiente em V. Por outro lado, existem infinitas classes de isotopia ambiente de longitudes em $V$.

Logo, a definição de meridiano é intrínseca, sendo que a de longitude involve uma escolha.

Proposição 1.6.3 ([Rol], pg.31) A menos de isotopia ambiente em $V$, existe uma única longitude l que é homologicamente trivial em $S^{3} \backslash V$, a qual chamaremos de de longitude preferencial.

Observamos que a classe de homotopia do meridiano, $m$ e a classe da longitude preferencial, $l$ são geradores do $\pi_{1}(\partial V)$.

Definição 1.6.1 Uma vizinhança tubular de um nó $K \subseteq S^{3}$ é uma imersão :

$$
f: K \times D^{2} \rightarrow S^{3}
$$

onde $f(x, 0)=x, \forall x \in K$. Uma vizinhança tubular de um nó é portanto um toro sólido.

Proposição 1.6.4 ([Rol], pg.133) Seja $V$ uma vizinhança tubular de um nó orientado $K$. Uma longitude preferencial $l$ de $V$ é caracterizada por $l k(l, K)=0$.

Considere um link $L=L_{1} \cup \ldots \cup L_{n}$ e vizinhanças tubulares $V_{i}$ para cada componente $L_{i}$, duas a duas sem interseção. Seja $h$ a união dos homeomorfismos $h_{i}: \partial V_{i} \rightarrow \partial V_{i}$ para $i=1, \ldots, n$. Considere $M$ o espaço obtido através da união:

$$
S^{3} \backslash\left(\operatorname{int}\left(V_{1}\right) \cup \ldots \cup \operatorname{int}\left(V_{n}\right)\right) \bigcup_{h}\left(V_{1} \cup \ldots \cup V_{n}\right)
$$

onde identificamos os bordos das vizinhanças $V_{i}{ }^{\prime} s$ através do homeomorfismo $h$. 
Proposição 1.6.5 ([Rol], pg.258) $M$ é uma 3-variedade sem bordo, compacta, conexa e orientável que depende somente a menos de homeomorfismo, da classe de homotopia de $h_{i}\left(m_{i}\right)$, onde $m_{i}$ é meridiano de $V_{i}$, para todo $i=1, \ldots, n$.

Dizemos que $M$ assim definida é obtida por uma cirurgia em $S^{3}$ ao longo do link L. A fim de caracterizarmos melhor esse procedimento devemos caracterizar portanto, segundo o resultado acima, a classe de homotopia das curvas $h_{i}\left(m_{i}\right.$.)

Seja $V$ um toro sólido, $m$ um meridiano de $V$ e $h: \partial V \rightarrow \partial V$ um homeomorfismo. Temos que $J=h(m)$ é um curva fechada simples em $\partial V$ que pode ser caracterizada como um elemento de $H_{1}(\partial V)$. Por outro lado, como $\pi_{1}(\partial V)=\mathbb{Z} \times \mathbb{Z}$, e portanto abeliano, podemos caracterizar $J$ como uma curva no grupo fundamental de $\partial V$. Logo, sendo [J] a classe de $J$ nesse grupo, temos que :

$$
[J]=p m+q l
$$

onde $m$ e $l$ são os geradores de $\pi_{1}(\partial V)$ e $p, q \in \mathbb{Z}$.

Proposição 1.6.6 ([PS], pg.45) Seja J curva simples fechada em $\partial V$. Nessas condições uma das afirmativas abaixo é válida:

(i) $\operatorname{mdc}(p, q)=1$;

(ii) $p=0$ e $q= \pm 1$

(iii) $p= \pm 1$ e $q=0$.

Voltemos à cirurgia ao longo de $L=L_{1} \cup \ldots \cup L_{n}$ um link orientado, considerando para cada componente a vizinhança tubular $V_{i}$, para todo $i=1, \ldots, n$. Para cada componente de $L$ existe, como já vimos, uma longitude preferencial $l_{i}$ orientada da mesma maneira que $L_{i}$ e um meridiano $m_{i}$ tal que $l k\left(L_{i}, m_{i}\right)= \pm 1$. Tomando $m_{i}$ e $l_{i}$ como os geradores do grupo fundamental de $\partial V_{i}$, como $J_{i}=h_{i}\left(m_{i}\right)$ é uma curva fechada simples, pela Proposição anterior, podemos caracterizar a classe de $J_{i}$ pela razão:

$$
r_{i}=\frac{p_{i}}{q_{i}}
$$

onde $\left[J_{i}\right]=p_{i} m_{i}+q_{i} l_{i}$.

Chamamos essa razão de coeficiente de cirurgia associado à componente $L_{i}$. Quando $q_{i}=0$, e portanto $p_{i}= \pm 1$, escrevemos $r_{i}=\infty$. Observe que nesse caso a cirurgia é trivial, isto é, a variedade obtida é novamente $S^{3}$. 


\section{Nota:}

(1) A escolha da orientação de $L_{i}$ é irrelevante, pois invertendo-se a orientação deste, a orientação de $h_{i}\left(m_{i}\right)$ também é invertida.

(2) Se $K$ é o nó trivial, a cirurgia com coeficiente $r=0$ resulta em $M \simeq S^{2} \times S^{1}$. Quando $r= \pm 1, \pm 1 / 2, \ldots, \pm 1 / n, \ldots, \infty$, temos $M \simeq S^{3}$.

Dizemos que a cirugia é inteira quando os coeficientes $r_{i}$ são números inteiros. Surpreendentemente, temos o seguinte resultado:

Teorema 1.6.1 ([PS], pg.104) Toda 3-variedade sem bordo, compacta, conexa $e$ orientável pode ser obtida através de uma cirurgia inteira em $S^{3}$ ao longo de um link $L$.

\subsubsection{Cirurgia ao longo de fitas}

Ao coeficiente de cirurgia associado a uma certa componente $L_{i}$ de um link $L$ damos o nome de framing de $L_{i}$. Vimos que, sob uma cirurgia inteira (a qual é sempre possível) ao longo de uma certa componente $K$ com framing $p$, o meridiano é mapeado na curva $J$, onde:

$$
[J]=p m+l .
$$

Nessa situação, $J$ e $K$ determinam uma fita em $S^{3}$ (como componentes do bordo desta), onde a informação $r=p$ traduz-se em:

$$
l k(J, K)=l k(p m+l, K)=p
$$

(o que decorre da escolha dos geradores $m$ e $l$ ) e portanto em $p$ "torcidas" na fita correspondente. Por sua vez, uma fita em $S^{3}$ pode ser transformada por isotopia em uma fita plana, usando-se o chamado "belt trick".

A idéia portanto é trabalhar com diagramas no plano representando essa fita, olhando apenas para o diagrama de uma das componentes do bordo, o qual chamaremos de diagrama de link com frame. Prova-se ([PS]) que mudanças nesse diagrama efetuadas por isotopia regular não afetam a fita original e portanto a cirurgia correspondente em $S^{3}$. Mais ainda, vale que: 
Teorema 1.6.2 ([PS], pg.124) Dois diagramas de links com frame correspondem a links isotópicos com mesmo framing (e portanto, produzem a mesma variedade) se $e$ somente se esses diagramas podem ser obtidos um do outro via isotopia regular.

Seja $M(L)$ a variedade obtida através de uma cirurgia em $S^{3}$ determinada pelo diagrama de link com frame L. O próximo resultado nos permite "decidir" quando duas cirurgias produzem, a menos de homeomorfismo, as mesmas variedades, olhando apenas para o diagrama $L$.

Teorema 1.6.3 [FR],[Ki], [Li1] Existe um homeomorfismo preservando orientação entre $M(L)$ e $M\left(L^{\prime}\right)$ se e somente se os diagramas de links com frame $L$ e $L^{\prime}$ podem ser obtidos um através do outro por isotopia regular (e isotopias do plano) e por uma sequência finita de movimentos de Fenn-Rourke, a saber:

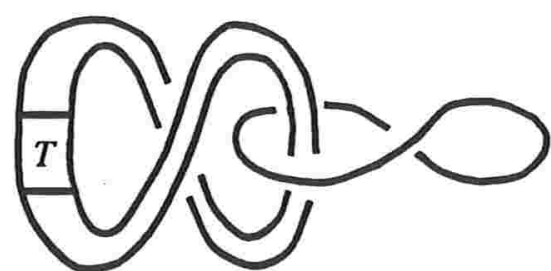

$L$

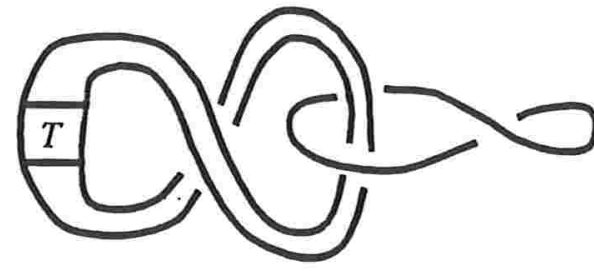

$L$

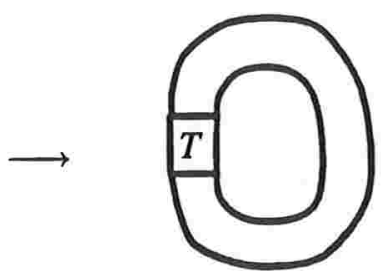

$\varphi_{+}(L)$

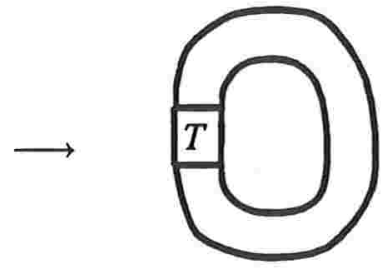

$\varphi_{-}(L)$

Este Teorema nos propicia, portanto, encontrar invariantes de 3-variedades fechadas, conexas e orientáveis produzindo invariantes de nós (por isotopia regular) que sejam também invariantes pelos movimentos de Fenn-Rourke. 


\subsection{Decoração de nós}

Suponha que ao calcularmos o polinômio HOMFLY de dois nós $L_{1}$ e $L_{2}$, os quais suspeitamos serem distintos, a resposta tenha sido a mesma. Podemos então tentar "complicá-los" obtendo dois outros nós $K_{1}$ e $K_{2}$ de maneira que se $L_{1}$ e $L_{2}$ forem equivalentes, necessariamente eles também o sejam e que seja possível a obtenção de repostas distintas das anteriores. Logo, se calcularmos os polinômios HOMFLY de $K_{1}$ e $K_{2}$ e obtivermos respostas distintas, teremos uma prova de que $L_{1}$ e $L_{2}$ não são equivalentes.

Uma maneira de fazermos essa "complicação" de nós que satisfaça as propriedades acima é o que chamamos de decoração de nós. Observamos novamente que um diagrama de nó nesse trabalho será sempre um diagrama orientado de nó com frame, e portanto a equivalência será em termos de isotopia regular. Seja $K$ um diagrama de nó. Lembremos que um nó com framing implica na escolha de uma curva paralela à ele, ou melhor, uma fita que é uma imersão do anel em $S^{3}$.

Definições 1.7.1 Seja $P$ um diagrama no anel. Considere a imagem de $\mathrm{P}$ pela imersão do anel na fita definida por $K$ e chame o seu diagrama no plano de $K * P$. Obtivemos então um diagrama de um nó $\widetilde{K}$ o qual chamaremos de nó satélite . Além disso, chamaremos $K$ de nó companheiro e $P$ de padrão.

\section{Exemplo:}
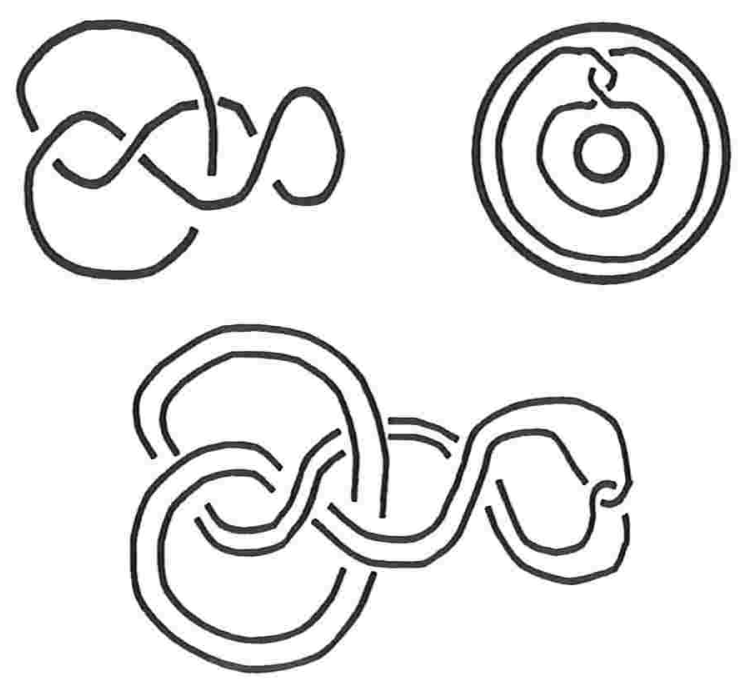

Satélite do nó figura-oito com framing -1. 
Se $K_{1}$ e $K_{2}$ são equivalentes, então $\widetilde{K_{1}}$ e $\widetilde{K_{2}}$ também o são. Sendo assim, $\widetilde{K}$ irá depender somente do nó $K$ e do padrão $P$.

Nota: Em [MS] há a demonstração da existência de pares de nós com o mesmo polinômio HOMFLY e uma decoração específica resultando em polinômios distintos. 


\section{Capítulo 2}

\section{"Skein" Espaços}

\section{Introdução:}

Observamos primeiramente que a partir de agora ao falarmos "links" estaremos nos referindo sempre ao diagrama orientado com frame deste. Estaremos também considerando a equivalência entre links dada por isotopia regular.

Vimos que a cada diagrama orientado $L$ de um link podemos associar um elemento do anel $\Lambda$ (def. 1.3.2) obtendo uma aplicação :

$$
\chi: D \rightarrow \Lambda
$$

onde $D$ é o conjunto das classes de diagramas de links orientados em $\mathbb{R}^{2}$ e $\chi(L)$ é o polinômio HOMFLY do diagrama $L$. Podemos, porém, trabalhar com mais estrutura, considerando um $\Lambda$-módulo livre tendo como base o conjunto $D$ e estendendo a aplicação $\chi$ para esse módulo, obtendo assim um funcional linear.

Vamos no entanto considerar $D$ como o conjunto das classes de diagramas e quocientar o $\Lambda$-módulo dos diagramas pelas "skein"-relações do polinômio HOMFLY, obtendo o $\Lambda$-módulo $S\left(\mathbb{R}^{2}\right)$, de modo que dois diagramas com o mesmo polinômio, sejam o mesmo elemento nesse módulo.

Passaremos portanto a fazer "contas" com diagramas de links e a fim de obtermos resultados interessantes e que nos levarão ao invariante de 3-variedades, vamos ter que considerar diagramas em outros espaços, definindo o nosso $\Lambda$-módulo de maneira análoga. 
Definições 2.0.2 Seja $F=\mathbb{R}^{2}$ ou $F \subseteq \mathbb{R}^{2}$, uma subvariedade compacta de dimensão dois equipada com um conjunto finito de pontos $\boldsymbol{P}$ em $\partial F$ orientados como pontos de "entrada" $\boldsymbol{P}_{\boldsymbol{E}}$ ou pontos de "saída" $\boldsymbol{P}_{\boldsymbol{S}}$.

Um diagrama em $\boldsymbol{F}$ é a interseção de $\boldsymbol{F}$ com um diagrama orientado $\boldsymbol{L}$ em $\mathbb{R}^{2}$, onde $\partial F \cap \boldsymbol{L}=\boldsymbol{P}$ e tal que $\partial \boldsymbol{F} \cap \boldsymbol{L}=\boldsymbol{P}$ não possua pontos de cruzamento de L. Além disso, a orientação de $\boldsymbol{L}$ em $\boldsymbol{\partial F} \cap \boldsymbol{D}=\boldsymbol{P}$ coincide com a dada aos pontos destacados.

Dois diagramas em $\boldsymbol{F}$ são equivalentes quando um pode ser obtido do outro através de uma sequência finita de isotopias de $\boldsymbol{F}$ e de isotopia regular, relativas a $\partial F$ (isto é, mantendo-o fixo). Seja $D_{F}$ o conjunto dessas classes de equivalência.

Definimos o "Skein" Espaço de $\boldsymbol{F}, \boldsymbol{S}(\boldsymbol{F})$, como o $\Lambda$-módulo com base em $D_{F}$ quocientado pelas relações locais:

(r1)

$$
\left.x^{-1} / y-x\right)=(
$$

$(\mathrm{r} 2)$

$$
\vartheta=\left(x v^{-1}\right) \downarrow
$$

$$
\text { (r3) } \quad D \bigsqcup \bigcirc=\frac{\left(v^{-1}-v\right)}{z} D
$$

Obs: A partir de agora não faremos distinção entre um diagrama e a sua classe. 


\subsection{Aplicações entre "Skein" Espaços}

Definição 2.1.1 Uma instalação $W$ de $F$ em $F^{\prime}$ é uma inclusão de $F$ em $F^{\prime}$ juntamente com um diagrama de curvas e arcos fixados em $F^{\prime} \backslash F$, tal que a fronteira desse diagrama fixado consiste exatamente nos pontos destacados nas fronteiras de $F$ e $F^{\prime}$, respeitando as orientações respectivas.

Qualquer diagrama $D$ em $F$ pode ser então estendido ao diagrama $W(D)$ em $F^{\prime}$.

Teorema 2.1.1 Uma instalação $W$ de $F$ em $F^{\prime}$ induz uma aplicação linear definida por:

$$
S_{W}: S(F) \rightarrow S\left(F^{\prime}\right)
$$

tal que $S_{W}(D)=W(D)$.

\section{Demonstração:}

Basta observar que como as relações são locais, uma coleção de diagramas em $S(F)$ que satisfazem determinadas relações continuarão a satisfazê-las em $S\left(F^{\prime}\right)$ quando for efetuada a instalação .

Corolário 2.1.1 Considere $F_{1}, F_{2}, \ldots, F_{n}$ com as respectivas instalações em $F S_{W_{1}}$, $S_{W_{2}}, \ldots, S_{W_{n}}$. Então essas instalações induzem uma aplicação multilinear:

$$
S_{W_{1}} \times S_{W_{2}} \times \cdots \times S_{W_{n}} \rightarrow S(F)
$$

Esse conceito será utíl para transformarmos alguns espaços em álgebras, como veremos a seguir.

\section{Exemplos:}

\section{$2.2 S\left(\mathbb{R}^{2}\right)$}

Pela Proposição anterior e pela existência e unicidade do polinômio HOMFLY, temos que a classe de qualquer diagrama $D$ no plano pode ser representada pelo elemento $\chi(D) . \phi \in S\left(\mathbb{R}^{2}\right)$ onde $\phi$ é a classe do diagrama vazio. 
Esse fato estabelece uma bijeção entre $S\left(\mathbb{R}^{2}\right)$ e $\Lambda$, que na verdade é um isomorfismo de $\Lambda$-módulos.

\section{$2.3 S\left(\mathbb{R}_{n}^{n}\right)$}

Definimos $\mathbb{R}_{n}^{n}$ como o retângulo $[0,1] \times[0,1]$ onde $\boldsymbol{P}_{E}=\{1\} \times\left\{\frac{1}{n+1}\right\}, \cdots,\{1\} \times$ $\left\{\frac{n}{n+1}\right\}$ e $P_{S}=\{0\} \times\left\{\frac{1}{n+1}\right\}, \cdots,\{0\} \times\left\{\frac{n}{n+1}\right\}$.

Um exemplo de um elemento desse espaço é:

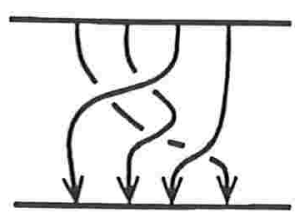

A instalação óbvia $W$ de "identificar $\{1\} \times[0,1]$ com $\{0\} \times[0,1]$, nos fornece uma aplicação bilinear $S\left(\mathbb{R}_{n}^{n}\right) \times S\left(\mathbb{R}_{n}^{n}\right) \rightarrow S\left(\mathbb{R}_{n}^{n}\right)$ a qual define um produto em $S\left(\mathbb{R}_{n}^{n}\right)$ tornando esse espaço uma álgebra.

\section{Exemplo:}

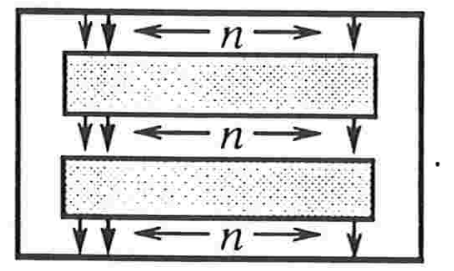

É fácil ver que há um homomorfismo do grupo de tranças $\boldsymbol{B}_{n}$ nessa álgebra, pois as relações entre os elementos desse grupo se traduzem na álgebra $S\left(\mathbb{R}_{n}^{n}\right)$ pelas relações $R I I$ e $R I I I$. Lembrando a conexão entre $B_{n}$ e a álgebra de Hecke $H_{n}$ (ver cap.1), temos o seguinte resultado:

Teorema 2.3.1 [MT] A álgebra $S\left(\mathbb{R}_{n}^{n}\right)$ e a álgebra de Hecke $H_{n}$ são isomorfas. Além disso, $S\left(\mathbb{R}_{n}^{n}\right)$ é gerada como álgebra pelas tranças elementares $\sigma_{i}$ orientadas. Como módulo tem como base as tranças de permutação positiva $\omega_{\pi}$ orientadas. 
Vamos a partir de agora tratar da álgebra $H_{n}$ na sua forma "pictórica" $S\left(\mathbb{R}_{n}^{n}\right)$. Considere portanto $h_{n} \in H_{n}$ e $h_{m} \in H_{m}$. Podemos "colar" esses dois elementos identificando as laterais dos retângulos $\mathbb{R}_{n}^{n}$ e $\mathbb{R}_{m}^{m}$, obtendo desse modo um representante de um elemento de $H_{n+m}$. Essa operação geométrica induz um homomorfismo de álgebras:

$$
\Theta: H_{n} \otimes_{\Lambda} H_{m} \rightarrow H_{n+m} .
$$

Abusando da notação vamos representar o elemento:

$$
\Theta\left(h_{n} \otimes h_{m}\right) \in H_{n+m}
$$

por $h_{n} \otimes h_{m}$. Observe que podemos incluir de forma homomórfica $H_{n}$ (ou $H_{m}$ ) em $H_{m+n}$ considerando os elementos $h_{n} \otimes 1^{\otimes m}$, onde $1^{\otimes m}$ é o elemento identidade de $H_{m}$ e portanto a trança trivial de $m$ linhas.

\section{$2.4 \quad S\left(S^{1} \times I\right)=C$}

Consideraremos $S^{1} \times I$ sem pontos destacados no bordo. Nesse caso espaço $C$ tornase uma álgebra com a multiplicação induzida pela instalação "inserir dentro" que é claramente comutativa em função dos movimentos RII e RIII (basta "passar" um diagrama "por baixo" do outro).

Considere $C^{+}$a sub-álgebra gerada pelos diagramas com orientação anti-horária. Sobre ela temos o seguinte resultado:

Teorema 2.4.1 [Turaev]: $C^{+}$é livremente gerado como álgebra pelos elementos $\varphi_{m}, m \in \mathbb{N}$, onde $\varphi_{m}$ é o fecho da trança $\sigma_{m-1} \sigma_{m-2} \ldots \sigma_{1}$.

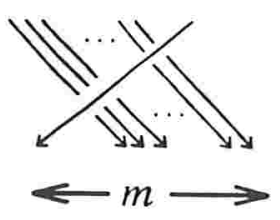

A trança $\sigma_{m-1} \sigma_{m-2} \ldots \sigma_{1}$ 
Definição 2.4.1 Seja $n \in \mathbb{N}$. Uma partição de $n$ é uma k-upla $\lambda=\left(\lambda_{1}, \lambda_{2}, \ldots, \lambda_{k}\right)$ onde $\lambda_{i} \in \mathbb{N}, \lambda_{1} \geq \lambda_{2} \geq \cdots \geq \lambda_{k}$ e $\sum_{i=0}^{k} \lambda_{i}=n$. Incluimos formalmente (0) como uma partição de $n=0$.

Corolário 2.4.1 Seja $C^{(n)}$ o submódulo de $C^{+}$gerado pelos elementos do tipo:

$$
\left(\varphi_{i_{1}}^{j_{1}}\right)\left(\varphi_{i_{2}}^{j_{2}}\right) \ldots\left(\varphi_{i_{p}}^{j_{p}}\right)
$$

onde $\sum_{k=0}^{p} i_{k} j_{k}=n$. Então:

(i) $C^{+}=\bigoplus_{n=0}^{\infty} C^{(n)} e c^{(n)} \cdot c^{(m)}=c^{(n+m)}$, onde $c^{(i)} \in C^{(i)}$

(ii) A dimensão de $C^{(n)}$ como $\Lambda$-módulo é o número de partições de $n$.

\section{Demonstração:}

Pelo Teorema 2.4.1, todo elemento de $C^{+}$é uma combinação $\Lambda$-linear de monômios em $\varphi_{m}, m \in \mathbb{N}$. Como esses elementos geram $C^{+}$livremente como álgebra, os monômios irão gerar $C^{+}$como $\Lambda$-módulo.

Seja $A_{n}=\left\{\left(\varphi_{i_{1}}^{j_{1}}\right)\left(\varphi_{i_{2}}^{j_{2}}\right) \ldots\left(\varphi_{i_{p}}^{j_{p}}\right) / \sum_{k=0}^{p} i_{k} j_{k}=n\right\}$. Como $C^{+}$é álgebra livre, $A_{n}$ é base de $C^{(n)}$. Observemos primeiramente, que pela comutatividade de $C^{+}$, podemos sempre supor $i_{1} \geq i_{2} \geq \ldots \geq i_{p}$. A cada $\left(\varphi_{i_{1}}^{j_{1}}\right) \ldots\left(\varphi_{i_{p}}^{j_{p}}\right)$ associamos a partição de $n$, $\lambda=\left(i_{1}, \ldots, i_{1}, \ldots, i_{p}, \ldots, i_{p}\right)$ onde cada $i_{k}$ aparece $j_{k}$ vezes. Reciprocamente, a partir de uma partição de $n$ (ordenada de forma decrescente) associamos de forma única um elemento de $A_{n}$. Logo, a dimensão de $C^{(n)}$ é o número de partições de $n$.

Resta verificarmos que a álgebra é graduada, observando que basta fazê-lo para os monômios. Sejam $c^{(n)}=\left(\varphi_{i_{1}}^{j_{1}}\right)\left(\varphi_{i_{2}}^{j_{2}}\right) \ldots\left(\varphi_{i_{p}}^{j_{p}}\right)$ e $c^{(m)}=\left(\varphi_{k_{1}}^{l_{1}}\right)\left(\varphi_{k_{2}}^{l_{2}}\right) \ldots\left(\varphi_{k_{q}}^{l_{q}}\right)$, tais que $\sum_{t=0}^{p} i_{t} j_{t}=n$ e $\sum_{t=0}^{q} k_{t} l_{t}=m$.

De fato,

$$
c^{(n)} \cdot c^{(m)}=\left(\varphi_{i_{1}}^{j_{1}}\right)\left(\varphi_{i_{2}}^{j_{2}}\right) \ldots\left(\varphi_{i_{p}}^{j_{p}}\right)\left(\varphi_{k_{1}}^{l_{1}}\right)\left(\varphi_{k_{2}}^{l_{2}}\right) \ldots\left(\varphi_{k_{q}}^{l_{q}}\right)
$$

onde $\sum_{t=0}^{p} i_{t} j_{t}+\sum_{t=0}^{q} k_{t} l_{t}=n+m$ e portanto $c^{(n)} \cdot c^{(m)} \in C^{(n+m)}$.

Obs: É fácil ver que $C^{(n)}$ é o espaço $\Lambda$-linear gerado pelo fecho das tranças de n linhas, porém podemos formalizar melhor essa afirmação usando o conceito de instalação. Definimos pois uma instalação de $\mathbb{R}_{n}^{n}$ no anel, a qual denominamos operação 
fecho, da seguinte maneira:

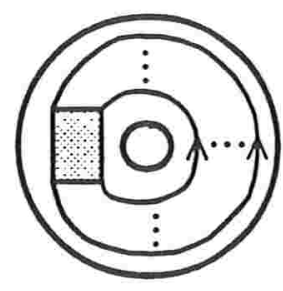

Essa operação induzirá portanto uma aplicação linear:

$$
\widehat{w}: S\left(\mathbb{R}_{n}^{n}\right) \rightarrow C^{+}
$$

que é não injetora, pois basta considerar as imagens dos elementos $\alpha$ e $\beta \alpha \beta^{-1}$, onde $\alpha$ e $\beta$ são tranças de n linhas. Observamos que, como as relações têm caráter local, $\widehat{w}\left(H_{n}\right)=C^{(n)}$.

OBS: Denotaremos, no decorrer desse trabalho, o fecho de um elemento $T \in S\left(\mathbb{R}_{n}^{n}\right)$ tanto por $\widehat{w}(T)$ como por $\widehat{T}$.

Como já foi dito na introdução o nosso objetivo é definir $\Omega_{r} \in C^{+}$, que possa produzir um valor $\chi\left(L * \Omega_{r}\right)$ o qual nos permita gerar um invariante de 3 -variedades. Logo, temos que estender a operação decoração para combinações lineares de diagramas, gerando desse modo uma aplicação composta:

$$
\chi(L *-): C^{+} \rightarrow \Omega
$$

definida por $\chi(L * P)=\chi\left(L * \sum_{i=1}^{n} \gamma_{i} P_{i}\right)=\sum_{i=1}^{n} \gamma_{i} \chi\left(L * P_{i}\right)$ onde $\gamma_{i} \in \Lambda$ e $P_{i}$ é um diagrama no anel.

É a partir dessa combinação de operações ( decoração + polinômio HOMFLY) que sairá o invariante procurado. 


\section{Capítulo 3}

\section{Idempotentes de $H_{n}$}

Introdução: Trabalhando na estrutura da álgebra de Hecke $H_{n}$ obteremos elementos (os idempotentes) cujos fechos serão a base da construção do nosso elemento especial $\Omega_{r}$. Para isso usaremos a relação entre a álgebra de grupo de $S_{n}, \mathbb{C} S_{n}$ e a álgebra de Hecke $H_{n}$. Além disso, estaremos sempre fazendo a identificação de $H_{n}$ com a álgebra $S\left(\mathbb{R}_{n}^{n}\right)$ dada pelo Teorema 2.3.1, tratando os elementos de $H_{n}$ como combinações lineares de diagramas.

\subsection{Diagramas de Young}

Definição 3.1.1 A cada partição de $n, \lambda=\left(\lambda_{1}, \lambda_{2}, \ldots, \lambda_{k}\right)$, ordenada de forma decrescente, podemos associar um diagrama formado por uma coleção de $n$ células arranjadas em linhas, de tal modo que existam $\lambda_{i}$ células na i-ésima linha. A esses diagramas damos o nome de diagramas de Young.

\section{Exemplo:}

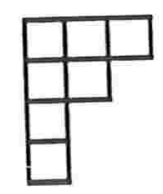

Diagrama de Young associado à partição $\mu=(3,2,1,1)$. 
Novamente, associamos a partição (0) ao diagrama vazio. Não faremos nesse trabalho distinção entre a partição e o diagrama associado.

Denotamos por $|\lambda|=\sum_{i=1}^{k} \lambda_{i}$ o tamanho da partição ou equivalentemente, o número de células do diagrama de Young associado.

Dado um diagrama de Young, o seu diagrama conjugado $\lambda^{v}$ é o diagrama cujas linhas têm o mesmo número de células das colunas de $\lambda$.

A um diagrama de Young $\lambda$ juntamente com uma numeração de 1 a $n$ associada às suas células denominamos de tableau de Young. Quando essa numeração é crescente em cima das linhas, da esquerda para direita, e em cima das colunas, de cima para baixo, dizemos que o tableau é padrão (ou standard).

\section{Exemplo:}

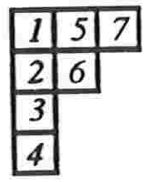

Tableau padrão associado ao diagrama $\mu=(3,2,1,1)$.

Dado um diagrama de Young $\lambda$ denotaremos cada célula por $\lambda_{i, j}$, onde $i$ representa a linha e $j$ a coluna onde a célula se encontra. Associaremos a cada $\lambda_{i, j}$ um número $h l\left(\lambda_{i, j}\right)$ (do inglês "hook length") que é que é definido como:

$$
h l\left(\lambda_{i, j}\right)=c_{A}\left(\lambda_{i, j}\right)+c_{D}\left(\lambda_{i, j}\right)+1
$$

onde $c_{A}\left(\lambda_{i, j}\right)$ e $c_{D}\left(\lambda_{i, j}\right)$ são o número de células abaixo e à direita de $\lambda_{i, j}$ respectivamente.

\section{Exemplo:}

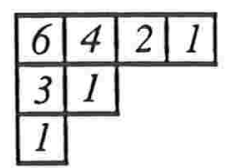

Diagrama que associa $h l\left(\lambda_{i, j}\right)$ à célula $\lambda_{i, j}$. 
Proposição 3.1.1 [FRT] Dado um diagrama de Young $\lambda$, o número de tableaux padrões $d_{\lambda}$ é dado pela seguinte fórmula:

$$
d_{\lambda}=\frac{n !}{\prod h l\left(\lambda_{i, j}\right)}
$$

onde $n$ é o número de células de $\lambda$.

\subsection{Diagramas de Young e $\mathbb{C} S_{n}$}

Faremos agora um pequeno resumo de alguns resultado em Teoria de Representação de Grupos Finitos, sempre tomando $\mathbb{C}$ como o corpo considerado (ou melhor, os resultados abaixo não são necessariamente válidos quando o corpo não é o dos complexos.)

Definições 3.2.1 Uma representação de um grupo finito $G$ em um espaço vetorial complexo de dimensão finita $V$ é um homomorfismo $\rho: G \rightarrow G L(V)$, onde $G L(V)$ é o grupo dos automorfismos de $V$. A dimensão de $V$ é chamada de grau da representação .

Dizemos que duas representações $\rho$ e $\phi$ são equivalentes quando existe $T \in$ $G L(V)$ tal que:

$$
\rho(g)=T^{-1} \phi(g) T, \forall g \in G
$$

Um $G$ - módulo é um espaço vetorial $V$ sobre $\mathbb{C}$ onde cada $g \in G$ define uma aplicação linear $v \mapsto g . v$ em $V$ satisfazendo:

$$
\begin{gathered}
\left(g_{1} g_{2}\right) \cdot v=\left(g_{1}\right) \cdot g_{2} \cdot v \\
e . v=v
\end{gathered}
$$

$\forall g_{1}, g_{2} \in G, \forall v \in V$

Naturalmente, um homomorfismo de $G$-módulos $V$ e $W$ é uma aplicação linear $T: V \rightarrow W$ que preserva a ação de $\mathrm{G}$, isto é:

$$
T(g \cdot v)=g \cdot T(v), \quad \forall v \in V, \forall g \in G
$$


Considerando a matriz da ação de $G$ em um $G$-módulo $V$, estabelecemos uma relação entre $V$ e uma representação de $G$. Reciprocamente, dada uma representação de $G$ em um espaço vetorial $V$ de dimensão finita, naturalmente podemos torná-lo um $G$-módulo. Na verdade, vale que:

Proposição 3.2.1 [Cohn] Para todo grupo finito $G$ existe uma bijeção natural entre as classes de equivalência de representações de $G$ e classes de isormofismo de G-módulos.

Definições 3.2.2 Um $G$-submódulo $W$, de um $G$-módulo $V$ é um subespaço vetorial de $V$ que é fechado sob a ação de $G$. Dizemos que um $G$-módulo $V$ é irredutível se é não nulo e se não possui submódulos além dos triviais. Caso contrário dizemos que $V$ é redutível.

Por sua vez, dizemos que uma representação é irredutível quando o $G$ módulo correspondente o for. Analogamente, definimos uma representação redutível.

Um $G$-módulo é dito completamente redutível se:

$$
V=U_{1} \oplus U_{2} \oplus \cdots \oplus U_{r}
$$

onde $U_{i}$ é um $G$-submódulo irredutível de $V$ para $i=1, \ldots, r$.

Teorema 3.2.1 ([JL], pg.74) Seja G grupo finito. Então todo G-módulo sobre $\mathbb{C}$ é completamente redutivel.

Definição 3.2.1 Seja $G=\left\{g_{1}, \ldots, g_{n}\right\}$ grupo finito. Considere o espaço vetorial sobre $\mathbb{C}$ com base $\left\{g_{1}, \ldots, g_{n}\right\}$, isto é, o espaço formado por elementos do tipo:

$$
\sum_{g \in G} \lambda_{g} g
$$

onde $\lambda_{g} \in \mathbb{C}, \forall g \in G$. Denotaremos esse espaço de $\mathbb{C} G$.

Podemos definir um produto nesse espaço tornando-o uma álgebra de forma natural:

$$
\left(\sum_{g \in G} \lambda_{g} g\right)\left(\sum_{h \in G} \mu_{h} h\right)=\sum_{g, h \in G} \lambda_{g} \mu_{h}(g h) .
$$


Chamamos $\mathbb{C} G$ a álgebra de grupo de $G$ sobre $\mathbb{C}$. $\mathbb{C} G$ visto como $\mathbb{C}$-espaço vetorial é em particular um $G$-módulo (sob a multiplicação à direita pelos elementos de $G$ ), o qual chamaremos de $G$-módulo regular. A representação obtida do $G$-módulo regular é dita representação regular de $G$. Observe que a dimensão de $\mathbb{C} G$ é o número de elementos desse grupo.

Pelo Teorema anterior, o $G$-módulo regular $\mathbb{C} G$ é completamente redutível. Na verdade vale que:

Teorema 3.2.2 ([JL], pg.91) Seja $\mathbb{C} G=U_{1} \oplus \cdots \oplus U_{r}$, onde cada $U_{i}$ é um $G$ submódulo irredutivel, então todo $G$-módulo irredutivel $U$ é isomorfo a $U_{i}$ para algum $i$.

Teorema 3.2.3 ([JL], pg.100) Seja $\mathbb{C} G=U_{1} \oplus \cdots \oplus U_{r}$, onde cada $U_{i}$ é um $G$ submódulo irredutivel e $U$ um $G$-módulo irredutível. Então o número de $U_{i}^{\prime} s$ distintos tais que $U \simeq U_{i}$ é igual a dimensão de $U$.

Estabelecemos portanto o vínculo entre as representações irredutíveis do grupo finito $G$ e a decomposição de $\mathbb{C} G$. O próximo resultado vai nos possibilitar conectar estudo das representações de $S_{n}$ aos diagramas de Young.

Teorema 3.2.4 [Cohn] O número de representações irredutíveis não equivalentes é igual ao número de classes de conjugação de $G$.

\subsubsection{Representações de $S_{n}$}

Veremos agora a relação entre a álgebra de grupo de $S_{n}, \mathbb{C} S_{n}$ e os diagramas de Young. Sabemos que cada permutação $p \in S_{n}$ pode ser escrita como produto de ciclos disjuntos. Como esses ciclos comutam, podemos rearranjá-los pela ordem de tamanho de forma decrescente.

Suponha $p=c_{1} c_{2} \ldots c_{k}$, onde os $c_{i}{ }^{\prime} s$ são ciclos disjuntos e $\lambda_{1}, \lambda_{2}, \ldots, \lambda_{k}$ seus respectivos tamanhos. Logo $\lambda_{1}+\lambda_{2}+\cdots+\lambda_{k}=n$ e $\lambda_{1} \geq \lambda_{2} \geq \cdots \geq \lambda_{k}$. Logo, à cada estrutura de ciclos de uma permutação podemos associar uma partição de $n$ e portanto um diagrama de Young. 
Quando duas permutações $p$ e $q$ possuem a mesma estrutura de ciclos é fácil definir um elemento $f \in S_{n}$ tal que $f^{-1} p f=q$. Por outro lado, se $p$ e $q$ são permutações conjugadas, então possuem a mesma estrutura de ciclos (basta observar que se $c$ é um $k$-ciclo, $f^{-1} c f$ também é um $k$-ciclo). Sendo assim temos que:

Proposição 3.2.2 Existe uma bijeção entre as classes de conjugação em $S_{n}$ e os diagramas de Young de $n$-células.

Reunindo os resultados anteriores, chegamos à seguinte conclusão :

Cada diagrama de Young corresponde a uma representação irredutível de $S_{n}$ e portanto a um fator da decomposição de $\mathbb{C} S_{n}$.

Vamos mostrar agora a relação explicíta entre um diagrama de Young e a representação irredutível associada.

Definições 3.2.3 Seja $\lambda$ um diagrama de Young e $D_{\lambda}$ um tableau associado a $\lambda$. Considere os seguintes subgrupos de $S_{n}$ :

$$
\begin{gathered}
P_{D_{\lambda}}=\left\{p \in S_{n} / \text { as linhas de } D_{\lambda} \text { são invariantes sob } p\right\} \\
Q_{D_{\lambda}}=\left\{q \in S_{n} / \text { as colunas de } D_{\lambda} \text { são invariantes sob } q\right\}
\end{gathered}
$$

Por exemplo, quando $D_{\lambda}$ é o tableau:

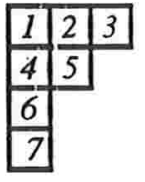

$P_{D_{\lambda}}$ é gerado pelas transposições $\{(12),(23),(45)\}$ e $Q_{D_{\lambda}}$ gerado pelas transposições $\{(14),(46),(67),(25)\}$.

Definições 3.2.4 Sejam $A_{D_{\lambda}}=\sum_{p \in P_{D_{\lambda}}} p \quad$ e $\quad B_{D_{\lambda}}=\sum_{q \in Q_{D_{\lambda}}}(-1)^{l(q)} q$ onde $l(q)$ é o tamanho da permutação $q$. O simetrizador de Young $C_{D_{\lambda}}$ é o elemento de $\mathbb{C} S_{n}$ definido por $C_{D_{\lambda}}=A_{D_{\lambda}} B_{D_{\lambda}}$. 
Obs: Estamos considerando a multiplicação em $S_{n}, p . q$, efetuando-se primeiro a permutação $p$ e depois a permutação $q$.

Proposição 3.2.3 Sejam $D^{\prime}$ e $D$ tableaux associados ao mesmo diagrama $\lambda$ de $n$ células. Entẫo:

$$
C_{D}=\tau C_{D^{\prime}} \tau^{-1}
$$

onde $\tau \in S_{n}$.

\section{Demonstração:}

Sejam $D(i, j)$ e $D^{\prime}(i, j)$ os números associados à célula $\lambda_{i, j}$ em $D$ e $D^{\prime}$ respectivamente. Seja $\tau \in S_{n}$ tal que $\tau(D(i, j))=D^{\prime}(i, j)$. Note que $\tau$ leva as linhas de $D$ nas linhas de $D^{\prime}$ e as colunas de $D$ nas colunas de $D^{\prime}$.

Seja $p^{\prime} \in P_{D^{\prime}}$. Então $\tau p^{\prime} \tau^{-1}$ é uma permutação que preserva as linhas de $D$, isto é, pertence a $P_{D}$. Por outro lado, para todo $p \in P_{D}, \tau\left(\tau^{-1} p \tau\right) \tau^{-1}=p$, onde $\tau^{-1} p \tau \in P_{D^{\prime}}$. Logo:

$$
P_{D}=\left\{\tau p^{\prime} \tau^{-1}: p^{\prime} \in P_{D^{\prime}}\right\}
$$

Sendo assim, $A_{D}=\tau A_{D^{\prime}} \tau^{-1}$. Analogamente, temos que $B_{D}=\tau B_{D^{\prime}} \tau^{-1}$. Portanto:

$$
C_{D}=A_{D} B_{D}=\tau A_{D^{\prime}} B_{D^{\prime}} \tau^{-1}=\tau C_{D^{\prime}} \tau^{-1}
$$

Teorema 3.2.5 ([FH] , pg.52)

(i) $C_{D_{\lambda}}^{2}=a_{D_{\lambda}} C_{D_{\lambda}}$, onde $a_{D_{\lambda}} \in \mathbb{C} \backslash\{0\}$.

(ii) $V_{D_{\lambda}}=\mathbb{C} S_{n} C_{D_{\lambda}}$ é uma representação irredutível de $S_{n}$. Toda representação irredutivel de $S_{n}$ pode ser obtida desse modo para uma única partição $\mu$.

(iii) $a_{D_{\lambda}}=\frac{n !}{\operatorname{dim} V_{\lambda}}$.

(iv) $V_{D_{\lambda}}$ e $V_{D_{\mu}}$ são isomorfas se e somente se $\lambda=\mu$.

(v) $\mathbb{C} S_{n}=\underset{|\lambda|=n}{\bigoplus} \mathbb{C} S_{n} C_{D_{\lambda}} \mathbb{C} S_{n}$. 
Proposição 3.2.4 [FH] A dimensão do sub-módulo $\mathbb{C} S_{n} C_{\lambda}$ é o número de tableaux padrões associados a $\lambda$, isto é:

$$
\operatorname{dim} \mathbb{C} S_{n} C_{\lambda}=d_{\lambda}
$$

onde $d_{\lambda}=\frac{n !}{\prod h l\left(\lambda_{i, j}\right)}($ verProp. 3.1.1).

Corolário 3.2.1 Seja $a_{D_{\lambda}}$ tal que:

$$
C_{D_{\lambda}}^{2}=a_{D_{\lambda}} C_{D_{\lambda}}
$$

onde $C_{D_{\lambda}}$ é o simetrizador de Young para algum tableau $D_{\lambda}$. Então:

$$
a_{D_{\lambda}}=\prod h l\left(\lambda_{i, j}\right) .
$$

Definição 3.2.2 Seja $\lambda$ um diagrama de Young de $n$ células e considere $T(\lambda)$ o tableau onde as células são numeradas de 1 a $n$ sucessivamente ao longo das linhas de $\lambda$.

Pelos resultados anteriores, a decomposição $\mathbb{C} S_{n}=\bigoplus_{|\lambda|=n} \mathbb{C} S_{n} C_{D_{\lambda}} \mathbb{C} S_{n}$ e o valor do escalar associado aos simetrizadores de um mesmo diagrama $\lambda$, não dependem do tableau escolhido. Vamos portanto considerar $D_{\lambda}$ o tableau padrão $T(\lambda)$ definido acima. Denotaremos, nesse caso, $C_{T(\lambda)}$ por $C_{\lambda}$, bem como $a_{T(\lambda)}$ por $a_{\lambda}$. Nessa notação temos que:

$$
\mathbb{C} S_{n}=\bigoplus_{|\lambda|=n} \mathbb{C} S_{n} C_{\lambda} \mathbb{C} S_{n}
$$

\subsection{Construção dos idempotentes}

Motivados pelo fato de que $H_{n}$ é uma espécie de deformação de $\mathbb{C} S_{n}$, vamos decompor $H_{n}$ em termos de idempotentes usando os diagramas de Young. Para isso, precisamos de mais alguns resultados e definições sobre esses diagramas.

Definição 3.3.1 Seja $\pi_{\lambda} \in S_{n}$ tal que $\pi_{\lambda}(i)=j$ onde a célula numerada com $i$ em $T(\lambda)$ é levada pela transposição na célula numerada com $j$ em $T\left(\lambda^{v}\right)$. Observe que $\pi_{\lambda}^{-1}=\pi_{\lambda^{v}}$. 


\section{Exemplo:}

$$
\begin{aligned}
& \begin{array}{|l|l|l|l|}
\hline 1 & 2 & 3 & 4 \\
\hline
\end{array} \\
& \begin{array}{lll}
5 & 6 \\
\hline
\end{array} \\
& 7 \\
& \nu=(4,2,1), \pi_{\nu}=(247365)
\end{aligned}
$$

Seja $R(\lambda)$ o subgrupo de $S_{n}$ que mantém as linhas de $T(\lambda)$ invariantes. É fácil ver que $R(\lambda)$ é gerado por transposições elementares do tipo $(i i+1)$. No exemplo acima temos $R(\nu)=<(12),(13),(34),(56)>$.

Sejam $\lambda$ e $\mu$ diagramas de Young tais que $|\lambda|=|\mu|=n$. Dizemos que $\pi \in S_{n}$ separa $\lambda$ de $\mu$ se nenhum par de números na mesma linha de $T(\lambda)$ é levado por $\pi$ na mesma linha de $T(\mu)$. Como exemplo, temos que $\pi_{\lambda}$ separa $\lambda$ e $\lambda^{v}$.

Caso nenhuma permutação $\pi$ separe $\lambda$ e $\mu$ dizemos que são inseparáveis. Observe que se $\pi \in S_{n}$ separa $\lambda$ e $\mu$, então $\rho \pi \tau$ também o faz, quando $\tau \in R(\mu)$ e $\rho \in R(\lambda)$.

Vamos colocar uma ordem no conjunto dos diagramas de Young, a ordem lexicográfica, da seguinte maneira:

$$
\lambda>\mu \Leftrightarrow \exists t \operatorname{com} \lambda_{t}>\mu_{t} \text { e } \lambda_{i}=\mu_{i} \text { para } i<t
$$

onde $\lambda_{i}$ é o número de células da i-ésima linha de $\lambda$.

Obs: Para podermos efetuar essa comparação quando o número de linhas de $\lambda$ for menor que o de $\mu$ (por exemplo), completamos as linhas restantes do último colocando $\mu_{i}=0$.

Lema 3.3.1 Sejam $\lambda$ e $\mu$ dois diagramas de Young tais que $|\lambda|=|\mu|=n$. Se $\lambda>\mu$ então $\lambda$ e $\mu^{v}$ são inseparáveis.

\section{Demonstração:}


Vamos demonstrar usando indução no número de células. Para $n=1, \lambda=\mu \mathrm{e}$ portanto nada há a fazer. Da mesma forma, para $n=2$, temos $\lambda=\mu^{v}$, e portanto claramente inseparáveis. Assumimos o resultado para $\nu$ tal que $|\nu|\langle n$ onde $n>2$.

Sejam $\lambda=\left(\lambda_{1}, \lambda_{2}, \ldots, \lambda_{k}\right)$ e $\mu=\left(\mu_{1}, \mu_{2}, \ldots, \mu_{m}\right)$ diagramas de Young com mais de $n$ células. Observamos que $\mu_{1}$ é o número de células da primeira coluna de $\mu^{v}$.

Se $\lambda_{1}>\mu_{1}$, o número de células na primeira linha de $\lambda$ é maior que o número de linhas de $\mu^{v}$ e portanto, qualquer permutação deve levar pelo menos dois números da primeira linha de $\lambda$ em uma mesma linha de $\mu^{v}$. Logo nesse caso, os diagramas são inseparáveis.

Se $\lambda_{1}=\mu_{1}$, considere os diagramas $r(\lambda)$ e $r(\mu)$ obtidos removendo-se a primeira linha de $\lambda$ e $\mu$ respectivamente. Sendo assim, $|r(\lambda)|=\left|r(\mu)^{v}\right|\langle n$ e como $\lambda\rangle \mu$ temos $r(\lambda)>r(\mu)$. Pela hipótese de indução, $r(\lambda)$ e $r(\mu)^{v}$ são inseparáveis.

Basta mostrarmos portanto que se $\lambda$ e $\mu^{v}$ fossem separáveis, então $r(\lambda)$ e $r(\mu)^{v}$ também o seriam.

De fato, supondo $\lambda$ e $\mu^{v}$ separáveis, considere $\pi$ uma permutação que separa esse diagramas. Como $\lambda_{1}=\mu_{1}$, o número de células na primeira coluna de $\lambda$ é igual ao número de linhas de $\mu^{v}$. Logo $\pi$ deve mandar exatamente cada célula da primeira linha de $\lambda$ para cada linha de $\mu^{v}$.

Seja $s \in R\left(\mu^{v}\right)$ a permutação tal que $\pi s$ manda cada célula da primeira linha de $\lambda$ para a primeira célula de cada linha de $\mu^{v}$, isto é manda a primeira linha de $\lambda$ exatamente na primeira coluna de $\mu^{v}$.

Portanto, $\pi s \in S_{n}$ separa $\lambda$ e $\mu^{v}$. Restringindo-a a $r(\lambda)$, temos que a imagem também se restringe a $r(\mu)^{v}$ e consequentemente separa $r(\lambda)$ e $r(\mu)^{v}$.

Corolário 3.3.1 Dados $\lambda$ e $\mu$ tais que $|\lambda|=|\mu|=n$, uma das afirmações abaixo é verdadeira:

(1) $\lambda$ e $\mu^{v}$ são inseparáveis;

(2) $\lambda^{v}$ e $\mu$ são inseparáveis;

(3) $\lambda=\mu$. 
Vamos agora construir os elementos básicos que serão usados na construção dos idempotentes.

Definição 3.3.2 Seja $\boldsymbol{E}_{n}=\sum_{\pi \in S_{n}} \boldsymbol{\omega}_{\pi}$ onde $\omega_{\pi}$ é a trança de permutação positiva associada à permutação $\pi$ (ver cap.1).

Teorema 3.3.1 Para cada $i \in \mathbb{N}$ tal que $1 \leq i \leq n-1$, podemos fatorar $E_{n}$ em $H_{n}$ como:

$$
E_{n}=E_{n}^{(i)}\left(\sigma_{i}+1\right)=\left(\sigma_{i}+1\right) E_{n}^{(i)}
$$

onde $E_{n}^{(i)}=\sum_{\pi(i)<\pi(i+1)} \omega_{\pi}$.

\section{Demonstração:}

Conseguimos escrever $S_{n}$ como a união disjunta de dois conjuntos:

$$
\begin{gathered}
A_{i}=\left\{\pi \in S_{n} / \pi(i)<\pi(i+1)\right\} \\
e \\
B_{i}=\left\{\pi \in S_{n} / \pi(i+1)<\pi(i)\right\} .
\end{gathered}
$$

Observe que existe uma bijeção entre esses dois conjuntos tomando para cada $\pi \in A_{i}$ o elemento $\pi^{\prime}=(i i+1) \pi \in B_{i}$. Além disso, temos que $\sigma_{i} \omega_{\pi}$ só possui cruzamentos positivos e a informação $\pi(i)<\pi(i+1)$ nos garante que a i-ésima e a (i+1)-ésima linhas só se cruzam uma vez.

Portanto, pelo Teorema 2.3.1 $\sigma_{i} \omega_{\pi}$ é a trança de permutação positiva associada à $\pi^{\prime}$, isto é $\omega_{\pi^{\prime}}=\sigma_{i} \omega_{\pi}$.

Sendo assim,

$$
E_{n}=\sum_{\pi \in S_{n}} \omega_{\pi}=\sum_{\pi \in A_{i}} \omega_{\pi}+\sum_{\pi^{\prime} \in B_{i}} \omega_{\pi^{\prime}}
$$

E devido à bijeção entre $A_{i}$ e $B_{i}$ temos que:

$$
E_{n}=\sum_{\pi \in A_{i}} \omega_{\pi}+\sum_{\pi \in A_{i}} \sigma_{i} \omega_{\pi}=\left(1+\sigma_{i}\right) E_{n}^{(i)} .
$$


Afirmamos que

$$
\sigma_{i} \omega_{\pi}=\omega_{\pi} \sigma_{i}
$$

para todo $\pi \in A_{i}$. De fato, como $\omega_{\pi}$ é trança de permutação positiva e $\pi(i)<\pi(i+1)$, toda $j$-linha cruza acima ou abaixo de ambas as linhas $i$ e $i+1$. Logo, podemos "passar para baixo" de $\omega_{\pi}$ o cruzamento realizado por $\sigma_{i}$ nessas linhas fazendo uma isotopia ambiente no plano formado por elas, ou em termos de diagramas, efetuando uma sequência de movimentos $R I I$ e $R I I I$. Logo, a outra igualdade também é válida.

Dado um escalar $\gamma \in \Lambda$, podemos substituir $\sigma_{i}$ por $\gamma \sigma_{i}$ em $\omega_{\pi}$ que é na verdade um monômio nos $\sigma_{i}^{\prime} s$. Logo:

$$
\omega_{\pi}\left(\gamma \sigma_{1}, \ldots, \gamma \sigma_{n-1}\right)=\gamma^{l(\pi)} \omega_{\pi}\left(\sigma_{1}, \ldots, \sigma_{n-1}\right)
$$

onde $l(\pi)$ é tanto o tamanho da permutação $\pi$ como o número de auto-enlaçamento de $\omega_{\pi}$.

Definições 3.3.1 Lembremos (seção 1.5) que as tranças elementares geradoras de $H_{n}$ satisfazem uma relação quadrática:

$$
\left(\sigma_{i}-a\right)\left(\sigma_{i}-b\right)=0
$$

onde $a=-x s^{-1}$ e $b=x s$.

Definimos então:

$$
\begin{gathered}
a_{n}=E_{n}\left(-a^{-1} \sigma_{1}, \ldots,-a^{-1} \sigma_{n-1}\right) \\
b_{n}=E_{n}\left(-b^{-1} \sigma_{1}, \ldots,-b^{-1} \sigma_{n-1}\right)
\end{gathered}
$$

e portanto

$$
\begin{aligned}
& a_{n}=\sum_{\pi \in S_{n}}(-a)^{-l(\pi)} \omega_{\pi} \\
& b_{n}=\sum_{\pi \in S_{n}}(-b)^{-l(\pi)} \omega_{\pi} .
\end{aligned}
$$


Corolário 3.3.2 Para todo $i \in \mathbb{N}, 1 \leq i \leq n-1$, $a_{n}$ e $b_{n}$ fatoram-se em $H_{n}$ da seguinte maneira:

$$
\begin{aligned}
a_{n} & =\left(\sigma_{i}-a\right) a_{n}^{(i)}=a_{n}^{(i)}\left(\sigma_{i}-a\right) \\
b_{n} & =\left(\sigma_{i}-b\right) b_{n}^{(i)}=b_{n}^{(i)}\left(\sigma_{i}-b\right)
\end{aligned}
$$

\section{Demonstração:}

Basta aplicar o teorema anterior sustituindo $\sigma_{i}$ por $-a^{-1} \sigma_{i} \mathrm{e}-b^{-1} \sigma_{i}$ respectivamente.

Teorema 3.3.2 Sejam $\phi_{a}$ e $\phi_{b}$ homomorfismos lineares definidos por $\phi_{a}\left(\sigma_{i}\right)=a e$ $\phi_{b}\left(\sigma_{i}\right)=b$ para $i=1, \ldots, n-1$. Então, para qualquer $h \in H_{n}$ :

$$
\begin{gathered}
a_{n} h=h a_{n}=\phi_{b}(h) a_{n} \\
b_{n} h=h b_{n}=\phi_{a}(h) b_{n}
\end{gathered}
$$

\section{Demonstração:}

Como $\phi_{a}$ e $\phi_{b}$ são homomorfismos lineares basta mostrar as igualdades para as tranças elementares $\sigma_{i}$, isto é, temos que mostrar que:

$$
\begin{gathered}
a_{n} \sigma_{i}=b a_{n}=\sigma_{i} a_{n} \\
b_{n} \sigma_{i}=a b_{n}=\sigma_{i} b_{n}
\end{gathered}
$$

Usando o Colorário 3.3.2, temos:

$$
a_{n}=a_{n}^{(i)}\left(\sigma_{i}-b\right)
$$

Logo:

$$
a_{n}\left(\sigma_{i}-b\right)=a_{n}^{(i)}\left(\sigma_{i}-a\right)\left(\sigma_{i}-b\right)=0
$$


E portanto $a_{n} \sigma_{i}=b a_{n}$. Analogamente, $b_{n}\left(\sigma_{i}-a\right)=0 \Rightarrow b_{n} \sigma_{i}=a b_{n}$.

Usando as fatorações $a_{n}=\left(\sigma_{i}-a\right) a_{n}^{(i)}$ e $b_{n}=\left(\sigma_{i}-b\right) a_{n}^{(i)}$ provamos as igualdades restantes.

Obs: Note que podemos incluir $H_{k}$ em $H_{n}$, quando $k \leq n$, usando a operação $\otimes$ definida na seção 2.3. Logo o Lema anterior nos diz que, em particular, quando $k \leq n$ temos:

$$
\begin{gathered}
a_{k} a_{n}=a_{n} a_{k}=\phi_{b}\left(a_{k}\right) a_{n} \\
e \\
b_{k} b_{n}=b_{n} b_{k}=\phi_{a}\left(b_{k}\right) b_{n} .
\end{gathered}
$$

Definição 3.3.3 Seja $\lambda=\left(\lambda_{1}, \lambda_{2}, \ldots, \lambda_{k}\right)$ um diagrama de Young com $|\lambda|=n$. Lembrando que $\bar{\omega}_{\tau}$ é a trança de permutação negativa associada à permutação $\tau$, seja:

$$
e_{\lambda}=E_{\lambda}(a) \omega_{\pi_{\lambda}} E_{\lambda^{v}}(b) \bar{\omega}_{\pi_{\lambda^{v}}} \in H_{n}
$$

onde $E_{\lambda}(a)=a_{\lambda_{1}} \otimes a_{\lambda_{2}} \otimes \cdots \otimes a_{\lambda_{k}}$ e $E_{\lambda}(b)=b_{\lambda_{1}^{v}} \otimes b_{\lambda_{2}^{v}} \otimes \cdots \otimes b_{\lambda_{j}^{v}}$.

Obs: Lembramos que $\pi_{\lambda}^{-1}=\pi_{\lambda^{v}}$ e que portanto $\bar{\omega}_{\pi_{\lambda^{v}}}$ é o inverso de $\omega_{\pi_{\lambda}}$.

Exemplos:

1)

$$
\begin{aligned}
& a_{2}=\|+x^{-1} s \\
& b_{2}=\|-x^{-1} s^{-1}
\end{aligned}
$$


2) Para $\nu=(3,2,1,1)$ temos $e_{\nu}$ igual a:

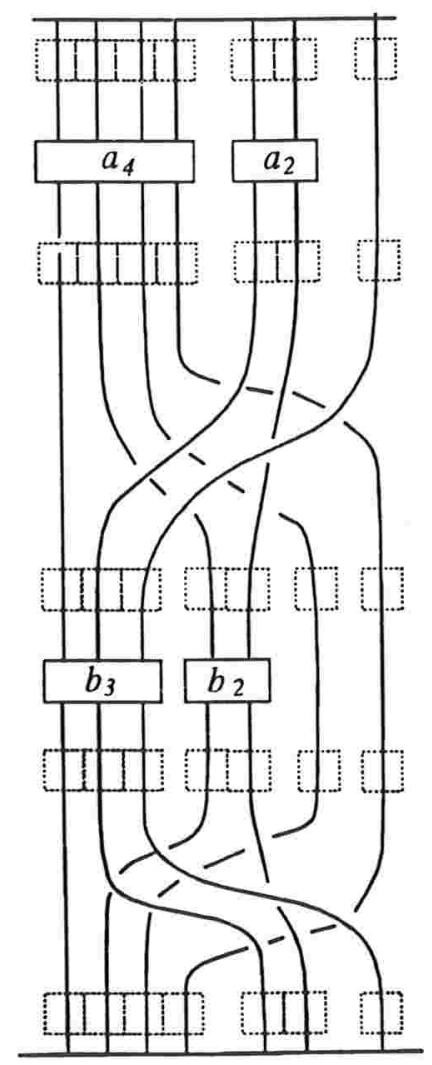

Faremos a partir de agora uma série de Lemas de natureza técnica a fim de provarmos um dos resultados importantes desse capítulo: os elementos $e_{\lambda}$ são quaseidempotentes (isto é, são tais que $e_{\lambda}^{2}=\alpha_{\lambda} e_{\lambda}$, para algum $\alpha_{\lambda} \in \Lambda$ ) e são ortogonais (isto é, se $\lambda \neq \mu$, então $e_{\lambda} e_{\mu}=0$ ). Antes, porém, vamos estabelecer uma convenção a fim de evitarmos ambiguidades devido à linguagem envolvida nesses lemas que misturam "linhas de tranças" com "linhas de diagramas de Young".

Convenção: Sejam $\omega$ uma n-trança e $\lambda$ um diagrama de Young, tal que $|\lambda|=n$. Podemos agrupar as linhas de $\omega$ de maneira natural, fazendo com que correspondam às células de $T(\lambda)$ associando a $i$-linha de $\omega$ com a célula numerada com $i$ no tableau $T(\lambda)$. Diremos nesse caso que essa $i$-linha é uma $\lambda_{j}$-linha, onde $j$ significa que a célula numerada com $i$ em $T(\lambda)$ encontra-se na $j$-ésima linha de $\lambda$. 
Lema 3.3.2 Seja $\lambda=\left(\lambda_{1}, \ldots, \lambda_{k}\right)$ um diagrama de Young. Seja $\boldsymbol{H}(\boldsymbol{\lambda}) \subseteq \boldsymbol{H}_{|\lambda|}$ a sub-álgebra gerada por $\left\{\omega_{\rho} / \rho \in R(\lambda)\right\}$. Então $E_{\lambda}(a), E_{\lambda}(b) \in H(\lambda)$ e valem as seguintes igualdades para todo $h \in H(\lambda)$ :

$$
\begin{gathered}
E_{\lambda}(a) h=h E_{\lambda}(a)=\phi_{b}(h) E_{\lambda}(a) \\
e \\
E_{\lambda}(b) h=h E_{\lambda}(b)=\phi_{a}(h) E_{\lambda}(b)
\end{gathered}
$$

\section{Demonstração:}

Primeiramente observamos que todo elemento $h \in H(\lambda)$ só cruza linhas associadas à mesma linha de $\lambda$ e portanto pode ser decomposto da seguinte forma:

$$
h=h_{\lambda_{1}} \otimes h_{\lambda_{2}} \otimes \cdots \otimes h_{\lambda_{k}}
$$

onde cada $h_{\lambda_{i}}$ só age nas $\lambda_{i}$-linhas. Logo, podemos escrever $h$ na forma:

$$
h=\left(h_{\lambda_{1}} \otimes 1^{\otimes \lambda_{2}} \otimes \cdots \otimes 1^{\otimes \lambda_{k}}\right) \cdot\left(1^{\otimes \lambda_{1}} \otimes h_{\lambda_{2}} \otimes \cdots \otimes 1^{\otimes \lambda_{k}}\right) \ldots\left(1^{\otimes \lambda_{1}} \otimes \cdots \otimes h_{\lambda_{k}}\right)
$$

E como $\phi_{b}$ é homomorfismo linear vale $\phi_{b}(h)=\phi_{b}\left(h_{\lambda_{1}}\right) \phi_{b}\left(h_{\lambda_{2}}\right) \ldots \phi_{b}\left(h_{\lambda_{k}}\right)$, e portanto, pelo Teorema 3.3.2, temos que:

$$
E_{\lambda}(a) h=\phi_{b}(h) E_{\lambda}(a) .
$$

As outras igualdades saem de forma completamente análoga.

Lema 3.3.3 Se $\pi^{\prime}=\rho \pi$, onde $\rho=(i j)$, então existe uma trança $h$ tal que $\omega_{\pi^{\prime}}=$ $h \omega_{\pi^{\prime}}$. Analogamente, se $\pi^{\prime}=\pi \rho$, então existe uma trança $h^{\prime}$ tal que $\omega_{\pi^{\prime}}=\omega_{\pi} h^{\prime}$.

\section{Demonstração:}

Seja $\rho=(i j)$, com $i<j$ e considere $\pi^{\prime}=\rho \pi$. Queremos uma trança $h$ cuja permutação associada seja $\rho$ e tal que $h \omega_{\pi}$ seja uma trança de permutação positiva. Pela Prop. 1.4.2 esse elemento tem que ser $\omega_{\pi^{\prime}}$.

Podemos construir $h$ de modo que:

(1) as linhas de $h$ são todas triviais, excetuando-se as linhas $i$ e $j$. 
(2) as linhas $i$ e $j$ cruzam as linhas intermediárias apenas uma vez.

(3) as linhas $i$ e $j$ não cruzam as demais linhas.

(4) o sinal de cada cruzamento é definido pela regra:

(i) Considere o cruzamento efetuado por duas linhas em $h$.

(ii) Verifique se essa linhas se cruzam em $\omega_{\pi}$.

(iii) Caso haja cruzamento em $\omega_{\pi}$ defina o cruzamento em $h$ como negativo, caso contrário, defina como positivo.

Observamos que a operação além de ser realizada em um número finito de passos, está bem definida, pois em $\omega_{\pi}$ cada par de linhas se cruzam no máximo uma vez.

Com $h$ assim definido, cada cruzamento negativo nesse elemento "anula" um cruzamento realizado em $\omega_{\pi}$ de tal modo que, efetuada uma isotopia regular, as mesmas linhas no produto $h \omega_{\pi}$ não se cruzam. Logo, $h \omega_{\pi}$ é uma trança de permutação positiva tendo $\rho \pi=\pi^{\prime}$ como permutação associada, como queríamos, o outro caso obriamente análogo.

Lema 3.3.4 Sejam $\lambda$ e $\mu$ diagramas de Young tais que $|\lambda|=|\mu|=n$ e $\lambda \neq \mu$. Se $\pi \in S_{n}$ não separa $\lambda$ e $\mu$ então:

$$
\begin{aligned}
& E_{\lambda}(a) \omega_{\pi} E_{\mu}(b)=0 \\
& E_{\mu}(b) \omega_{\pi} E_{\lambda}(a)=0
\end{aligned}
$$

\section{Demonstração:}

Vamos mostrar que $E_{\lambda}(a) \omega_{\pi} E_{\mu}(b)=0$ sendo análoga a prova da outra afirmação. Seja $\pi$ que não separa $\lambda$ e $\mu$. Nesse caso devem existir duas células da mesma linha de $\lambda$, digamos a l-ésima, que são mandadas na mesma linha de $\mu$, digamos a p-ésima.

Suponha que as duas células de $\lambda$ citadas sejam adjacentes e sejam mandadas em células também adjacentes. Caso não sejam, considere $\rho$ e $\sigma$ duas transposições tais que $\pi^{\prime}=\rho \pi \sigma$ manda as células $i$ e $i+1$ da l-ésima linha de $\lambda$ para as células $j$, $j+1$ da p-ésima linha de $\mu$ respectivamente. Pelo Lema 3.3.3, temos que

$$
\omega_{\pi}=h \omega_{\pi^{\prime}} h^{\prime}
$$


onde $h$ e $h^{\prime}$ são $n$-tranças. Logo pelo Lema anterior:

$$
E_{\lambda}(a) \omega_{\pi} E_{\mu}(b)=\phi_{b}(h) \phi_{a}\left(h^{\prime}\right) E_{\lambda}(a) \omega_{\pi^{\prime}} E_{\mu}(b)
$$

Temos que $\phi_{b}(h)$ e $\phi_{a}(h)$ são não nulos, pois $h$ e $h^{\prime}$ são, nesse caso, monômios nas tranças geradoras e portanto esses escalares são potências de $b$ e de $a$ respectivamente. Sendo assim, podemos assumir que duas $\lambda_{l}$-linhas $i$ e $i+1$, são mandadas nas duas $\mu_{p}$-linhas $j$ e $j+1$, respectivamente.

Como as linhas $i$ e $i+1$ terminam em pontos adjacentes e como estamos trabalhando com uma trança de permutação positiva, todas as demais linhas passam por baixo ou por cima de ambas. Logo, usando os movimentos RII e RIII, conseguimos "passar o cruzamento para baixo" de $\omega_{\pi}$. Isto é, vale que:

$$
\sigma_{i} \omega_{\pi}=\omega_{\pi} \sigma_{j}
$$

Por outro lado, pelo Corolário 3.3.2, temos que:

$$
\begin{aligned}
& a_{\lambda_{l}}=a_{\lambda_{l}}^{(i)}\left(\sigma_{i}-a\right) \\
& b_{\mu_{p}}=\left(\sigma_{j}-b\right) b_{\mu_{p}}^{(j)}
\end{aligned}
$$

Observamos que:

$$
E_{\lambda}(a) \omega_{\pi}=\left(a_{\lambda_{1}} \otimes \cdots \otimes 1^{\otimes\left|\lambda_{l}\right|} \otimes \cdots a_{\lambda_{k}}\right)\left(1^{\otimes l_{1}} \otimes a_{\lambda_{l}} \otimes 1^{\otimes l_{2}}\right) \omega_{\pi}=\widetilde{E_{\lambda}(a)}\left(\sigma_{i}-a\right) \omega_{\pi}
$$

onde $l_{1}+\lambda_{l}+l_{2}=|\lambda|$.

E de forma análoga, concluimos que:

$$
\omega_{\pi} E_{\mu}(b)=\omega_{\pi}\left(\sigma_{j}-b\right) \widetilde{E_{\mu}(b)} .
$$

Ou seja:

$$
E_{\lambda}(a) \omega_{\pi} E_{\mu}(b)=\widetilde{E_{\lambda}(a)}\left(\sigma_{i}-a\right) \omega_{\pi}\left(\sigma_{j}-b\right) \widetilde{E_{\mu}(b)}
$$

Pela equação 3.2 temos:

$$
\left(\sigma_{i}-a\right) \omega_{\pi}=\omega_{\pi}\left(\sigma_{j}-a\right)
$$


E portanto:

$$
\left(\sigma_{i}-a\right) \omega_{\pi}\left(\sigma_{j}-b\right)=\omega_{\pi}\left(\sigma_{j}-a\right)\left(\sigma_{j}-b\right)
$$

Por outro lado, temos que $\left(\sigma_{j}-a\right)\left(\sigma_{j}-b\right)=0$ em $H_{n}$ e portanto:

$$
E_{\lambda}(a) \omega_{\pi} E_{\mu}(b)=0
$$

Corolário 3.3.3 Sejam $\lambda$ e $\mu$ diagramas de Young tais que $|\lambda|=|\mu|=n$ e $\lambda \neq \mu$. Se $\lambda$ e $\mu$ são inseparáveis então:

$$
\begin{aligned}
& E_{\lambda}(a) H_{n} E_{\mu}(b)=0 \\
& E_{\mu}(b) H_{n} E_{\lambda}(a)=0
\end{aligned}
$$

\section{Demonstração:}

Lembramos que $H_{n}$ é gerada como $\Lambda$-módulo pelas tranças de permutação positiva $\left\{\omega_{\pi} / \pi \in S_{n}\right\}$ (Teorema 2.3.1). Logo basta aplicar o Lema 3.3.4.

Lema 3.3.5 Seja $\lambda=\left(\lambda_{1}, \ldots, \lambda_{k}\right)$ um diagrama de Young tal que $|\lambda|=n$. Seja $\tau \in S_{n}$ que separa $\lambda$ e $\lambda^{v}$. Então existem $\rho_{1} \in R(\lambda)$ e $\rho_{2} \in R\left(\lambda^{v}\right)$ tais que $\rho_{1} \tau \rho_{2}=\pi_{\lambda}$.

\section{Demonstração:}

Observe primeiramente que $\lambda^{v}$ possui $\lambda_{1}$ linhas. Como $\tau$ separa $\lambda$ e $\lambda^{v}$, cada célula da primeira linha de $\lambda$ tem que ser levada exatamente em cada linha de $\lambda^{v}$, restando portanto nesse diagrama $\lambda_{2}$ linhas com mais de uma célula.

Logo a segunda linhas de $\lambda$ tem que ser levada nas $\lambda_{2}$ primeiras linhas de $\lambda^{v}$ (novamente usando que $\tau$ separa os dois diagramas). Continuando o processo, concluímos que cada célula da $j$-ésima linha de $\lambda$ é mandada obrigatoriamente por $\tau$ para uma célula de cada uma das primeiras $\lambda_{j}$ linhas de $\lambda^{v}$.

Seja $\rho_{1, j} \in R(\lambda)$ a permutação que reordena as células da j-ésima linha de $\lambda$, de modo que a célula $\lambda_{j, i}$ seja levada na i-ésima linha de $\lambda^{v}$ por $\rho_{1, j}$.

Considere $\rho_{1}=\prod_{j=1}^{k} \rho_{1, j}$. Note que $\rho_{1, j}$ e $\rho_{1, j^{\prime}}$ comutam quando $j \neq j^{\prime}$ e portanto $\rho_{1} \in R(\lambda)$. 
De forma análoga, considere $\rho_{2, j} \in R\left(\lambda^{v}\right)$ a permutação que reordena as células das $j$-ésima linha de $\lambda^{v}$ de tal modo que a célula $\lambda_{j, i}^{v}$ seja a imagem de uma célula da $i$-ésima linha de $\lambda$ sob $\rho_{1} \tau \rho_{2, j}$. Novamente temos que $\rho_{2}=\prod_{j=1}^{\lambda_{1}} \rho_{2, j} \in R\left(\lambda^{v}\right)$. Sendo assim, temos uma permutação $\rho_{1} \tau \rho_{2}$, onde $\rho_{1} \in R(\lambda)$ e $\rho_{2} \in R\left(\lambda^{v}\right)$, a qual manda a célula $\lambda_{i, j}$ na célula $\lambda_{j, i}^{v}$, isto é, $\rho_{1} \tau \rho_{2}=\pi_{\lambda}$.

Corolário 3.3.4 Seja $\lambda$ um diagrama de Young. Seja $\tau$ uma permutação que separa $\lambda e \lambda^{v}$. Então:

$$
\omega_{\tau}=\omega_{\rho_{1}^{-1}} \omega_{\pi_{\lambda}} \omega_{\rho_{2}^{-1}}
$$

onde $\rho_{1}^{-1} \in R(\lambda)$ e $\rho_{2}^{-1} \in R\left(\lambda^{v}\right)$.

\section{Demonstração:}

Do Lema anterior, temos que $\tau=\rho_{1}^{-1} \pi_{\lambda} \rho_{2}^{-1}$, onde $\rho_{1}^{-1} \in R(\lambda)$ e $\rho_{2}^{-1} \in R\left(\lambda^{v}\right)$. Vamos mostrar que $\omega_{\tau}=\omega_{\rho_{1}^{-1}} \omega_{\pi_{\lambda}} \omega_{\rho_{2}^{-1}}$ é uma trança de permutação positiva e portanto é a trança $\omega_{\pi}$. A chave da demonstração é simplesmente observar que $\omega_{\pi_{\lambda}}$ não cruza linhas associadas à mesma linha do diagrama $\lambda$.

Suponha que haja cruzamento na trança $\omega_{\rho_{1}^{-1}}$ das linhas $k$ e $j$. Como $\rho_{1}^{-1} \in R(\lambda)$, ambas devem ser $\lambda_{i}$-linhas. Por sua vez, $\omega_{\pi_{\lambda}}$, como observamos, não cruza linhas associadas à mesma linha do diagrama $\lambda$. Além disso, como $\pi_{\lambda}$ separa $\lambda$ e $\lambda^{v}$, as linhas consideradas irão acabar em linhas associadas a linhas distintas de $\lambda^{v}$. Por fim, como $\rho_{2}^{-1} \in R\left(\lambda^{v}\right)$, não pode haver cruzamento dessas linhas pela trança de permutação positiva associada.

Usando argumentos completamente análogos, mostramos que sempre que uma das tranças $\omega_{\rho_{1}^{-1}}, \omega_{\pi_{\lambda}}$ ou $\omega_{\rho_{2}^{-1}}$, efetuar o cruzamento de duas linhas distintas, as outras não o fazem.

Logo, como a trança $\omega_{\tau}=\omega_{\rho_{1}^{-1}} \omega_{\pi_{\lambda}} \omega_{\rho_{2}^{-1}}$ só possui cruzamentos positivos, nenhum par de linhas é cruzado mais de uma vez e está associada à permutação $\tau$, pela Proposição 1.4.2 temos que:

$$
\omega_{\tau}=\omega_{\rho_{1}^{-1}} \omega_{\pi_{\lambda}} \omega_{\rho_{2}^{-1}}
$$


Teorema 3.3.3 Seja $\lambda$ um diagrama de Young com $|\lambda|=n$. Então $e_{\lambda}^{2}=\alpha_{\lambda} e_{\lambda}$ para algum escalar $\alpha_{\lambda} \in \Lambda$. Além disso, se $\lambda \neq \mu$, onde $|\mu|$, então $e_{\lambda} e_{\mu}=0$.

\section{Demonstração:}

Seja $e_{\lambda}=E_{\lambda}(a) \omega_{\pi_{\lambda}} E_{\lambda^{v}}(b) \bar{\omega}_{\pi_{\lambda} v}$.Logo:

$$
e_{\lambda}^{2}=E_{\lambda}(a) h E_{\lambda^{v}}(b) \bar{\omega}_{\pi_{\lambda^{v}}}
$$

onde $h=\omega_{\pi_{\lambda}} E_{\lambda^{v}}(b) \bar{\omega}_{\pi_{\lambda^{v}}} E_{\lambda}(a) \omega_{\pi_{\lambda}}$. Pelo Teorema 2.3.1, podemos expressar $h$ como uma combinação linear das tranças de permutação positiva, logo:

$$
h=\sum_{\tau \in S_{n}} \gamma_{\tau} \omega_{\tau}
$$

Sendo assim,

$$
e_{\lambda}^{2}=\sum_{\tau \in S_{n}} \gamma_{\tau} E_{\lambda}(a) \omega_{\tau} E_{\lambda^{v}}(b) \bar{\omega}_{\pi_{\lambda}}
$$

Pelo Lema 3.3.4, se $\tau$ não separa $\lambda$ e $\lambda^{v}$, temos $E_{\lambda}(a) \omega_{\tau} E_{\lambda^{v}}(b)=0$.

Vamos portanto considerar somente $\tau \in S_{n}$ que não separa $\lambda$ e $\lambda^{v}$. Nesse caso, pelo Corolário 3.3.4, $\omega_{\tau}=\omega_{\rho_{1}^{-1}} \omega_{\pi_{\lambda}} \omega_{\rho_{2}^{-1}}$, onde $\rho_{1} \in R(\lambda)$ e $\rho_{2} \in R\left(\lambda^{v}\right)$. Portanto, pelo Lema 3.3.2:

$$
\begin{aligned}
E_{\lambda}(a) \omega_{\rho_{1}^{-1}}= & \phi_{b}\left(\omega_{\rho_{1}^{-1}}\right) E_{\lambda}(a) \\
& e \\
\omega_{\rho_{2}^{-1}} E_{\lambda^{v}}(b)= & \phi_{a}\left(\omega_{\rho_{2}^{-1}}\right) E_{\lambda^{v}}(b) .
\end{aligned}
$$

Seja $\alpha(\tau)=\phi_{b}\left(\omega_{\rho_{1}^{-1}}\right) \phi_{a}\left(\omega_{\rho_{2}^{-1}}\right)$. Logo, considerando $\alpha_{\lambda}=\sum_{\tau \in S_{n}} \alpha(\tau) \gamma_{\tau}$ temos:

$$
e_{\lambda}^{2}=\alpha_{\lambda} e_{\lambda}
$$

como queríamos. Suponha agora $\lambda \neq \mu$. Logo:

$$
e_{\lambda} e_{\mu}=E_{\lambda}(a) \omega_{\pi_{\lambda}} E_{\lambda^{v}}(b) \bar{\omega}_{\pi_{\lambda} v} E_{\mu}(a) \omega_{\pi_{\mu}} E_{\mu^{v}}(b) \bar{\omega}_{\pi_{\mu} v}
$$

Pelo Corolário 3.3.1, como $\lambda \neq \mu$ temos que ou $\lambda$ e $\mu^{v}$ são inseparáveis, ou $\lambda^{v}$ e $\mu$ são inseparáveis. No primeiro caso, pelo Lema 3.3.4:

$$
E_{\lambda^{v}}(b)\left(\bar{\omega}_{\pi_{\lambda^{v}}}\right) E_{\mu}(a)=0 .
$$

No segundo caso, pelo Corolário 3.3.3:

$$
E_{\lambda}(a)\left(\omega_{\pi_{\lambda}} E_{\lambda^{v}}(b) \bar{\omega}_{\pi_{\lambda} v} E_{\mu}(a) \omega_{\pi_{\mu}}\right) E_{\mu^{v}}(b) \bar{\omega}_{\pi_{\mu^{v}}} .
$$

Portanto, em qualquer um dos casos temos $e_{\lambda} e_{\mu}=0$ quando $\lambda \neq \mu$. 


\subsection{Especializando $H_{n}$}

Lembrando que:

$$
\Lambda=\mathbb{C}\left[x, x^{-1}, v, v^{-1}, s, s^{-1}, z, \delta\right] /\left\langle v^{-1}-v=\delta z>\right.
$$

onde $z=s-s^{-1}$, considere $\boldsymbol{g}: \Lambda \rightarrow \mathbb{C}$ um homomorfismo de anéis definido por:

$$
g(x)=g(v)=g(s)=1 .
$$

Observamos que o $g(\delta)$ pode ser a princípio qualquer valor (pois, em qualquer caso a relação é satisfeita). Posteriormente esse valor será definido.

Podemos considerar $\mathbb{C}$ um $\Lambda$-módulo através da ação :

$$
r . w=g(r) . w, \forall r \in \Lambda, \forall w \in \mathbb{C}
$$

Sendo assim, podemos considerar o $\Lambda$-módulo $H_{n} \otimes_{\Lambda} \mathbb{C}$. Por outro lado, $H_{n} \otimes_{\Lambda} \mathbb{C}$ tem uma estrutura de $\mathbb{C}$-álgebra dada pela ação :

$$
w^{\prime}(x \otimes w)=x \otimes w \cdot w^{\prime}, \forall w, w^{\prime} \in \mathbb{C}, \forall x \in H_{n}
$$

com o produto $(h \otimes w)\left(h^{\prime} \otimes w^{\prime}\right)=h . h^{\prime} \otimes w . w^{\prime}$, onde $h, h^{\prime} \in H_{n}$ e $w, w^{\prime} \in \mathbb{C}$.

Lema 3.4.1 $A \mathbb{C}$-álgebra $H_{n} \otimes_{\Lambda} \mathbb{C}$ é gerada pelo conjunto:

$$
\left\{\sigma_{i} \otimes 1: i=1, \ldots, n-1\right\}
$$

onde $\left\{\sigma_{i}: i=1, \ldots, n-1\right\}$ são as tranças elementares geradoras de $H_{n}$.

\section{Demonstração:}

Todo elemento da $\mathbb{C}$-álgebra $H_{n} \otimes_{\Lambda} \mathbb{C}$ pode ser escrito como uma soma finita de elementos do tipo $h \otimes w$, onde $h \in H_{n}$ e $w \in \mathbb{C}$.

Logo basta mostrarmos que todo elemento desse tipo pode ser escrito como um polinômio nos $\sigma_{i}^{\prime} s$ e coeficientes em $\mathbb{C}$.

Temos que $h=\sum_{i=1}^{k} \lambda_{i} p_{i}\left(\sigma_{1}, \ldots, \sigma_{n-1}\right)$ onde $\lambda_{i}$ é um escalar em $\Lambda$ e $p_{i}$ é um monômio nos $\sigma_{i}^{\prime} s$. 
Logo, pelas propriedades do produto tensorial e pela definição da ação de $\Lambda$ em $\mathbb{C}$ :

$h \otimes w=\left(\sum_{i=1}^{k} \lambda_{i} p_{i}\left(\sigma_{1}, \ldots, \sigma_{n-1}\right)\right) \otimes w \Leftrightarrow$

$h \otimes w=\sum_{i=1}^{k} \lambda_{i} p_{i}\left(\sigma_{1}, \ldots, \sigma_{n-1}\right) \otimes w \Leftrightarrow$

$h \otimes w=\sum_{i=1}^{k} p_{i}\left(\sigma_{1}, \ldots, \sigma_{n-1}\right) \otimes g\left(\lambda_{i}\right) w$

Por outro lado, pela ação de $\mathbb{C}$ em $H_{n} \otimes_{\Lambda} \mathbb{C}$ e pela definição do produto nessa álgebra, temos:

$$
\begin{aligned}
& h \otimes w=\sum_{i=1}^{k} g\left(\lambda_{i}\right) w\left(p_{i}\left(\sigma_{1}, \ldots, \sigma_{n-1}\right) \otimes 1\right) \Leftrightarrow \\
& h \otimes w=\sum_{i=1}^{k} g\left(\lambda_{i}\right) w\left(p_{i}\left(\sigma_{1} \otimes 1, \ldots, \sigma_{n-1} \otimes 1\right)\right)
\end{aligned}
$$

Lema 3.4.2 [Cohn] Seja $K$ um anel comutativo e sejam $U$ e $V$ K-módulos com bases $\left\{e_{1}, \ldots, e_{m}\right\}$ e $\left\{f_{1}, \ldots, f_{k}\right\}$, respectivamente, então:

$$
\left\{e_{i} \otimes f_{j} / i=1, \ldots, m \text { e } j=1, \ldots, k\right\}
$$

é uma base para o $K$-módulo $U \otimes V$.

Corolário 3.4.1 $O$ conjunto $\left\{\omega_{\pi} \otimes 1 / \pi \in S_{n}\right\}$ é uma base do $\Lambda$-módulo $H_{n} \otimes_{\Lambda} \mathbb{C}$.

Proposição 3.4.1 $H_{n} \otimes_{\Lambda} \mathbb{C} e \mathbb{C} S_{n}$ são $\mathbb{C}$-álgebras isomorfas.

\section{Demonstração:}

Lembremos primeiramente que a $\mathbb{C}$-álgebra $\mathbb{C} S_{n}$ é gerada por:

$$
\left\{\phi_{1}, \phi_{2}, \ldots, \phi_{n-1}\right\}
$$

com a relações : 
(i) $\phi_{i}^{2}=1, \quad i=1, \ldots, n-1$

(ii) $\phi_{i} \phi_{j}=\phi_{j} \phi_{i}, \quad|i-j|>1$

(iii) $\phi_{i} \phi_{i+1} \phi_{i}=\phi_{i+1} \phi_{i} \phi_{i+1}, \quad i=1, \ldots, n-2$.

onde $\phi_{i}=(i i+1)$.

Além disso, como $\mathbb{C}$-módulo livre, tem base $\left\{\pi / \pi \in S_{n}\right\}$ e portanto dimensão $n$ !. Pelo Lema 3.4.1, $H_{n} \otimes_{\Lambda} \mathbb{C}$ é gerada como $\mathbb{C}$-álgebra por $\left\{\sigma_{i} \otimes 1: i=1, \ldots, n-1\right\}$.

Usando as relações em $H_{n}$ (cap.1), quando $|i-j| \geq 2$ temos:

$$
\left(\sigma_{i} \otimes 1\right)\left(\sigma_{j} \otimes 1\right)=\sigma_{i} \sigma_{j} \otimes 1=\sigma_{j} \sigma_{i} \otimes 1=\left(\sigma_{j} \otimes 1\right)\left(\sigma_{i} \otimes 1\right)
$$

Analogamente, quando $i=1, \ldots, n-2$ :

$\left(\sigma_{i+1} \otimes 1\right)\left(\sigma_{i} \otimes 1\right)\left(\sigma_{i+1} \otimes 1\right)=\left(\sigma_{i+1} \sigma_{i} \sigma_{i+1}\right) \otimes 1$

$=\left(\sigma_{i} \sigma_{i+1} \sigma_{i}\right) \otimes 1$

$=\left(\sigma_{i} \otimes 1\right)\left(\sigma_{i+1} \otimes 1\right)\left(\sigma_{i} \otimes 1\right)$

Para $i=1, \ldots, n-1$ :

$0 \otimes 1=\left(x^{-1} \sigma_{i}-x \sigma_{i}^{-1}-\left(s-s^{-1}\right) .1\right) \otimes 1$

$=\sigma_{i} \otimes g\left(x^{-1}\right)-\sigma_{i}^{-1} \otimes g(x)-1 \otimes g\left(s-s^{-1}\right)$

Como $g$ é homomorfismo de anéis e $g(x)=g(s)=1$ temos:

$$
0 \otimes 1=\sigma_{i} \otimes 1-\sigma_{i}^{-1} \otimes 1
$$

E portanto, $\left(\sigma_{i} \otimes 1\right)^{2}=1$.

Logo mostramos que os geradores de $H_{n} \otimes_{\Lambda} \mathbb{C}$ obedecem às mesmas relações dos geradores de $\mathbb{C} S_{n}$. Vamos mostrar que não há nenhuma relação "a mais" em $H_{n} \otimes_{\Lambda} \mathbb{C}$, provando que o homomorfismo de $\mathbb{C}$-álgebras $f: H_{n} \otimes_{\Lambda} \mathbb{C} \rightarrow \mathbb{C} S_{n}$ definido por:

$$
f\left(\sigma_{i} \otimes 1\right)=(i i+1)
$$

é na verdade um isomorfismo. 
Por construção $f$ é sobrejetor. Vamos agora provar que é uma aplicação injetora, mostrando que como $\mathbb{C}$-módulos livres, ou melhor, espaços vetoriais sobre $\mathbb{C}, H_{n} \otimes_{\Lambda} \mathbb{C}$ e $\mathbb{C} S_{n}$ têm mesma dimensão .

Pelo Corolário anterior $\left\{\omega_{\pi} \otimes 1 / \pi \in S_{n}\right\}$ é base de $H_{n} \otimes_{\Lambda} \mathbb{C}$. Utilizando argumentos semelhantes ao Lema 3.4.1, é fácil verificar que esse conjunto gera $H_{n} \otimes_{\Lambda} \mathbb{C}$ linearmente. Logo a sua dimensão é menor ou igual a $\pi$ !. Por outro lado, como $f$ é sobrejetora, temos que a dimensão de $H_{n} \otimes_{\Lambda} \mathbb{C}$ é maior ou igual a dimensão de $\mathbb{C} S_{n}$ que é $n$ ! e portanto ambos têm a mesma dimensão, o que implica que $f$ é injetora.

Considere a seguinte composta de aplicações $\Gamma$ :

$$
H_{n} \simeq H_{n} \otimes_{\Lambda} \Lambda \stackrel{1 \otimes g}{\rightarrow} H_{n} \otimes_{\Lambda} \mathbb{C} \stackrel{f}{\rightarrow} \mathbb{C} S_{n}
$$

Proposição 3.4.2 Г é um homomorfismo de anéis satisfazendo:

$$
\Gamma\left(r \sigma_{i}\right)=g(r)(i i+1), \forall r \in \Lambda
$$

\section{Demonstração:}

$\Gamma$ é claramente um homomorfismo de anéis. Seja $r \in \Lambda$. Temos que $r \sigma_{i} \in H_{n}$ é mandado pelo isomorfismo $H_{n} \simeq H_{n} \otimes_{\Lambda} \mathbb{C}$ para o elemento $r$. $\left(\sigma_{i} \otimes 1\right)=\sigma_{i} \otimes r$, que por sua vez é mandado pela aplicação $1 \otimes g$ para $\sigma_{i} \otimes g(r)$.

Por outro lado, em $H_{n} \otimes_{\Lambda} \mathbb{C}$, temos $\sigma_{i} \otimes g(r)=g(r) .\left(\sigma_{i} \otimes 1\right)$. Como $f$ é isomorfismo de álgebras, $f\left(g(r) \sigma_{i} \otimes 1\right)=g(r) \phi_{i}$ como queríamos.

Dizemos que $\Gamma$ especializa $H_{n}$ em $\mathbb{C} S_{n}$.

Lema 3.4.3 $\Gamma\left(\omega_{\pi}\right)=\pi, \forall \pi \in S_{n}$.

\section{Demonstração:}


Basta lembrar que as tranças elementares $\sigma_{i}$ geram o grupo de tranças e observar que $\Gamma$ restrito a esse grupo (contido em $H_{n}$ ) é um homomorfismo de grupos.

Proposição 3.4.3 Seja $\lambda$ um diagrama de Young. Então $\Gamma\left(e_{\lambda}\right)=C_{\lambda}$, onde $C_{\lambda}$ é o quase-idempotente associado ao tableau $T(\lambda)$.

\section{Demonstração:}

Seja $D=T(\lambda)$. Lembramos que $C_{\lambda}=A_{D} B_{D}$, onde:

$$
A_{D}=\sum_{p \in P_{D}} p \text { e } B_{D}=\sum_{q \in Q_{D}}(-1)^{l(q)} q
$$

sendo $l(q)$ tamanho da permutação $q$ e $P_{D}$ e $Q_{D}$ os subgrupos de $S_{n}$ que preservam as linhas e as colunas de $D$ respectivamente. Lembramos também que:

$$
e_{\lambda}=E_{\lambda}(a) \omega_{\pi_{\lambda}} E_{\lambda v}(b) \bar{\omega}_{\pi_{\lambda v}}
$$

onde:

$$
E_{\lambda}(a)=a_{\lambda_{1}} \otimes a_{\lambda_{2}} \otimes \cdots \otimes a_{\lambda_{k}}
$$

e também:

$$
\begin{aligned}
& a_{\lambda_{i}}=\sum_{\pi \in S_{\lambda_{i}}}(-a)^{-l(\pi)} \omega_{\pi} \\
& b_{\lambda_{i}}=\sum_{\pi \in S_{\lambda_{i}}}(-b)^{-l(\pi)} \omega_{\pi} .
\end{aligned}
$$

Logo, $E_{\lambda}(a)=\sum_{\pi \in P_{D}}(-a)^{-l(\pi)} \omega_{\pi}$. Como, $a=-x s^{-1}$ e $g$ é homomorfismo de anéis, pelas Proposições 3.4.2 e 3.4.3, temos que:

$$
\Gamma\left(E_{\lambda}(a)\right)=\sum_{\pi \in P_{D}}(-g(a))^{-l(\pi)} \Gamma\left(\omega_{\pi}\right)=\sum_{\pi \in P_{D}} \pi .
$$

Vamos analizar agora $\Gamma\left(\omega_{\pi_{\lambda}} E_{\lambda^{v}}(b) \bar{\omega}_{\pi_{\lambda} v}\right)$. Temos que:

$$
\omega_{\pi_{\lambda}} E_{\lambda^{v}}(b) \bar{\omega}_{\pi_{\lambda^{v}}}=\sum_{\pi \in P_{D^{v}}}(-b)^{-l(\pi)} \omega_{\pi_{\lambda}} \omega_{\pi} \bar{\omega}_{\pi_{\lambda^{v}}} .
$$

Lembrando que $b=x s$ (e portanto $g(b)=1$ ) e que $\bar{\omega}_{\pi^{v}}=\omega_{\pi_{\lambda}}^{-1}$ temos:

$$
\Gamma\left(\omega_{\pi_{\lambda}} E_{\lambda^{v}}(b) \bar{\omega}_{\pi_{\lambda^{v}}}\right)=\sum_{\pi \in P_{D^{v}}}(-1)^{-l(\pi)} \pi_{\lambda} \pi \pi_{\lambda}^{-1}
$$


pelos Lemas 3.4.3 e 3.4.2.

Afirmamos que $Q_{D}=\left\{\pi_{\lambda} p \pi_{\lambda}^{-1} / p \in P_{D^{v}}\right\}$

De fato, considere $p \in P_{D}^{v}$. Temos que $\pi_{\lambda}$ manda as colunas de $D$ nas linhas de $D^{v}: p$ preserva as linhas de $D^{v}$ e $\pi_{\lambda}^{-1}$, por sua vez, leva as linhas de $D^{v}$ nas colunas de $D$. Logo $\pi_{\lambda} p \pi_{\lambda}^{-1} \in Q_{D}$ preserva as colunas de $D$ isto é, $\pi_{\lambda} p \pi_{\lambda}^{-1} \in Q_{D}$.

Considere agora $q \in Q_{D}$. Usando argumentos completamente análogos, temos que $\pi_{\lambda}^{-1} q \pi_{\lambda} \in P_{D^{v}}$.

Seja $\varphi_{\pi_{\lambda}}: S_{n} \rightarrow S_{n}$ definida por $\varphi_{\pi_{\lambda}}(\pi)=\pi_{\lambda} \pi \pi_{\lambda}^{-1}$. Logo $\varphi_{\pi_{\lambda}}$ é um isomorfismo (conjugação por um elemento do grupo) tal que:

$$
\begin{gathered}
\varphi_{\pi_{\lambda}}\left(P_{D^{v}}\right) \subseteq Q_{D} \\
e \\
\varphi_{\pi_{\lambda}}^{-1}\left(Q_{D}\right) \subseteq P_{D^{v}}
\end{gathered}
$$

e portanto $\varphi_{\pi_{\lambda}}\left(P_{D^{v}}\right)=Q_{D}$ o que prova a afirmação . Sendo assim:

$$
\sum_{p \in P_{D^{v}}}(-1)^{-l(p)} \pi_{\lambda} p \pi_{\lambda}^{-1}=\sum_{p \in P_{D^{v}}}(-1)^{-l\left(\pi_{\lambda}^{-1} q \pi_{\lambda}\right)} q .
$$

Como a conjugação não muda o sinal da permutação, temos:

$$
(-1)^{-l\left(\pi_{\lambda}^{-1} q \pi_{\lambda}\right)}=(-1)^{-l(q)}=(-1)^{l(q)}
$$

E portanto:

$$
\Gamma\left(\omega_{\pi_{\lambda}} E_{\lambda^{v}}(b) \bar{\omega}_{\pi_{\lambda} v}\right)=\sum_{q \in Q_{D}}(-1)^{l(q)} q=B_{D}
$$

Consequentemente, $\Gamma\left(e_{\lambda}\right)=C_{\lambda}$. 


\subsection{Substituições no anel $\Lambda$}

Vamos agora fazer substituições no nosso anel:

$$
\Lambda=\mathbb{C}\left[x, x^{-1}, v, v^{-1}, s, s^{-1}, z, \delta\right] /\left\langle v^{-1}-v=\delta z>\right.
$$

onde $z=s-s^{-1}$, as quais serão necessárias posteriormente. Seja $N$ um natural não nulo fixado. Faremos as substituições:

$$
x=s^{-1 / N}, v=s^{-N}, \text { e } \delta=s^{N-1}+s^{N-3}+\cdots+s^{-N+1}
$$

compatíveis com a relação $v-v^{-1}=\delta z$. Observamos que a partir de agora temos um valor definido para $g(\delta)$.

Para garantirmos a inversibilidade do escalar $\alpha_{\lambda}$, e portanto obtermos genuínos idempotentes em $H_{n}$, faremos ainda a substituição $s=e^{h / 2}$. Essas substituições induzem um homomorfismo de anéis, que não alteram os resultados até agora obtidos. Além disso, isso feito, todo elemento de $\Lambda$ será representado por uma série formal de potências em $h$, isto é, $\Lambda=\mathbb{C} \llbracket h \rrbracket$ e o homomorfismo $g: \Lambda \rightarrow \mathbb{C}$ passa a ser definido por $g(h)=0$.

A fim de tratarmos da inversibilidade nesse anel precisamos do seguinte resultado:

Proposição 3.5.1 Em $\Lambda=\mathbb{C} \llbracket h \rrbracket, f \in \Lambda$ é inversível se e somente se o fator constante em $f$ é não nulo, isto é, $g(f) \neq 0$.

\section{Demonstração:}

Seja $f \in \mathbb{C} \llbracket h \rrbracket$. Pela definição do produto de duas séries formais é imediato que se $f$ é inversível, então $g(f) \neq 0$. Para mostrar a outra implicação, considere:

$$
f(X)=\sum_{n=0}^{\infty} a_{n} X^{n}
$$

tal que $g(f)=a_{0} \neq 0$. Vamos construir um elemento:

$$
g(X)=\sum_{n=0}^{\infty} b_{n} X^{n}
$$

tal que $f . g=g \cdot f=1$. 
Seja $f . g(X)=\sum_{n=0}^{\infty} c_{n} X^{n}$, sendo $c_{n}=\sum_{k=0}^{n}(-1)^{k} a_{k} b_{m-k}$. Definimos $b_{0}=a_{0}^{-1} \mathrm{e}$ portanto $c_{0}=1$. Vamos agora "obrigar" os coeficientes $c_{n}$ a se anularem, definindo indutivamente o valor de $b_{n}$ :

$$
0=\sum_{k=0}^{1}(-1)^{k} a_{k} b_{1-k}=a_{0} b_{1}-a_{1} b_{0} \Rightarrow b_{1}=a_{0}^{-1}\left(a_{1} b_{0}\right)
$$

e portanto, se $b_{1}=b_{0}\left(a_{1} b_{0}\right)$ temos $c_{1}=0$.

Assumindo $b_{i}$ definido para $i<m$, definimos $b_{m}$ como:

$$
b_{m}=-b_{0}\left(\sum_{k=1}^{m}(-1)^{k} a_{k} b_{m-k}\right)
$$

o que faz com que $c_{n}$ seja nulo.

Por outro lado, como o anel dos coeficientes (no caso $\mathbb{C}$ ) é comutativo, observamos que se $g \cdot f(X)=\sum_{m=0}^{\infty} d_{m} X^{m}$, temos que:

$$
d_{m}=(-1)^{m} c_{m}, \quad \forall m \in \mathbb{N}
$$

e portanto, $g \cdot f=f \cdot g=1$, como queríamos.

OBS: Essa proposição é claramente verdadeira se trocarmos $\mathbb{C}$ por um anel comutativo com unidade e o termo $\neq 0$ por "inversível".

Lema 3.5.1 O escalar $\alpha_{\lambda}$ tal que $e_{\lambda}^{2}=\alpha_{\lambda} e_{\lambda}$ é inversível em $\Lambda=\mathbb{C} \llbracket h \rrbracket$.

\section{Demonstração:}

Pelo Lema 3.4.2 e pela Proposição 3.4.3 temos:

$$
\Gamma\left(e_{\lambda}^{2}\right)=g\left(\alpha_{\lambda}\right) C_{\lambda}
$$

Por outro lado:

$$
\Gamma\left(e_{\lambda}^{2}\right)=\Gamma\left(e_{\lambda}\right)^{2}=C_{\lambda}^{2}
$$

Como $C_{\lambda}^{2}=a_{\lambda} C_{\lambda}$ temos: 


$$
g\left(\alpha_{\lambda}\right) C_{\lambda}=a_{\lambda} C_{\lambda}
$$

e portanto, $g\left(\alpha_{\lambda}\right)=a_{\lambda}$ escalar não nulo pelo Teorema 3.2.5. Logo, pela Proposição anterior, $\alpha_{\lambda}$ é inversível.

\section{Nota:}

(i) A partir desse momento, estaremos sempre considerando $\Lambda=\mathbb{C} \llbracket h \rrbracket$, porém frequentemente, a fim de facilitarmos alguns cálculos, efetuaremos as substituições após estes.

(ii) O Lema anterior nos permite considerar o elemento $\left(1 / \alpha_{\lambda}\right) e_{\lambda} \in H_{n}$, que é um genuíno idempotente para todo $\lambda$, tal que $|\lambda|=n$. Chamaremos o fecho desse elemento em $C^{+}$de $Q_{\lambda}$.

Usaremos agora a função $\Gamma$ para mostrar que $H_{n}$ uma decomposição útil da álgebra $H_{n}$, onde os elementos $e_{\lambda}$ fazem papel semelhante ao dos elementos $C_{\lambda} \in \mathbb{C} S_{n}$ (lembrando que $C_{\lambda}$ é o quase-idempotente definido a partir do tableau de Young $T(\lambda))$.

Teorema 3.5.1 $H_{n}=\sum_{|\lambda|=n} H_{n} e_{\lambda} H_{n}$.

\section{Demonstração:}

Lembremos que $\mathbb{C} S_{n}$ é gerado como $\mathbb{C}$-módulo por:

$$
\left\{\pi_{i} / i=1, \ldots, n !\right\}
$$

onde $\pi_{i}$ são as transposições elementares e $H_{n}$ é gerado como $\Lambda$-módulo livre por:

$$
\left\{\omega_{i} / i=1, \ldots, n !\right\}
$$

onde podemos supor que $\Gamma\left(\omega_{i}\right)=\pi_{i}$, pelo Lema 3.4.3.

Pela Proposição 3.4.3 e como $\Gamma$ é um homomorfismo de anéis sobrejetor, temos:

$$
\Gamma\left(H_{n} e_{\lambda} H_{n}\right)=\Gamma\left(H_{n}\right) \cdot C_{\lambda} \cdot \Gamma\left(H_{n}\right)=\mathbb{C} S_{n} C_{\lambda} \mathbb{C} S_{n}
$$


E portanto, pela Proposição 3.2.5:

$$
\Gamma\left(\sum_{|\lambda|=n} H_{n} e_{\lambda} H_{n}\right)=\bigoplus_{|\lambda|=n} \mathbb{C} S_{n} C_{\lambda} \mathbb{C} S_{n}=\mathbb{C} S_{n}
$$

Sendo assim, $\exists v_{i} \in \sum_{|\lambda|=n} H_{n} e_{\lambda} H_{n}$ tal que $\Gamma\left(v_{i}\right)=\pi_{i}$.

Como $\left\{\omega_{j} / j=1, \ldots, n\right.$ ! $\}$ geram $H_{n}$ linearmente, temos $v_{i}=\sum_{j=1}^{n !} b_{i j} \omega_{j}$, onde $b_{i j} \in \Lambda$.

Considere a matriz $B=\left(b_{i j}\right)_{n ! \times n !}$. Se mostrarmos que $B$ é inversível, estaremos mostrando que podemos escrever cada $\omega_{j}$ como combinação $\Lambda$-linear dos $v_{j}$ 's e portanto $\omega_{j} \in \sum_{|\lambda|=n} H_{n} e_{\lambda} H_{n}$ para todo $j=1, \ldots, n$ !, isto é, $H_{n}=\sum_{|\lambda|=n} H_{n} e_{\lambda} H_{n}$.

De fato, pela Proposição 3.4.2:

$$
\Gamma\left(v_{i}-\omega_{i}\right)=0 \Longrightarrow\left(g\left(b_{i i}\right)-1\right) \pi_{i}+\sum_{i \neq j} g\left(b_{i j}\right) \pi_{j}=0 .
$$

Como $\left\{\pi_{j} / j=1, \ldots, n\right.$ ! $\}$ é base do $\mathbb{C}$-módulo $\mathbb{C} S_{n}$, temos $g\left(b_{i j}\right)=0$, se $i \neq j$ e $g\left(b_{i i}\right)=1$.

Logo, $b_{i j}$ tem termo constante não nulo (no caso igual a 1 ) se e somente se $i=j$, o que nos permite escrever a matriz $B$ como:

$$
B=I+h B^{\prime}
$$

Como $\operatorname{det} B$ é um polinômio em $\Lambda$ e $g$ é homomorfismo de anéis, temos que $g(\operatorname{det} B)=\operatorname{det}(g(B))=\operatorname{det} I=1$. Segue portanto que o $\operatorname{det} B$ tem termo constante igual a 1 sendo pela Proposição 3.5.1 inversível em $\Lambda$ e consequentemente temos que $B$ é uma matriz inversível. 


\section{Capítulo 4}

\section{A Álgebra dos diagramas de Young}

Vamos agora dar uma estrutura de álgebra ao conjunto dos diagramas de Young e mostrar que obtemos dessa forma, uma outra caracterização de álgebra $C^{+}$.

\subsection{O produto no conjunto dos diagramas}

Definição 4.1.1 Sejam $\mu$ e $\lambda$ diagramas de Young, onde $\mu=\left(\mu_{1}, \ldots, \mu_{m}\right)$ e $\lambda=$ $\left(\lambda_{1}, \ldots, \lambda_{k}\right)$. Uma $\boldsymbol{\mu}$-expansão de $\lambda$ é obtida da seguinte maneira:

(i) Adicione $\mu_{1}$ células à $\lambda$, cada qual associada ao número 1 , de tal forma que cada par dessas células sejam colocadas em colunas distintas do diagrama resultante e de que este seja um diagrama de Young.

(ii) Repita o processo com as linhas $i=1,2, \ldots, m$ sucessivamente, até que tenha adicionado ao diagrama $\mu_{m}$ células associadas ao número $m$.

Seja $\nu$ o diagrama final. Para cada célula de $\nu$, seja $n_{i}$ o número de células associadas a $i$ acima e à direita da célula (incluindo a mesma). A $u$-expansão de $\lambda$ é dita estrita se para qualquer célula de $\nu, i<j$ implica $n_{i} \geq n_{j}$. 
Considere $Y$ o conjunto das combinações formais $\Lambda$-lineares dos diagramas de Young, ou melhor dizendo, o $\Lambda$-módulo com base no conjunto dos diagramas de Young. Podemos tornar $Y$ uma $\Lambda$-álgebra definindo o produto de dois diagramas de Young quaisquer pela fórmula:

$$
\lambda \mu=\sum_{|\nu|=|\lambda|+|\mu|} a_{\lambda \mu}^{\nu} \nu
$$

onde os $a_{\lambda \mu}^{\nu}$ são chamados coeficientes de Littlewood-Richardson e são definidos pelo número de maneiras distintas que o diagrama $\nu$ pode ser obtido através de uma $\mu$-expansão estrita de $\lambda$.

Exemplo: O diagrama $\nu=(3,2,1,1)$, não pode ser obtido de $\lambda=(3,2)$ e $\mu=(2)$ através de uma $\mu$-expansão estrita (pois a duas células da mesma linha de $\mu$ teriam que serem colocadas ambas na mesma coluna de $\lambda$ ). Logo, o coeficiente de $\nu$ no produto $\lambda \mu$ é $a_{\lambda \mu}^{\nu}=0$.

Obviamente o produto está bem definido e tem por identidade a partição nula, porém é através da Teoria de Representações da álgebra de Lie $s l(N)$, que esse produto se revela associativo e comutativo. Usaremos essas propriedades sem demonstração, mas detalhes sobre esses coeficientes na Teoria de Representações de $\operatorname{sl}(N)$ podem ser vistos em [FH].

Nota: O produto assim definido vem da relação de $Y$ com a anel das classes de representação de $\operatorname{sl}(N)$ (cujo produto, por sua vez, é definido a partir do produto tensorial), relação essa que podemos ver no seguinte resultado:

Proposição 4.1.1 [FH] Sejam $V_{\lambda}$ e $V_{\mu}$ duas representações irredutíveis de $U(\operatorname{sl}(N))$. Então $V_{\lambda} \otimes V_{\mu}=\sum_{|\nu|=|\lambda|+|\mu|} a_{\lambda \mu}^{\nu} V_{\nu}$, onde $a_{\lambda \mu}^{\nu}$ são os coeficientes de Littlewood-Richardson.

\section{2 $Y$ e $C^{+}$são álgebras isomorfas}

Vamos agora detalhar mais um pouco a estrutura da álgebra $Y$. Observamos primeiramente que o produto definido, $Y$ torna-se um anel graduado, isto é:

$$
Y=\bigoplus_{n=0}^{\infty} Y^{(n)}
$$


onde $Y^{(n)}$ é o espaço $\Lambda$-linear gerado pelos diagramas com exatamente $n$-células.

Proposição 4.2.1 Y é gerado como anel polinomial pelos diagramas de Young com uma única coluna.

\section{Demonstração:}

Indução no número de colunas e no número de células na última coluna. Para $\lambda$ com uma única coluna e $k$ células o resultado é imediato.

Assuma, portanto, que o resultado é válido para todos os diagramas de Young com no máximo $m$ colunas e menos que $k$ células na última coluna. Seja $\lambda$ tal que $\lambda^{v}=\left(\lambda_{1}^{v}, \ldots, \lambda_{m}^{v}\right)$ com $\lambda_{m}^{v}=k$. Seja $\mu$ o diagrama obtido removendo a última coluna de $\lambda$.

Como $\mu$ tem $m-1$ colunas, pela hipótese de indução, existe uma expressão polinomial de $\mu$ em termos dos diagramas de uma única coluna. Temos que $\lambda$ é uma das parcelas do produto $\mu c_{k}$, isto é:

$$
\mu c_{k}=\sum_{|\nu|=|\mu|+\left|c_{k}\right|, \nu \neq \lambda} a_{\mu c_{k}}^{\nu} \nu+a_{\mu c_{k}}^{\lambda} \lambda
$$

A única maneira de obtermos $\lambda$ por uma $c_{k}$-expansão de $\mu$ é adicionando a única célula de cada linha $i$ de $c_{k}$ à linha $i$ de $\mu$. Logo, $a_{\mu c_{k}}^{\lambda}=1$ e portanto:

$$
\lambda=\mu c_{k}-\sum_{|\nu|=|\mu|+\left|c_{k}\right|, \nu \neq \lambda} a_{\mu c_{k}}^{\nu} \nu .
$$

Segundo as regras da expansão estrita, as outras parcelas possíveis devem ter no máximo $m$ colunas e no máximo $k-1$ células na $m$-ésima coluna.

Sendo assim, pela hipótese de indução, cada $\nu$ que aparece na soma com $a_{\mu c_{k}}^{\nu} \neq 0$ é escrito como uma expressão polinomial de diagramas de uma só coluna, o que implica que o resultado é válido para $\lambda$.

\subsubsection{Nota: Fórmula de Giambelli}

Seja $c_{i}$ o diagrama com uma única coluna de $i$ células e $\lambda=\left(\lambda_{1}, \ldots, \lambda_{k}\right)$ um diagrama de Young tal que $\lambda_{1}=m$ (isto é, o seu conjugado $\lambda^{v}$ possui $m$ linhas). Há uma 
fórmula, a chamada fórmula de Giambelli, que descreve $\lambda$ como um polinômio nos diagramas $c_{i}{ }^{\prime} s$ a qual é dada por:

$$
\lambda=\left|\begin{array}{cccc}
c_{\lambda_{1}^{v}} & c_{\lambda_{1}^{v}+1} & \cdots & c_{\lambda_{1}^{v}+m-1} \\
c_{\lambda_{2}^{v}-1} & c_{\lambda_{2}^{v}} & \cdots & c_{\lambda_{2}^{v}+m-2} \\
\vdots & \vdots & \ddots & \vdots \\
c_{\lambda_{m}^{v}-m+1} & c_{\lambda_{m}^{v}-m+2} & \cdots & c_{\lambda_{m}^{v}}
\end{array}\right|
$$

Observamos ainda que todo diagrama que aparece na expressão de $\lambda$ por essa fórmula tem exatamente o mesmo número de células de $\lambda$ (fato esse que decorre da própria expressão dada acima e da definição de produto em $Y$ ). Para maiores detalhes, consultar $[\mathrm{FH}]$.

Considere $R_{\infty}=\Lambda\left[C_{1}, C_{2}, \ldots, C_{i}, \ldots\right]$, o anel dos polinômios em uma quantidade infinita e enumerável de indeterminadas.

Proposição $4.2 .2 R_{\infty}$ e $C^{+}$são $\Lambda$-álgebras isomorfas.

\section{Demonstração:}

Basta observar que ambas são álgebras livres em um número infinito e enumerável de geradores.

Definição 4.2.1 Definimos o peso de um monômio em $r \in R_{\infty}, r=C_{i_{1}}^{j_{1}} C_{i_{2}}^{j_{2}} \ldots C_{i_{m}}^{j_{m}}$ como $\sum_{k=1}^{m} i_{k} j_{k}$

Lema 4.2.1 Seja $R_{\infty}^{(n)}$ o $\Lambda$-submódulo de $R_{\infty}$ gerado pelos monômios de peso $n$. Então $R_{\infty}$ é a soma direta desses espaços, isto é, $R_{\infty}=\bigoplus_{n=0}^{\infty} R_{\infty}^{(n)}$. Além disso, se $r^{(i)} \in R_{\infty}^{(i)}$ e $r^{(j)} \in R_{\infty}^{(j)}$, então $r^{(i)} r^{(j)} \in R_{\infty}^{(i+j)}$.

\section{Demonstração:}


Todo monômio de $R_{\infty}$ está em $R_{\infty}^{(n)}$ para algum $n \in \mathbb{N}$ e a soma desses espaços é direta pela própria definição destes. Logo claramente temos:

$$
R_{\infty}=\bigoplus_{n=0}^{\infty} R_{\infty}^{(n)}
$$

A outra afirmação decorre de forma imediata da definição de peso de um monômio.

Finalmente, mostraremos que a álgebra dos diagramas de Young é isomorfa à álgebra $C^{+}$dos diagramas no anel.

Lema 4.2.2 Dado $n \in \mathbb{N}$, o número de monômios de peso $n$ em $R_{\infty}$, isto é, a dimensão de $R_{\infty}^{(n)}$, é igual ao número de partições de $n$.

\section{Demonstração:}

Basta mostrar que há uma bijeção entre o conjunto dos monômios de peso $n$ em $R_{\infty}$ e o conjunto dos diagramas de Young com $n$ células. De fato, seja $C_{i_{1}}^{j_{1}} C_{i_{2}}^{j_{2}} \ldots C_{i_{m}}^{j_{m}}$ tal que $\sum_{k=1}^{m} i_{k} j_{k}=n$. Podemos assumir, bastando efetuar uma reordenação dos $C_{i}{ }^{\prime} s$, que $i_{1} \geq i_{2} \geq \ldots \geq i_{m}$. Sendo assim, a cada monômio em $R_{\infty}^{(n)}$ podemos associar um diagrama de Young $\lambda$ com $j_{k}$ linhas de $i_{k}$ células e portanto $|\lambda|=n$.

Reciprocamente, dado um diagrama de Young $\lambda$ com $n$ células, definimos unicamente, usando a mesma regra, um monômio de peso $n$.

Proposição 4.2.3 $R_{\infty}$ e Y são $\Lambda$-álgebras isomorfas.

\section{Demonstração:}

Seja $f: R_{\infty} \rightarrow Y$ o homomorfismo de álgebras definido por:

$$
f\left(C_{i}\right)=c_{i}
$$

A aplicação é sobrejetora pela Proposição 4.2.1. Além disso, temos:

$$
f\left(R_{\infty}^{(n)}\right)=Y^{(n)}
$$

decorrendo diretamente da definição do produto em $Y$. Essa igualdade implica que a imagem do conjunto dos monômios de peso $n$ ( base de $R_{\infty}^{(n)}$ ) gera $Y^{(n)}$. 
Por sua rez, $Y^{(n)}$ é gerado como $\Lambda$-módulo pelos diagramas de $n$ células e portanto sua dimensão é o número de partições de $n$, ou equivalentemente, o número de diagramas de Young com $n$ células. Pelo Lema anterior, temos uma aplição linear sobrejetora entre os $\Lambda$-módulos $Y^{(n)}$ e $R_{\infty}^{(n)}$ de mesma dimensão. Como $\Lambda$ é anel comutativo, $f$ é injetora em $R_{\infty}^{(n)}$. Pelo Lema 4.2.1, $f$ é injetora em todo o espaço $R_{\infty}$.

Corolário 4.2.1 $C^{+}$e $Y$ são $\Lambda$-álgebras isomorfas.

\section{Demonstração:}

Decorre da Proposição 4.2.2.

Vamos posteriormente explicitar esse isomorfismo e verificar algumas propriedades as quais nos serão necessárias.

Definições 4.2.1 Seja $N$ um natural não nulo. Considere $R_{N}$ o anel quociente:

$$
R_{N}=R_{\infty} / I
$$

onde $I$ é o ideal gerado por $\left\{c_{k}=0, \forall k>N\right\}$. É imediato que $R_{N} \simeq \Lambda\left[C_{1}, \ldots, C_{N}\right]$. Denotaremos o homormorfismo quociente por $p_{N}: R_{\infty} \rightarrow R_{N}$.

Vamos agora mostrar uma relação entre séries formais com coeficientes em $Y$, que serão úteis na obtenção de algumas igualdades. Antes porém, vamos examinar com detalhes o produto $c_{k} d_{l}$, onde $c_{k}$ é o diagrama coluna de $k$ células e $d_{l}$ é o diagrama linha de $l$ células.

Quais são os diagramas que podem ser obtidos desse dois diagramas, através de uma expansão estrita? Seja $\mu_{k, l}$ o diagrama:

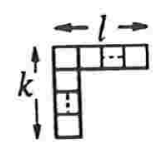

É fácil ver que existem apenas duas possibilidades de uma $d_{l}$-expansão estrita de 
$c_{k}$, quando $l$ e $k$ são não nulos, a saber os diagramas $\mu_{k, l+1}$ e $\mu_{k+1, l}$, ambos obtidos de uma única maneira.

Por outro lado, quando $k$ ou $l$ é nulo, obtemos o diagrama vazio que é o elemento identidade em $Y$. Sendo assim, o produto de $c_{k} d_{l}$ (ver seção 4.1) é dado por:

(i) $c_{k}$, se $\mathrm{l}=0$.

(ii) $d_{l}$, se $\mathrm{k}=0$.

(iii) $\mu_{k+1, l}+\mu_{k, l+1}$, se $l, k>0$.

Sejam $A(X)=\sum_{k=0}^{\infty} a_{k} X^{k}$ e $B(X)=\sum_{l=0}^{\infty} b_{l} X^{l}$ séries formais com coeficientes em um anel comutativo $K$. Lembramos que o produto entre esses dois elementos é definido por:

$$
A(X) B(X)=\sum_{m=0}^{\infty} h_{m} X^{m}
$$

onde $h_{m}=\sum_{k=0}^{m}(-1)^{k} a_{k} b_{m-k}$.

Considere $C(X)=\sum_{k=0}^{\infty}(-1)^{k} c_{k} X^{k}$ e $D(X)=\sum_{l=0}^{\infty}(-1)^{l} d_{l} X^{l}$ duas séries formais com coeficientes em $Y$. Então vale:

Proposição $4.2 .4 C(X) D(X)=1$.

\section{Demonstração:}

Seja $C(X) D(X)=\sum_{m=0}^{\infty} a_{m} X^{m}$. Pela definição do produto entre $C(X)$ e $D(X)$, temos que :

$$
a_{0}=c_{0} d_{0}=1
$$

Por outro lado, quando $m>0$, temos: 


$$
\begin{aligned}
& a_{m}=\sum_{k=0}^{m}(-1)^{k} c_{k} d_{m-k} \\
= & d_{m}+\sum_{k=1}^{m-1}\left((-1)^{k}\left(\mu_{k+1, m-k}+\mu_{k, m-k+1}\right)\right)+(-1)^{m} c_{m} \\
= & d_{m}+\sum_{k=2}^{m}(-1)^{k-1} \mu_{k, m-k}+\sum_{k=1}^{m-1}(-1)^{k-1} \mu_{k, m-k+1}+(-1)^{m} c_{m} \\
= & d_{m}+(-1)^{m-1} \mu_{m, 1}+(-1) \mu_{1, m}+(-1)^{m} c_{m}+\sum_{k=2}^{m-1}\left((-1)^{k-1}\left(\mu_{k, m-k+1}-\mu_{k, m-k+1}\right)\right) \\
= & d_{m}-d_{m}+(-1)^{m-1}\left(c_{m}-c_{m}\right)-0=0
\end{aligned}
$$

Nota: Vamos olhar essa relação no anel quociente $R_{N}$, considerando já o resultado $R_{\infty} \simeq Y$ e portanto, fazendo a identificação $C_{i}=c_{i}$.

Seja $C_{N}(X)=p_{N}(C(X))$. Logo $C_{N}(X)=1-c_{i} X+\cdots+(-1)^{N} c_{N} X^{N}$. Esse polinômio pode ser formalmente fatorado da seguinte forma:

$$
C_{N}(X)=\prod_{i=1}^{N}\left(1-x_{i} X\right)
$$

considerando $c_{k}$ a $k$-ésima função simétrica elementar nas variáveis $\left\{x_{i}\right\}_{i=1}^{N}$. Logo, o anel $R_{N}$ pode ser pensado como o anel dos polinômios simétricos em $\left\{x_{i}\right\}_{i=1}^{N}$.

Definição 4.2.2 Seja $Y_{N}$ o anel quociente $Y / J$, onde $J$ é o ideal gerado por:

$$
\left\{\lambda=\left(\lambda_{1}, \ldots, \lambda_{k}\right) / k>N\right\} .
$$

Proposição $4.2 .5 R_{N}$ é isomorfo $a Y_{N}$.

\section{Demonstração:}

Lembramos que $R_{N}=R_{\infty} / I$, onde $I$ é o ideal gerado por $\left\{c_{k}=0, k>0\right\}$ e $R_{\infty} \simeq Y$ (Proposição 4.2.3). Fazendo uso dessa identificação, vamos mostrar que os ideais $I$ e $J$ são os mesmos e portanto os anéis quocientes também o são .

Observamos primeiramente que $c_{i}$, quando $i>N$, possui mais que $N$ linhas e portanto $I \subseteq J$. Considere agora $\lambda \in J$. Pela Observação 4.2.1, $\lambda$ é expresso pela 
fórmula de Giambelli:

$$
\lambda=\left|\begin{array}{cccc}
c_{\lambda_{1}^{v}} & c_{\lambda_{1}^{v}+1} & \cdots & c_{\lambda_{1}^{v}+m-1} \\
c_{\lambda_{2}^{v}-1} & c_{\lambda_{2}^{v}} & \cdots & c_{\lambda_{2}^{v}+m-2} \\
\vdots & \vdots & \ddots & \vdots \\
c_{\lambda_{m}^{v}-m+1} & c_{\lambda_{m}^{v}-m+2} & \cdots & c_{\lambda_{m}^{v}}
\end{array}\right|
$$

Observe que os elementos da primeira linha do determinante pertencem à $I$, quando $\lambda$ tem mais de $N$ linhas e portanto $\lambda_{1}^{v}>N$. Expandindo o determinante por essa linha, temos que $\lambda \in I$.

Queremos agora explicitar o isomorfismo entre $C^{+}$e $Y$, mostrando que os elementos $\lambda \in Y$ correspondem aos elementos $Q_{\lambda}=\left(1 / \alpha_{\lambda}\right) \widehat{e_{\lambda}} \in C^{+}$. Mas antes, vamos efetuar alguns cálculos envolvendo os elementos $e_{\lambda} \in H_{|\lambda|}$ e $Q_{\lambda} \in C^{+}$.

\subsection{Algumas relações entre os quase-idempotentes de $H_{n}$}

A fim de simplicarmos a notação, denotaremos o quase-idempotente associado ao diagrama $\mu_{k, l}$ por $e_{k, l}$, bem como o escalar associado por $\alpha_{k, l}$ e o fecho em $C^{+}$por $Q_{k, l}$.

Definição 4.3.1 Seja $n \in \mathbb{N}$. Definimos $[n]$ o número quântico $n$ por:

$$
[n]=\frac{s^{n}-s^{-n}}{s-s^{-1}}
$$

Definimos também o fatorial do número quântico $n$ por:

$$
[n] !=[n] .[n-1] \cdot[n-2] \ldots[1]
$$

Observamos que $[n]$ a princípio não pertence ao anel $\Lambda$, por inexistirem nesse último potências negativas de $\left(s^{i}-s^{-i}\right)$ para $i \geq 1$. Manipularemos esses elementos como se estivéssemos em um anel estendido e posteriormente mostraremos que os números quânticos pertencem à $\Lambda$ e são inversíveis nesse anel. 
Proposição 4.3.1 Valem as seguintes relações:

(i) $e_{k^{\prime}, 1} \cdot e_{k, 1}=\phi_{a}\left(e_{k, 1}\right) e_{k^{\prime}, 1}$, se $k^{\prime} \geq k$.

(ii) $e_{1, l^{\prime}} \cdot e_{1, l}=\phi_{b}\left(e_{1, l}\right) e_{1, l^{\prime}}$, se $l^{\prime} \geq l$.

(iii) $\phi_{a}\left(e_{k, 1}\right)=\alpha_{k, 1} \quad$ e $\phi_{b}\left(e_{1, l}\right)=\alpha_{1, l}$.

onde $\phi_{a}$ e $\phi_{b}$ são os homomorfismos lineares definidos no Teorema 3.3.2.

\section{Demonstração:}

Basta ver que $e_{k, 1}=b_{k}$ e $e_{1, l}=a_{l}$ e aplicar diretamente o Teorema 3.3.2. De fato,

$$
e_{1, l}=E_{\lambda}(a) \omega_{\pi_{\lambda}} E_{\lambda^{v}}(b) \bar{\omega}_{\pi_{\lambda^{v}}},
$$

onde $\lambda=\mu_{1, l}$. Nesse caso temos $\pi_{\lambda}$ a permutação identidade e como $\pi_{\lambda^{v}}=\pi_{\lambda}^{-1}$, temos que $\pi_{\lambda}^{v}$ também o é. Logo, $\omega_{\pi_{\lambda}}$ e $\omega_{\pi_{\lambda} v}$ são tranças triviais em $l$ linhas. Sendo assim, temos que $e_{1, l}=E_{\mu_{1, l}}(a)=a_{l}$. Analogamente, concluimos que $e_{k, 1}=b_{k}$.

Convenção: Para simplificar a notação, vamos representar diagramaticamente o elemento $a_{k}$ como um bloco branco com um $k$ no centro. Para diferenciar, vamos representar $b_{k}$ de maneira análoga, só que com um bloco escurecido, ao invés do branco.

Lema 4.3.1 Seja $\omega_{\pi}$ uma $k$-trança de permutação positiva associada à permutação $\pi \in S_{n}$ tal que $\pi(k)=i$, para algum $1 \leq i \leq k$ fixo. Então, $\omega_{\pi}$ pode ser escrita como um produto de tranças de permutação positiva $\omega_{\pi}=\omega_{\pi^{\prime}} \cdot \omega_{\pi_{i}}$, onde $\omega_{\pi^{\prime}}$ é tal que $\pi^{\prime}(k)=k$ e $\omega_{\pi_{i}}$ é uma trança com $k-i$ cruzamentos positivos.

\section{Demonstração:}

Seja $\omega_{\pi}$ uma trança de permutação positiva tal que $\pi(k)=i$. Definimos $\pi^{\prime} \in S_{k}$ como:

$$
\pi^{\prime}(j)= \begin{cases}\pi(j) & , \quad 1 \leq \pi(j)<i \\ k & , \quad j=k \\ \pi(j)-1 & , \quad i<\pi(j)<k\end{cases}
$$


Considere $\pi_{i}=(i i+1 \cdots k) \in S_{k}$. Pela definição das tranças de permutação positiva (ver cap.1) temos:

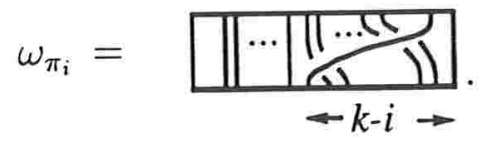

Provaremos o Lema mostrando que $\omega_{\pi^{\prime}} \omega_{\pi_{i}}$ é uma trança de permutação positiva associada à permutação $\pi$ e portanto, pela Proposição 1.4 .2 , é a trança $\omega_{\pi}$. Observe que $\omega_{\pi^{\prime}} \omega_{\pi_{i}}$ é uma trança somente com cruzamentos positivos e tal que nenhum par de linhas se cruzam mais de uma vez, pois em $\omega_{\pi^{\prime}}$ a $k$-ésima linha não se cruza com nenhuma outra e em $\omega_{\pi_{i}}$ os únicos cruzamentos ocorrem exatamente com a $k$-ésima linha. Logo, $\omega_{\rho}=\omega_{\pi^{\prime}} \omega_{\pi_{i}}$ é uma trança de permutação positiva, com $\rho=\pi^{\prime} \cdot \pi_{i}$ (lembrando que pela nossa convenção aplicamos $\pi^{\prime}$ e depois $\pi_{i}$ ). Sendo assim, temos:

$$
\rho(j)= \begin{cases}\pi^{\prime}(j) & , \quad 1 \leq \pi^{\prime}(j)<i \\ \pi^{\prime}(j)+1 & , i \leq \pi^{\prime}(j)<k \\ i & , \quad \pi^{\prime}(j)=k\end{cases}
$$

E portanto:

$$
\rho(j)= \begin{cases}\pi(j) & , \quad 1 \leq \pi^{\prime}(j)<i \\ \pi(j)-1+1 & , i \leq \pi^{\prime}(j)<k \\ 1=\pi(k) & , \quad j=k\end{cases}
$$

Logo $\rho=\pi$.

Lembrando da operação $\otimes$, induzida pela operação geométrica de identificação dos lados de $S\left(\mathbb{R}_{n}^{n}\right)$ (definida da seção 2.3), vamos provar um Lema que será extremamente útil na manipulação dos elementos $e_{\lambda} \in H_{n}$.

Lema 4.3.2 Valem as seguintes relações:

$$
\text { (i) } e_{1, l}=e_{1, l-1} \otimes e_{1,1}+\sum_{i=0}^{l-2}\left(x^{-1} s\right)^{i+1}
$$


(ii) $e_{k, 1}=e_{k-1,1} \otimes e_{1,1}+\sum_{i=0}^{l-2}\left(-x^{-1} s^{-1}\right)^{i+1}$

\section{Demonstração:}

Lembrando raciocínio já feito na Proposição 4.3.1, temos que $e_{1, l}=a_{l}$ e $e_{k, 1}=b_{k}$. Vamos fazer os cálculos para o caso $e_{k, 1}$, sendo estes totalmente análogos para o outro caso (mudando apenas as constantes envolvidas). Aplicando o Lema anterior para $e_{k, 1}$ e lembrando que $b=x s$, temos:

$$
\begin{aligned}
& e_{k, 1}=b_{k}=\sum_{\pi \in S_{k}}\left(-b^{-1}\right)^{l(\pi)} \omega_{\pi} \\
& =\sum_{\pi \in S_{k}, \pi(k)=1}\left(-b^{-1}\right)^{l(\pi)} \omega_{\pi}+\ldots+\sum_{\pi \in S_{k}, \pi(k)=k}\left(-b^{-1}\right)^{l(\pi)} \omega_{\pi} \\
& =\sum_{\pi^{\prime} \in S_{k-1}}\left(-b^{-1}\right)^{l(\pi)}\left(\omega_{\pi^{\prime}} \otimes 1\right) \cdot \omega_{\pi_{1}}+\ldots+\sum_{\pi^{\prime} \in S_{k-1}}\left(-b^{-1}\right)^{l(\pi)}\left(\omega_{\pi^{\prime}} \otimes 1\right) \cdot \omega_{\pi_{k}}
\end{aligned}
$$

onde $\pi=\pi^{\prime} . \pi_{i}$.

Como cada $\omega_{\pi_{i}}$ tem $k-i$ cruzamentos positivos, lembrando que:

$$
l(\pi)=\text { número de auto-enlaçamento de } \omega_{\pi}
$$

temos $l\left(\pi^{\prime}\right)=l(\pi)-(k-i)$ e portanto, da última equação tiramos:

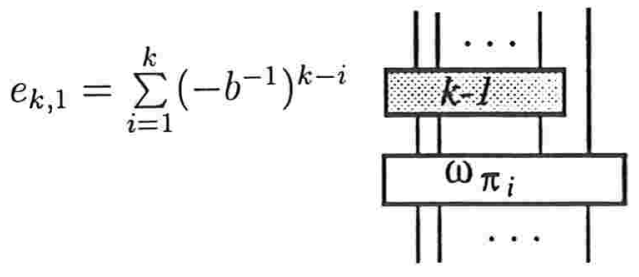

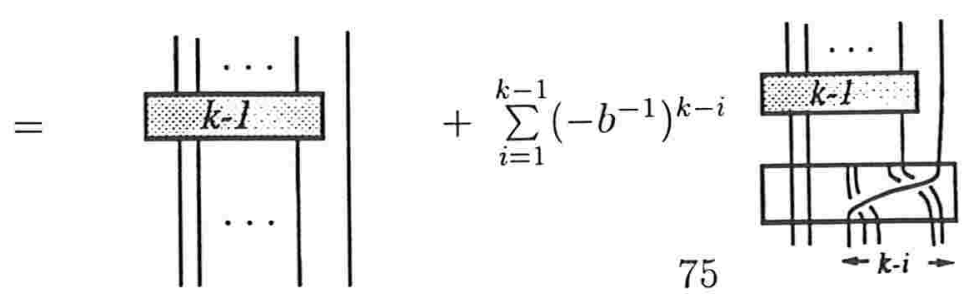


$=\frac{|| \cdots \mid}{\| \frac{k-1}{\mid} \mid}$

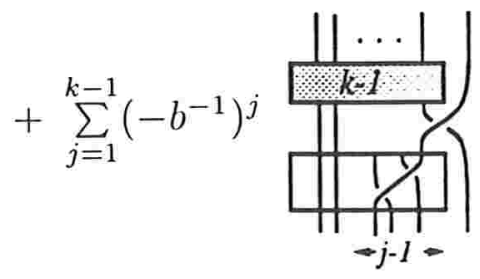

$=e_{k-1,1} \otimes e_{1,1}+\sum_{i=0}^{k-2}\left(-b^{-1}\right)^{i+1}$

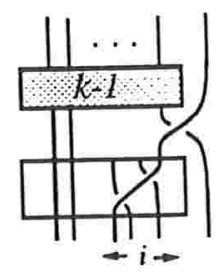

Proposição 4.3.2 Os escalares associados aos quase-idempotentes $e_{k, 1}$ e $e_{1, l}$ são dados por:

$$
\begin{aligned}
\alpha_{k, 1} & =\frac{[k] !}{s^{k(k-1) / 2}} \\
\alpha_{1, l} & =\frac{[l] !}{s^{-l(l-1) / 2}} .
\end{aligned}
$$

\section{Demonstração:}

Vamos demonstrar o resultado por indução em $k$ para o caso $e_{k, 1}$. Novamente, o outro caso sai de forma completamente análoga. Para $k=1, \mu_{1,1}$ é a trança trivial e $\alpha_{1,1}=\frac{1}{s^{0}}=1$ como esperávamos. Considere agora a proposição válida para $1 \leq k^{\prime}<k$. Pelo Lema 4.3.2, temos:

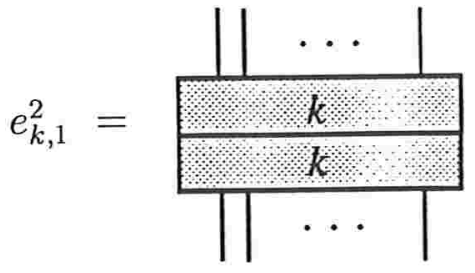




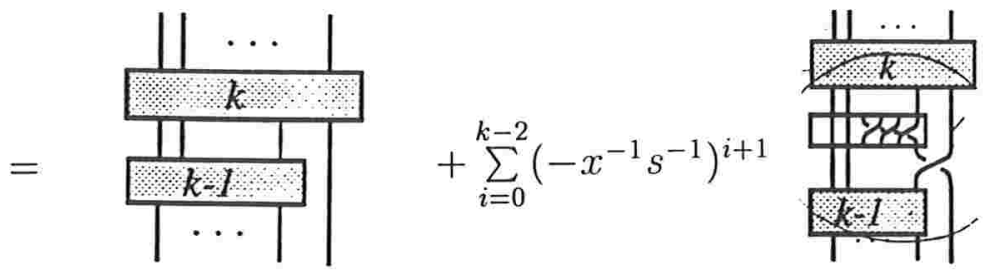

Como $b_{k}$ é central em $H_{k}$ e $a=\left(-x s^{-1}\right.$ ), pelo Teorema 3.3.2, $\phi_{a}\left(\omega_{i}\right)=a^{i+1}$ (pois é produto de $i+1$ tranças elementares) temos:

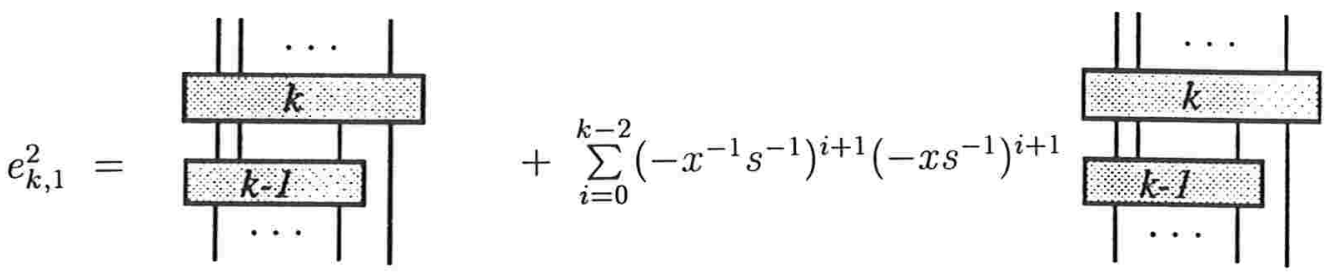

Como $\phi_{a}\left(b_{k-1}\right)=\phi_{a}\left(e_{k-1,1}\right)=\alpha_{k-1,1}$, pelo Teorema 3.3 .2 temos :

$$
e_{k, 1}^{2}=\alpha_{k-1,1} \cdot e_{k, 1}+\sum_{i=0}^{k-2}\left(-x^{-1} s^{-1}\right)^{i+1}\left(-x s^{-1}\right)^{i+1} \alpha_{k-1,1} e_{k, 1}
$$

Pela hipótese de indução :

$$
\begin{aligned}
& e_{k, 1}=\left(\frac{[k-1] !}{s^{\left(k^{2}-3 k+2\right) / 2}}+\frac{[k-1] !}{s^{\left(k^{2}-3 k+2\right)}} \sum_{i=0}^{k-2} s^{-2 i-2}\right) e_{k, 1} \\
& \text { Observando que } \sum_{i=0}^{k-2} s^{-2 i-2}=s^{-k}\left(\frac{s^{k-1}-s^{-k+1}}{s-s^{-1}}\right)=s^{-k}[k-1] \text { temos: } \\
& e_{k, 1}^{2}=\frac{[k-1] !}{s^{\left(k^{2}-k\right) / 2}}\left(\frac{1+s^{-k}[k-1]}{s^{-k+1}}\right) e_{k, 1} \\
& =\frac{[k-1] !}{s^{\left(k^{2}-k\right) / 2}}\left(\frac{\left(s-s^{-1}\right)+s^{-k}\left(s^{k-1}-s^{-k+1}\right)}{\left(s-s^{-1}\right) s^{-k+1}}\right) e_{k, 1} \\
& =\frac{[k] !}{s^{k(k-1) / 2}} e_{k, 1}
\end{aligned}
$$

Corolário 4.3.1 Os números quânticos pertencem à $\Lambda$ e são inversíveis nesse anel.

\section{Demonstração:}


Faremos indução em $k$, usando o fato de que $\alpha_{k, 1}$ é inversível em $\Lambda$. Para $k=2$, temos pela Proposição anterior:

$$
[2]=\alpha_{2,1} \cdot s
$$

e portanto, $[2] \in \Lambda$ e possui inverso [2] $]^{-1}=s^{-1} \alpha_{2,1}^{-1}$. Suponha que para todo $k<n$, $[k] \in \Lambda$ e seja inversível. Nesse caso, $[k] ! \in \Lambda$ e também é inversível. Sendo assim, novamente pela Proposição anterior:

$$
[k+1]=\frac{\alpha_{k+1,1} s^{(k+1) k / 2}}{[k] !}
$$

e portanto, $[k+1] \in \Lambda$ e é inversível nesse anel.

Proposição 4.3.3 O escalar associado ao quase-idempotente $e_{k, l}$ é dado por:

$$
\alpha_{k, l}=\frac{[k+l-1][k-1] ![l-1] !}{s^{(k(k-1)-l(l-1)) / 2}}
$$

\section{Demonstração:}

Observamos primeiramente que:
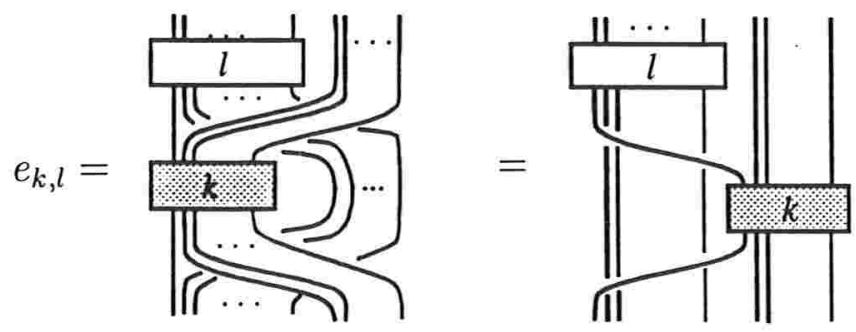

Aplicando o Lema 4.3.2:
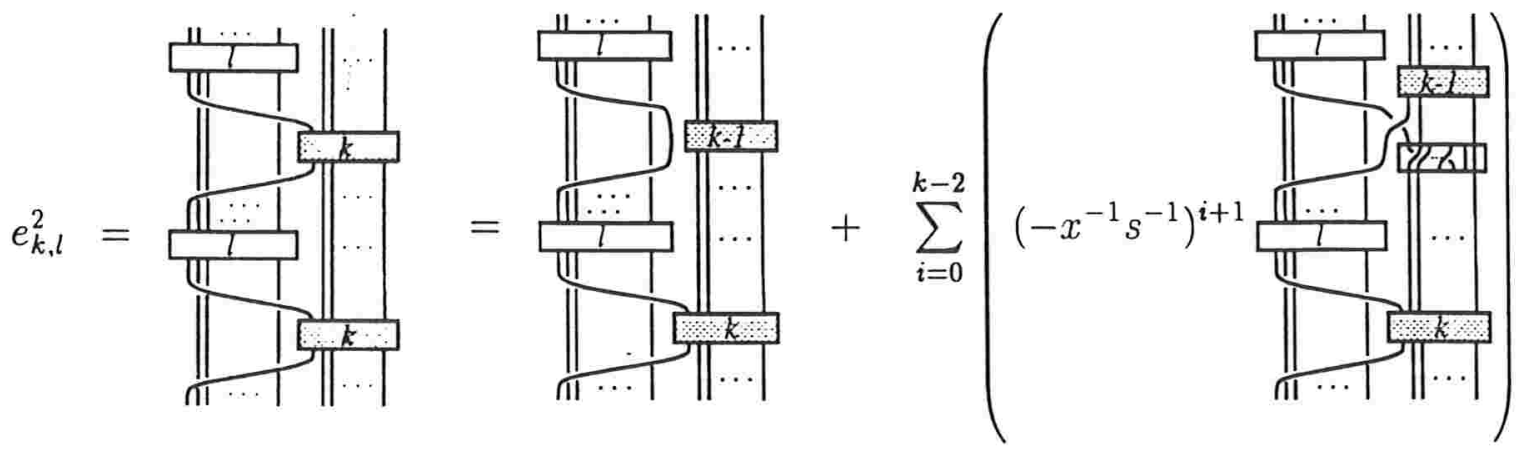
Aplicando agora o Teorema 3.3.2, temos:

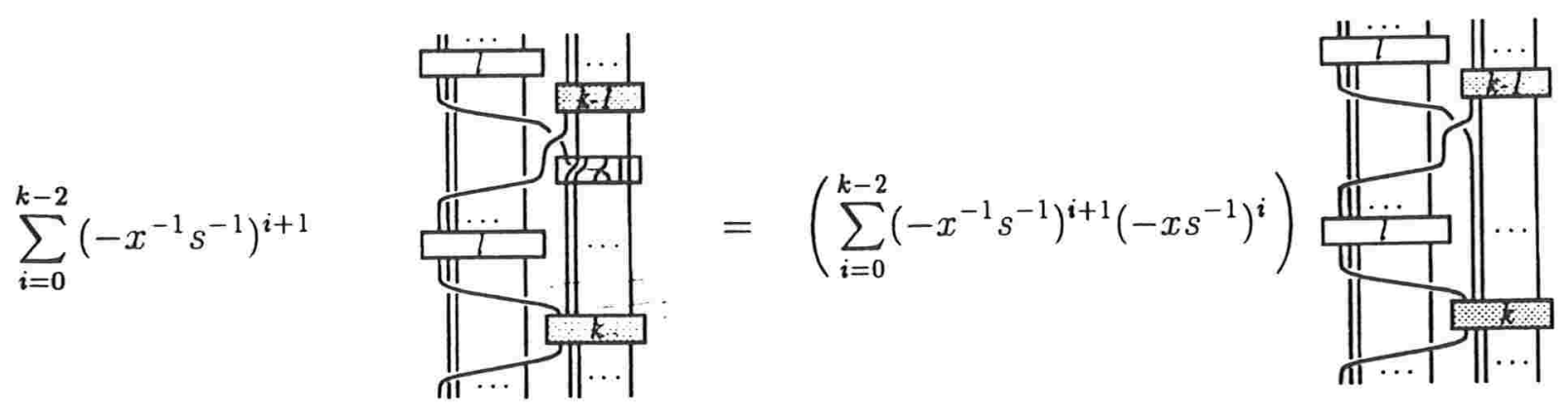

$$
=-x^{-1}\left(\sum_{i=0}^{k-2} s^{-2 i-1}\right)(x s)^{l-1}(x s)^{-(l-1)}
$$

sendo que o coeficiente do último diagrama pode ser simplificado:

$$
x^{-1}\left(s^{-1}+\ldots s^{-2 k+3}\right)(x s)^{-(l-1)}=x^{-1} s^{-k+1}\left(s^{k-2}+\ldots+s^{-k+2}\right)(x s)^{-(l-1)}=x^{-1} s^{-k+1}[k-1](x
$$


Aplicando novamente o Lema 4.3.2 e usando o Teorema 3.3.2, temos que:

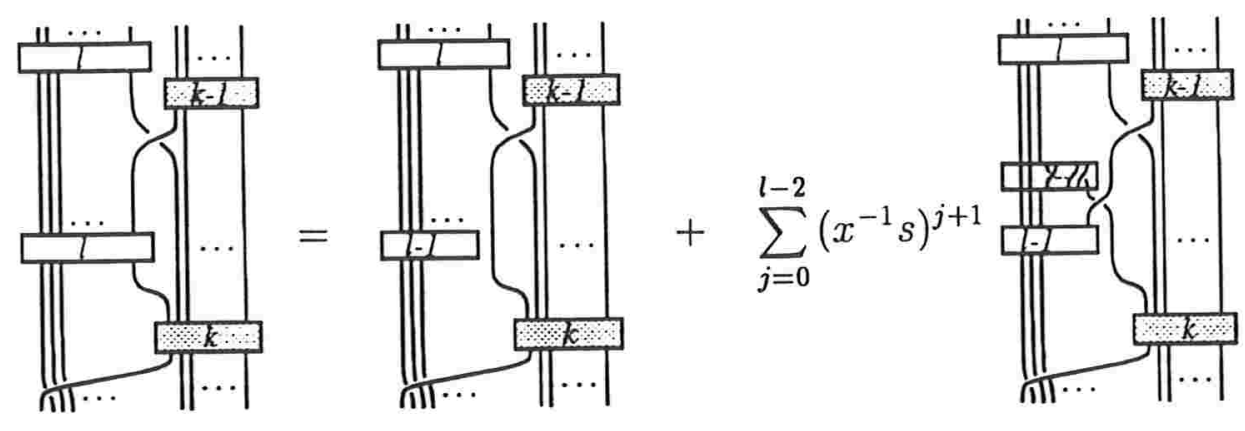

$$
=\left.\left(-x s^{-1}\right) \alpha_{1, l-1} \alpha_{k-1,1}\right|_{n \ldots 1} ^{l}
$$

Note que:

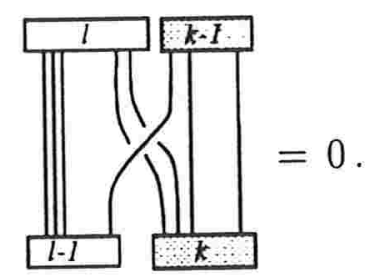

pois, como a $(l-1)$ linha e a $l$ linha são também adjacentes em $e_{k, 1}$, pelo Corolário 3.3.2, podemos fatorar $e_{1, l}$ e $e_{k, 1}$ de maneira a obter o termo:

$$
\left(\sigma_{l-1}-a\right)\left(\sigma_{l-1}-b\right)
$$

que é nulo em $H_{k+l-1}$.

Sendo assim: 


$$
e_{k, l}^{2}=\alpha_{k-1,1} \alpha_{1, l} e_{k, l}+s^{-k}[k-1](x s)^{-(l-1)}(x s)^{l-1} \alpha_{k-1,1} \alpha_{1, l-1}
$$

Aplicando a Proposição 4.3.2, temos:

$$
\begin{aligned}
\alpha_{k-1,1} & \alpha_{1, l}+s^{-k}[k-1] \alpha_{k-1,1} \alpha_{1, l-1} \\
& =\frac{[k-1] ![l-1] !}{s^{(k-1)(k-2) / 2}}\left(\frac{[l]}{s^{-l(l-1) / 2}}+\frac{s^{-k}[k-1]}{s^{-(l-1)(l-2) / 2}}\right) \\
& =\frac{[k-1] ![l-1] !}{s^{(k-1)(k-2) / 2}}\left(\frac{s^{l-1}[l]+s^{-k}[k-1]}{s^{-l(l-1) / 2} s^{l-1}}\right) \\
& =\frac{[k-1] ![l-1] !}{s^{(k-1)(k-2) / 2}}\left(\frac{s^{l-k}\left(s^{l-k-1}-s^{-l-k-1}+s^{k-1-l}-s^{-k+1-l}\right)}{s^{-l(l-1) / 2} s^{l-1}\left(s-s^{-1}\right)}\right) \\
& =\frac{[k-1] ![l-1] !}{s^{(k-1)(k-2) / 2} s^{-l(l-1) / 2}}\left(s^{-k+1} \frac{\left(s^{l+k-1}-s^{-l-k+1}\right)}{\left(s-s^{-1}\right)}\right) \\
& =\frac{[k-1] ![l-1] ![k+l-1]}{s^{(k-1)(k-2)+2(k-1)) / 2} s^{-l(l-1) / 2}} \\
& =\frac{[k-1] ![l-1] ![k+l-1]}{s^{k(k-1) / 2-l(l-1) / 2}}
\end{aligned}
$$

Nota: Vemos claramente com esse resultado, que o coeficiente $\alpha_{k, l}$ é especializado pela aplicação:

$$
\Gamma: H_{k+l-1} \rightarrow \mathbb{C} S_{n}
$$

no escalar $a_{\mu_{k, l}}$ (ver Teorema 3.2.5). Basta observar que quando $s \rightarrow 1$, temos $[i] \rightarrow i$ e que $\prod h l\left(\mu_{k, l}(i, j)\right)=(k+l+1)(k-1) !(l-1) !$

Convenção: Ao escrevermos $\chi(P)$, para $P \in C^{+}$, estamos nos referindo ao valor de $\chi(U * P)$, onde $U$ é diagrama do nó trivial sem "framing" (observando que o nó trivial com qualquer orientação representa a mesma classe de isotopia).

Queremos agora calcular o valor de $\chi\left(Q_{\lambda}\right)$, lembrando que $Q_{\lambda}$ é o fecho do idempotente $\left(1 / \alpha_{\lambda}\right) e_{\lambda}$. Observamos novamente que, apesar de estarmos trabalhando no anel $\Lambda=\mathbb{C} \llbracket h \rrbracket$, faremos frequentemente as devidas substituições após alguns cálculos. Além disso, vamos omitir, para "limparmos" a notação, a operação fecho nas equações deixando implícito que estamos calculando o HOMFLY do fecho do diagrama desenhado. 
Proposição 4.3.4 Quando $k \neq 0$, vale que:

$$
\chi\left(Q_{k, 1}\right)=\prod_{i=1}^{k} \frac{v^{-1} s^{-(i-1)}-v s^{i-1}}{s^{i}-s^{-i}}
$$

\section{Demonstração:}

Provaremos a igualdade fazendo indução em $k$. Para $k=1, Q_{1,1}=U$ e portanto:

$$
\chi\left(Q_{1,1}\right)=\delta=\frac{v^{-1}-v}{s-s^{-1}}
$$

Assumindo o resultado para $i<k$, basta mostrar que:

$$
\chi\left(Q_{k, 1}\right)=\left(\frac{v^{-1} s^{-k+1}-v s^{k-1}}{s^{k}-s^{-k}}\right) \chi\left(Q_{k-1,1}\right)
$$

De fato, lembrando que a aplicação $\chi: S\left(\mathbb{R}^{2}\right) \rightarrow \Lambda$ e a aplicação fecho são $\Lambda$-lineares, segue que:

$$
\chi\left(Q_{k, 1}\right)=\frac{1}{\alpha_{k, 1}} \chi\left(\widehat{e_{k, 1}}\right)=(*)
$$

Pelo Lema 4.3.2:

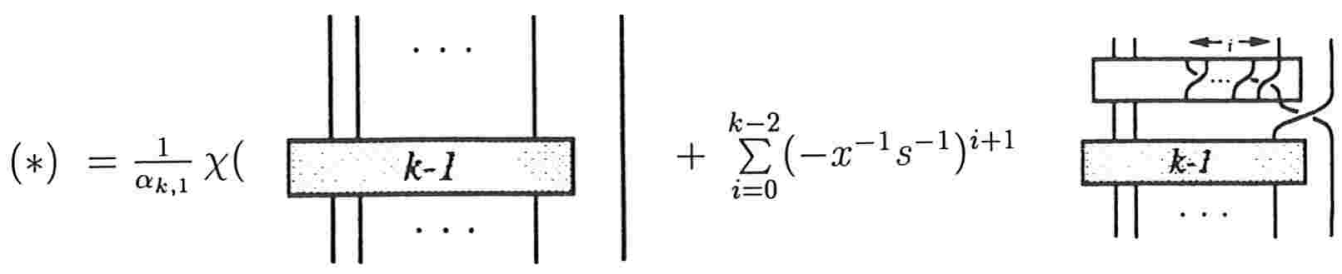

Pela relações $r 3, r 2$ e pelo Teorema 3.3.2:

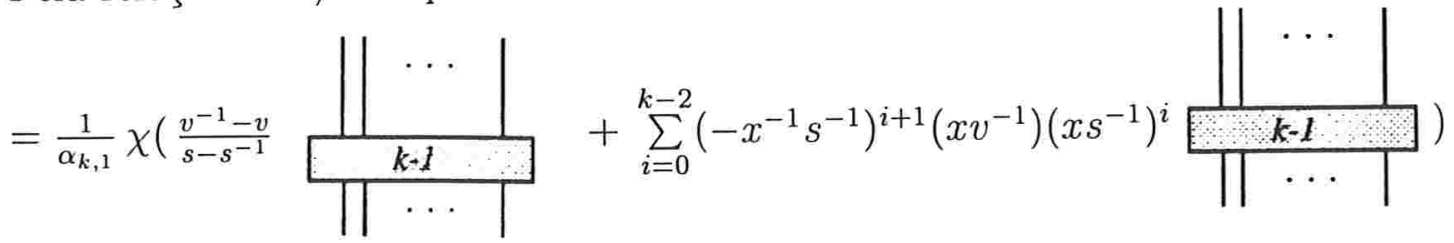

lembrando que $\omega_{i} b_{k-1}=\phi_{a}\left(\omega_{i}\right) b_{k-1}=a^{i} b_{k-1}$. 
Finalmente, pela Proposição 4.3.2 e observando que que $\sum_{i=0}^{k-2} s^{-2 i-2}=s^{-k}[k-1]$ temos:

$$
\begin{aligned}
& =\frac{\alpha_{k-1,1}}{\alpha_{k, 1}}\left(\frac{v^{-1}-v}{s-s^{-1}}-v^{-1}\left(\sum_{i=0}^{k-2} s^{-2 i-1}\right)\right) \chi\left(Q_{k-1,1}\right) \\
& =\frac{[k-1] !}{[k] !} \cdot \frac{s^{k(k-1) / 2}}{s^{(k-1)(k-2) / 2}}\left(\frac{v^{-1}-v}{s-s^{-1}}-v^{-1} s^{-k+1}[k-1]\right) \chi\left(Q_{k-1,1}\right) \\
& \frac{s^{k-1}}{[k]}\left(\frac{v^{-1}-v-v^{-1} s^{-k+1}\left(s^{k-1}-s^{-k+1}\right)}{s^{k}-s^{-k}}\right) \chi\left(Q_{k-1,1}\right) \\
& =\left(\frac{v^{-1} s^{-k+1}-v s^{k-1}}{s^{k}-s^{-k}}\right) \chi\left(Q_{k-1,1}\right) .
\end{aligned}
$$

como queríamos.

Definição 4.3.2 Denotamos por $\chi_{N}(L)$ o polinômio $\chi(L)$ calculado em $v=s^{-N}$ e $x=s^{-1 / N}$.

Corolário 4.3.2

$$
\chi_{N}\left(Q_{k, 1}\right)= \begin{cases}1 & , \quad p / k=N \\ 0 & , \quad p / k>N\end{cases}
$$

\section{Demonstração:}

Pela Proposição anterior temos:

$$
\chi\left(Q_{k, 1}\right)=\prod_{i=1}^{k} \frac{v^{-1} s^{-(i-1)}-v s^{i-1}}{s^{i}-s^{-i}}
$$

Quando $i=N+1$ e $v=s^{-N}$, temos:

$$
\frac{v^{-1} s^{-(i-1)}-v s^{i-1}}{s^{i}-s^{-i}}=\frac{s^{N} s^{-N}-s^{N} s^{N}}{s^{N+1}-s^{-(N+1)}}=0
$$


Logo, para $k>N, \chi\left(Q_{k, 1}\right)=0$. Para $k=N$ :

$$
\chi_{N}\left(Q_{N, 1}\right)=\frac{\left(v^{-1}-v\right)}{s-s^{-1}} \cdot \frac{\left(v^{-1} s^{-1}-v s\right)}{s^{2}-s^{-2}} \ldots \frac{\left(v^{-1} s^{-N+1}-v s^{N-1}\right)}{\left(s^{N}-s^{-N}\right)}
$$

Fazendo as substituições:

$$
\chi_{N}\left(Q_{N, 1}\right)=\frac{\left(s^{N}-s^{-N}\right)}{\left(s-s^{-1}\right)} \cdot \frac{\left(s^{N-1}-s^{-N+1}\right)}{\left(s^{2}-s^{-2}\right)} \ldots \frac{\left(s-s^{-1}\right)}{\left(s^{N}-s^{-N}\right)}=1
$$

Começam agora a aparecer algumas propriedades interessantes nas decorações pelos elementos $Q_{\lambda}$ que passam a justificar o interesse nesses elementos.

Proposição 4.3.5 Seja $L=L_{1} \cup L_{2} \cup \ldots \cup L_{n}$, um diagrama de link com $k$ componentes. Seja $L^{\prime}$ o satélite de $L$ obtido decorando $L_{i}$ com $Q_{\lambda_{i}}$ para $i<n$ e a $n$-ésima componente $L_{n}$ por $Q_{k, 1}$. Se $k>N$ então $\chi_{N}\left(L^{\prime}\right)=0$.

\section{Demonstração:}

$L$ pode ser representado claramente como o fecho de um elemento em $H_{1}$ na $n$-ésima componente:

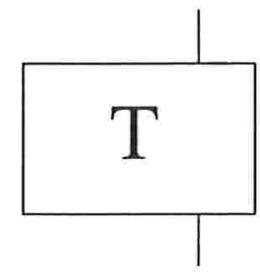

Logo $L^{\prime}$ pode ser visto como o fecho do elemento:

$$
\frac{1}{\alpha_{k, 1}}\left(T^{\prime} \cdot e_{k, 1}\right)
$$


Sendo assim, como $e_{k, 1}=b_{k}$, pelo Teorema 3.3.2:

$$
L^{\prime}=w\left(\frac{1}{\alpha_{k, 1}}\left(T^{\prime} \cdot e_{k, 1}\right)\right)=\frac{1}{\alpha_{k, 1}} \phi_{a}\left(T^{\prime}\right) \widehat{e_{k, 1}}
$$

Segue portanto que:

$$
\chi_{N}\left(L^{\prime}\right)=\frac{\phi_{a}\left(T^{\prime}\right)}{\alpha_{k, 1}} \chi_{N}\left(\widehat{e_{k, 1}}\right)=\phi_{a}\left(T^{\prime}\right) \chi_{N}\left(Q_{k, 1}\right)
$$

Logo, quando $k>0, \chi_{N}\left(L^{\prime}\right)=0$.

Lema 4.3.3 Sejam $L_{t}$ e $L_{b}$ diagramas de links que diferem localmente apenas por:
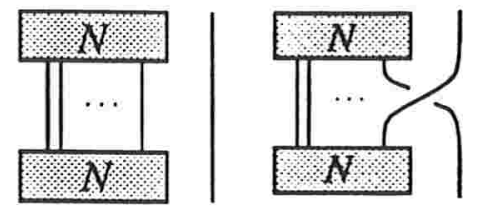

$L_{t}$

$L_{b}$

respectivamente. Então $\chi_{N}\left(L_{t}\right)=x^{-1} s^{-N}[N] \chi_{N}\left(L_{b}\right)$.

\section{Demonstração:}

Pelo Lema 4.3.2, temos:

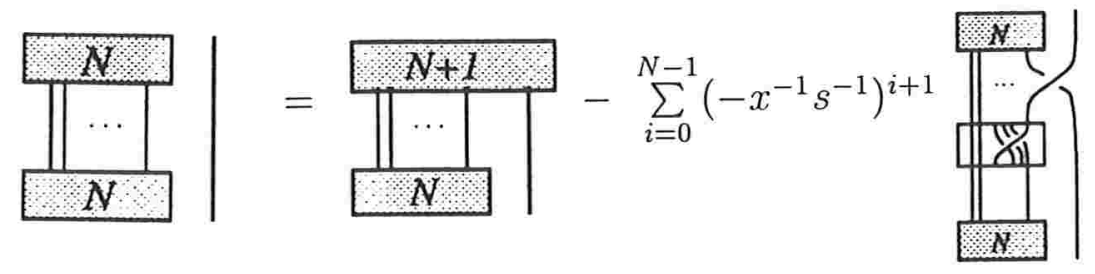


Observe que, pelo Teorema 3.3.2, temos:

$$
\begin{aligned}
\chi_{N}\left(\begin{array}{l|l|l|}
N+1 \\
\mid
\end{array}\right. & =\phi_{a}\left(b_{N}\right) \chi_{N}\left(\widehat{b_{N}}\right) \\
& =\phi_{a}\left(b_{N}\right) \alpha_{N+1,1} \chi_{N}\left(Q_{N+1,1}\right)=0
\end{aligned}
$$

pelo Lema 4.3.4.

Logo, novamente pelo Teorema 3.3.2:

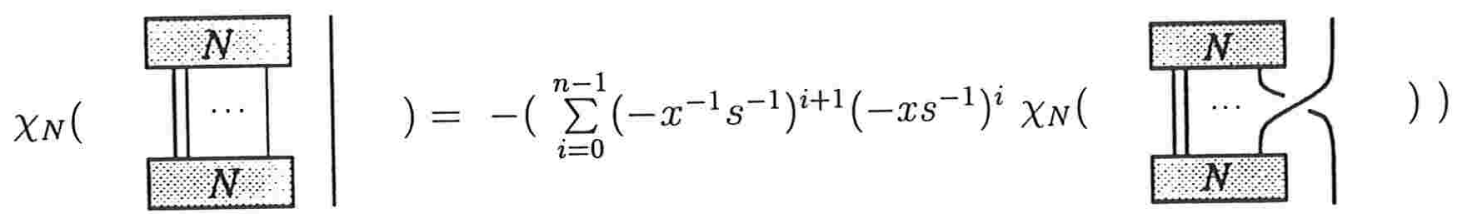

$=x^{-1}\left(\sum_{i=0}^{N-1} s^{-2 i-1}\right) \chi_{N}($

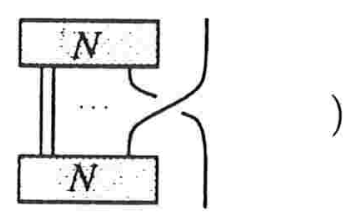

$=x^{-1} s^{-N}[N] \chi_{N}($

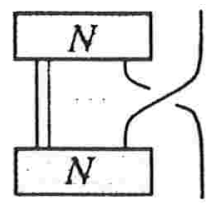


Lema 4.3.4 Sejam $L_{t}$ e $L_{b}$ dois diagramas de links que diferem localmente somente por:
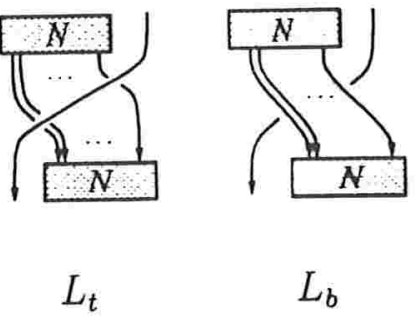

respectivamente. Então $\chi_{N}\left(L_{t}\right)=\chi_{N}\left(L_{b}\right)$.

\section{Demonstração:}

Para simplificar a notação, sempre vamos considerar os diagramas abaixo como um "retrato local" de diagramas fechados. Pelo Lema anterior:
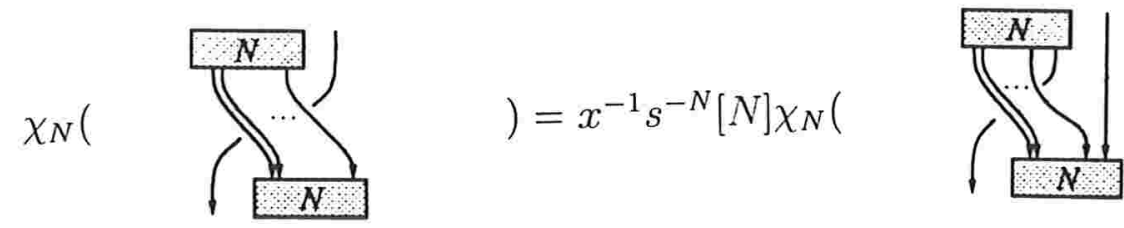

Aplicando o Teorema 3.3.2:

$$
=x^{-1} s^{-N}[N]\left(-x s^{-1}\right)^{-N+1} \chi_{N}(
$$

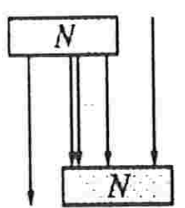


Pelo mesmo resultado temos:

$=x^{-1} s^{-N}[N]\left(-x s^{-1}\right)^{-2(N-1)} \chi_{N}($

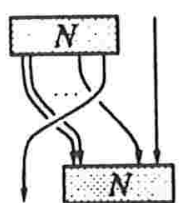

E pela relação $\boldsymbol{r} 1$ :

$=x^{-1} s^{-N}[N]\left(-x s^{-1}\right)^{-2(N-1)} \chi_{N}\left(x^{-2}\right.$

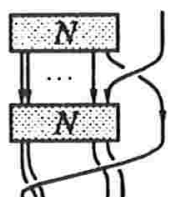

$-x^{-1}\left(s-s^{-1}\right)$

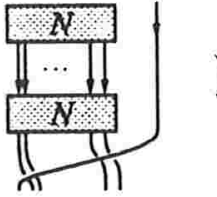

Pelo Lema anterior:

$$
\begin{aligned}
& =x^{-1} s^{-N}[N] a^{-2(N-1)}\left(x^{-2} x s^{N}[N]^{-1}-x^{-1}\left(s-s^{-1}\right)\right) \chi_{N}\left(L_{t}\right) \\
& =\left(\frac{x^{-3} s^{-N}[N] \cdot\left(-x s^{-1}\right)^{-2(n-1)}}{x^{-1} s^{-N}[N]}-x^{-2} s^{-N}[N]\left(-x s^{1}\right)^{-2(N-1)}\left(s-s^{-1}\right)\right) \chi_{N}\left(L_{t}\right) \\
& =\left(x^{-2 N} s^{2 N-2}-x^{-2 N} s^{-N}\left(s^{N}-s^{-N}\right) s^{2 N-2}\right) \chi_{N}\left(L_{t}\right) \\
& =\left(x^{-2 N} s^{2 N-2}-x^{-2 N} s^{N-2}\left(s^{N}-s^{-N}\right)\right) \chi_{N}\left(L_{t}\right) \\
& =x^{-2 N}\left(s^{2 N-2}-s^{2 N-2}+s^{-2}\right) \chi_{N}\left(L_{t}\right) \\
& =x^{-2 N} s^{-2} \chi_{N}\left(L_{t}\right)
\end{aligned}
$$

Fazendo a substituição $x=s^{-1 / N}$ temos a igualdade desejada. 
Proposição 4.3.6 Seja $L=L_{1} \cup L_{2} \cup \ldots \cup L_{n}$ um diagrama de link com $n$ componentes. Seja $L^{\prime}$ o diagrama obtido removendo-se a $n$-ésima componente $L_{n}$. Seja $\widetilde{L^{\prime}}$ obtido decorando-se cada componente de $L$ por $Q_{\lambda_{i}}$, e $\widetilde{L}$ obtido da mesma forma adicionando-se apenas a decoração $L_{n} * Q_{N, 1}$. Entãoo $\chi_{N}(\widetilde{L})=\chi_{N}\left(\widetilde{L^{\prime}}\right)$.

\section{Demonstração:}

Observamos primeiramente que $Q_{\lambda_{N, 1}}=\left(1 / \alpha_{N, 1}\right) \widehat{e_{N, 1}}$, onde $e_{N, 1}=b_{N}$, e portanto, localmente, podemos considerar cada cruzamento da componente $L_{n} * Q_{\lambda_{N, 1}}$ com outra componente $L_{i} * Q_{\lambda_{i}}$ com seguinte forma:

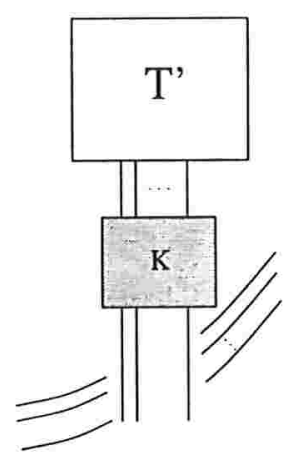

Suponha que ocorra o primeiro caso (o outro é completamente análogo). Vamos mostrar que podemos, para efeito de cálculo de $\chi_{N}$, desconectar a $n$-ésima componente das outras.

De fato, lembrando que $b_{N}^{2}=\phi_{a}\left(b_{N}\right) b_{N}$, onde $\phi_{a}\left(b_{N}\right)=\alpha_{N, 1}$, escalar que já provamos ser inversível, o diagrama acima é o mesmo, a menos de um escalar que:

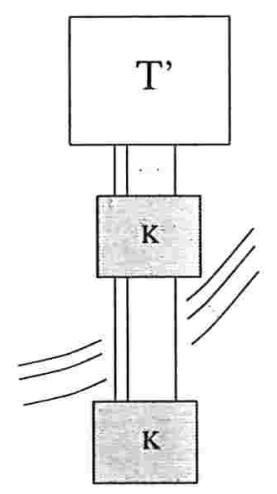


Pelo Lema anterior, o valor de $\chi_{N}$ desse diagrama é igual ao do diagrama:

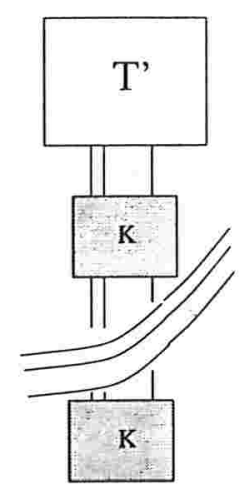

Logo, continuando esse processo (o número de cruzamentos entre a n-ésima componente do nó decorado e as demais é obviamente finito), para efeito de cálculo de $\chi_{N}$, podemos considerar a $n$-ésima componente desligada do resto do diagrama. Da mesma forma, podemos considerá-la como o nó trivial decorado por $Q_{N, 1}$, usando os mesmos argumentos.

Obtemos portanto a seguinte igualdade:

$$
\chi_{N}(\widetilde{L})=\chi_{N}\left(\widetilde{L^{\prime}} \cup Q_{N, 1}\right)
$$

Pela propriedade multiplicativa do polinômio HOMFLY e pelo Corolário 4.3.4, temos:

$$
\chi_{N}(\widetilde{L})=\chi_{N}\left(\widetilde{L^{\prime}}\right) \cdot \chi_{N}\left(Q_{N, 1}\right)=\chi_{N}\left(\widetilde{L^{\prime}}\right)
$$

como queríamos.

Fazendo demonstração análoga a da Proposição 4.3.3, temos o seguinte resultado:

Lema 4.3.5 ([Anna], pg.109) $s^{l}[l] \widehat{e_{k+1, l}}+s^{-k}[k] \widehat{e_{k, l+1}}=s^{l-k}[l+k] \widehat{e_{1, l}} \cdot \widehat{e_{k, 1}}$ 
A proposição a seguir, mostra uma relação entre os elementos $Q_{k, l}$ a qual é extremamente semelhante com o produto dos diagramas de Young $c_{k}$ e $d_{l}$. Veremos posteriormente que esse fato não é uma mera coincidência.

Proposição $4.3 .7 \quad Q_{k+1, l}+Q_{k, l+1}=Q_{k, 1} Q_{1, l}$

\section{Demonstração:}

Do Lema anterior temos:

$$
s^{l}[l] \alpha_{k+1, l} Q_{k+1, l}+s^{-k}[k] \alpha_{k, l+1} Q_{k, l+1}=s^{l-k}[l+k] \alpha_{1, l} \alpha_{k, 1} Q_{1, l} Q_{k, 1}
$$

Substituindo os valores encontrados na Proposição 4.3.3, temos:

$$
\frac{s^{l}[l] \alpha_{k+1, l}}{s^{l-k}[l+k] \alpha_{k, 1} \alpha_{1, l}}=\frac{s^{l}[l][k+l][k] ![l-1] ! s^{k(k-1) / 2} s^{-l(l-1) / 2}}{s^{l-k}[l+k] s^{k(k+1) / 2} s^{-l(l-1) / 2}[k] ![l] !}=\frac{s^{l}}{s^{l-k} s^{k}}=1
$$

Analogamente:

$$
\frac{s^{-k} \alpha_{k, l+1}}{s^{l-k}[l+k] \alpha_{k, 1} \alpha_{1, l}}=\frac{s^{-k}[k][k+l][k-1] ![l] ! s^{k(k-1 / 2)} s^{-l(l-1) / 2}}{s^{l-k}[l+k] s^{k(k-1) / 2} s^{-l(l+1) / 2}[k] ![l] !}=\frac{s^{-k}}{s^{l-k} s^{l}}=1
$$

Logo vale que $Q_{k+1, l}+Q_{k, l+1}=Q_{k, 1} Q_{1, l}$.

Novamente usaremos séries formais de potências (nesse caso com coeficientes em $C^{+}$) a fim de obtermos algumas igualdades úteis.

Proposição 4.3.8 Sejam $Q_{C}(X)$ e $Q_{D}(X)$ séries formais de potências com coeficientes no anel $C^{+}$dadas por:

$$
Q_{C}(X)=\sum_{k=0}^{\infty}(-1)^{k} Q_{k, 1} X^{k}
$$




$$
Q_{D}(X)=\sum_{l=0}^{\infty}(-1)^{k} Q_{1, l} X^{l}
$$

Então $Q_{C} X Q_{D}(X)=1$.

\section{Demonstração:}

O coeficiente de $X^{0}$ na série produto é dado por $Q_{0,1} Q_{1,0}$, que é o diagrama vazio, o qual por sua vez representa a identidade em $C^{+}$.

Logo basta mostrar que o coeficiente de $X^{m}$ é zero para qualquer $m>0$. Usando a Proposição anterior, temos que:

$$
\begin{aligned}
& \sum_{k=0}^{m}(-1)^{k} Q_{k, 1} Q_{1, m-k}=Q_{1, m}+(-1)^{m} Q_{m, 1}+\sum_{k=1}^{m-1}(-1)^{k}\left(Q_{k+1, m-k}+Q_{k, m-k+1}\right) \\
& =Q_{1, m}+(-1)^{m} Q_{m, 1}+\sum_{k=1}^{m-1}(-1)^{k} Q_{k+1, m-k}+\sum_{k=0}^{m-2}(-1)^{k+1} Q_{k+1, m-k} \\
& =Q_{1, m}+(-1)^{m} Q_{m, 1}+(-1)^{m-1} Q_{m, 1}+(-1) Q_{1, m}+\sum_{k=1}^{m-2}(-1)^{k}\left(Q_{k+1, m-k}-Q_{k+1, m-k}\right) \\
& =0
\end{aligned}
$$

Lema 4.3.6 Podemos expressar $\chi\left(Q_{C}(X)\right)$ como um produto formal infinito de funções racionais em $X$ com coeficientes no anel $\Lambda$ :

$$
\chi\left(Q_{C}(X)\right)=\prod_{k=0}^{\infty} \frac{1-v s^{2 k+1} X}{1-v^{-1} s^{2 k+1} X}
$$

\section{Demonstração:}

Seja $P(X)=\prod_{k=0}^{\infty} \frac{1-v s^{2 k+1}}{1-v^{-1} s^{2 k+1} X}$. Como série formal de potências podemos escrever $P(X)$ na forma:

$$
P(X)=\sum_{r=0}^{\infty} p_{r} X^{r}
$$


Note que $P(X)=\frac{1-v s X}{1-v^{-1} s X} P\left(s^{2} X\right)$ e portanto:

$$
\left(1-v^{-1} s X\right) P(X)=(1-v s X) P\left(s^{2} X\right)
$$

Logo:

$$
\sum_{r=0}^{\infty}\left(1-v^{-1} s X\right) p_{r} X^{r}=\sum_{r=0}^{\infty}(1-v s X) s^{2 r} p_{r} X^{r}
$$

De onde tiramos que:

$$
p_{r+1}-v^{-1} s p_{r}=s^{2 r+2} p_{r+1}-v s^{2 r+1} p_{r}
$$

E portanto:

$$
p_{r+1}=\frac{v^{-1} s-v s^{2 r+1}}{1-s^{2 r+2}} p_{r}=\frac{s^{r+1}\left(v s^{r}-v^{-1} s^{-r}\right)}{s^{r+1}\left(s^{r+1}-s^{-r-1}\right)} p_{r}=\frac{\left(v s^{r}-v^{-1} s^{-r}\right)}{\left(s^{r+1}-s^{-(r+1)}\right)} p_{r}
$$

O valor de $p_{0}$ pode ser obtido fazendo $x=0$, o que implica em $p_{o}=P(0)=1$. Por outro lado, para $r>0$ temos:

$$
p_{r}=\prod_{i=0}^{r} \frac{v s^{i-1}-v^{-1} s^{-(i-1)}}{s^{i}-s^{-i}}=\prod_{i=0}^{r} \frac{(-1) v^{-1} s^{-(i-1)-v s^{i-1}}}{s^{i}-s^{-i}}=(-1)^{r} \chi\left(Q_{r, 1}\right)
$$

pela Proposição 4.3.4.

Proposição $4.3 .9 \chi\left(Q_{1, l}\right)=\prod_{i=1}^{l} \frac{v^{-1} s^{i-1}-v s^{-(i-1)}}{s^{i}-s^{-i}}$.

\section{Demonstração:}

Pela Proposição 4.3.8, temos que:

$$
\chi\left(Q_{C} Q_{D}\right)=\chi\left(Q_{C}\right) \chi\left(Q_{D}\right)=1
$$

Logo, pelo Lema anterior:

$$
\chi\left(Q_{D}\right)=\prod_{k=0}^{\infty} \frac{1-v^{-1} s^{2 k+1} X}{1-v s^{2 k+1} X}
$$


Sendo assim, os coeficientes de $\chi\left(Q_{D}\right)$ como série formal são obtidos trocando-se $v$ por $v^{-1}$ (e vice versa) nos coeficientes de $\chi\left(Q_{C}\right)$. Logo,

$$
\chi\left(Q_{1, l}\right)=\prod_{i=1}^{l} \frac{v^{-1} s^{i-1}-v s^{-(i-1)}}{s^{i}-s^{-i}}
$$

Proposição 4.3.10 $\chi_{N}\left(Q_{k, 1}\right)=\chi_{N}\left(Q_{N-k, 1}\right)$.

\section{Demonstração:}

Quando $k \neq 0$, pela Proposição 4.3.4, temos:

$$
\chi\left(Q_{N-k, 1}\right)=\prod_{j=1}^{N-k} \frac{v^{-1} s^{-(j-1)}-v s^{j-1}}{s^{j}-s^{-j}}
$$

Fazendo $v=s^{-N}$, temos:

$$
\begin{aligned}
& \chi_{N}\left(Q_{N-k, 1}\right)=\prod_{j=1}^{N-k} \frac{s^{N} s^{-j+1}-s^{-N} s^{j-1}}{s^{j}-s^{-j}} \\
& =\prod_{j=1}^{N-k} \frac{s^{N-j+1}-s^{-N+j-1}}{s^{j}-s^{-j}} \\
& =\frac{[N]}{[1]} \cdot \frac{[N-1]}{[2]} \ldots \frac{[K+1]}{[N-K]} \\
& =\frac{[N] !}{[k] ![N-k] !} \\
& =\frac{[N]}{[1]} \cdot \frac{[N-1]}{[2]} \ldots \frac{[N-k+1]}{[k]}
\end{aligned}
$$

Por outro lado, novamente pela Proposição 4.3.4 e após fazermos a substituição $v=s^{-N}$ :

$$
\chi_{N}\left(Q_{k, 1}\right)=\prod_{j=1}^{k} \frac{s^{N-j+1}-s^{-N+j-1}}{s^{j}-s^{-j}}
$$




$$
\begin{aligned}
& =\frac{\left(s^{N}-s^{-N}\right)}{\left(s-s^{-1}\right)} \frac{s^{N-1}-s^{-N+1}}{s^{2}-s^{-2}} \ldots \frac{\left(s^{N-k+1}-s^{-N+k-1}\right)}{\left(s^{k}-s^{-k}\right)} \\
& =\frac{[N]}{[1]} \cdot \frac{[N-1]}{[2]} \ldots \frac{[N-k+1]}{[k]}
\end{aligned}
$$

E portanto, temos que $\chi_{N}\left(Q_{k, 1}\right)=\chi_{N}\left(Q_{N-k, 1}\right)$, quando $k \neq 0$.

Quando $k=0, Q_{0,1}$ é o diagrama vazio e portanto $\chi_{N}\left(Q_{0,1}\right)=1$. Por outro lado, $\chi_{N}\left(Q_{N, 1}\right)=1$ pelo Corolário 4.3.2 e portanto vale a igualdade para todo $k \geq 0$, como queríamos.

Provaremos agora, sempre visando o nosso principal objetivo desse capítulo que é explicitar o isomorfismo entre $Y$ e $C^{+}$, mais um propriedade importante dos elementos $Q_{\lambda}$. Antes citaremos algumas proposições concernentes à teoria de módulos:

Proposição 4.3.11 [COHN] Seja $R$ um anel com unidade. Seja $M=\bigoplus_{j \in J} M_{j}$, soma direta de $R$-módulos livres $M_{j}$ com base $B_{j}$ para todo $j$. Então, $M$ é $R$-módulo livre com base $\bigcup_{j \in J} B_{j}$.

Proposição 4.3.12 [COHN] Seja $R$ anel comutativo e $M$ um $R$-módulo livre. Então toda base de $M$ tem a mesma cardinalidade.

Corolário 4.3.3 Sejam $R$ anel comutativo, $M$ um $R$-módulo e $B=\left\{m_{1}, \ldots, m_{k}\right\}$ uma base de $M$. Seja $D \subseteq M$ um conjunto L.I. com $k$ elementos. Então, $D$ é base de $M$.

Proposição 4.3.13 $O$ conjunto $\left\{Q_{\lambda}\right\}$, onde $\lambda$ percorre o conjunto dos diagramas de Young, é uma base para o $\Lambda$-módulo livre $C^{+}$.

\section{Demonstração:}

Vamos mostrar que $\left\{Q_{\lambda} /|\lambda|=n\right\}$ é base do $\Lambda$-submódulo $C^{(n)}$, mostrando que todo $c \in C^{+}$pode ser escrito como combinação linear desses elementos. Isso basta, pois a dimensão de $C^{(n)}$ é igual ao número de partições de $n$ e portanto 
igual ao número de diagramas de Young com $n$ células. Pelo Corolário 4.3.3, temos que $\left\{Q_{\lambda} /|\lambda|=n\right\}$ é base do $\Lambda$-submódulo $C^{(n)}$. Isso feito, pela Proposição 4.3 .11 teremos mostrado que $\left\{Q_{\lambda}\right\}$ é base de $C^{+}$.

De fato, seja $c \in C^{(n)}$. Temos que existe um $h \in H_{n}$ tal que $\widehat{h}=c$. Pela Proposição 3.5.1:

$$
H_{n}=\sum_{|\lambda|=n} H_{n} e_{\lambda} H_{n}
$$

e portanto $h$ pode ser escrito na forma $h=\sum_{|\lambda|=n} h_{\lambda}^{1} e_{\lambda} h_{\lambda}^{2}$, onde $h_{\lambda}^{i} \in H_{n}$.

Por outro lado, como estamos trabalhando no anel e como $e_{\lambda}^{2}=\alpha_{\lambda} e_{\lambda}$, onde $\alpha_{\lambda}$ é um escalar inversível temos que:

$$
\widehat{h}=\sum_{|\lambda|=n} \frac{1}{\alpha_{\lambda}} \widehat{h_{\lambda}^{1}} \widehat{e_{\lambda}^{2}} h_{\lambda}^{2}=\sum_{|\lambda|=n} \frac{1}{\alpha_{\lambda}} e_{\lambda} \widehat{h_{\lambda}} e_{\lambda}
$$

onde $h_{\lambda}=h_{\lambda}^{2} h_{\lambda}^{1}$. Sendo assim:

$$
c=\widehat{h}=\sum_{|\lambda|=n} \frac{1}{\alpha_{\lambda}} e_{\lambda} \widehat{h_{\lambda}} e_{\lambda}(*)
$$

Lembrando que $e_{\lambda}=E_{\lambda}(a) \omega_{\pi_{\lambda}} E_{\lambda^{v}}(b) \bar{\omega}_{\pi_{\lambda^{v}}}$, temos:

$$
(*)=\sum_{|\lambda|=n} \frac{1}{\alpha_{\lambda}} E_{\lambda}(a)\left(\omega_{\pi_{\lambda}} E_{\lambda^{v}}(b) \bar{\omega}_{\pi_{\lambda v}} h_{\lambda} E_{\lambda}(a) \omega_{\pi_{\lambda}}\right) E_{\lambda^{v}}(b) \bar{\omega}_{\pi_{\lambda^{v}}}
$$

Vamos chamar o elemento $\left(\omega_{\pi_{\lambda}} E_{\lambda^{v}}(b) \bar{\omega}_{\pi_{\lambda^{v}}} h_{\lambda} E_{\lambda}(a) \omega_{\pi_{\lambda}}\right)$ de $\bar{h}_{\lambda}$.

Lembrando que $\left\{\omega_{\pi} / \pi \in S_{n}\right\}$ é uma base de $H_{n}$ visto como um $\Lambda$-módulo, temos que:

$$
e_{\lambda} h e_{\lambda}=E_{\lambda}(a) \bar{h}_{\lambda} E_{\lambda^{v}}(b) \bar{\omega}_{\pi_{\lambda^{v}}}=E_{\lambda}(a)\left(\sum_{\tau \in S_{n}} \gamma_{\tau} \omega_{\tau}\right) E_{\lambda^{v}}(b) \bar{\omega}_{\pi_{\lambda v}}
$$

Pelo Lema 3.3.4, se $\tau$ não separa $\lambda$ e $\lambda^{v}$ então:

$$
E_{\lambda}(a) \omega_{\tau} E_{\lambda^{v}}(b)=0 .
$$

Seja $S_{\text {sep }}=\left\{\tau \in S_{n} / \tau\right.$ separa $\left.\lambda \mathrm{e} \lambda^{v}\right\}$ Quando $\rho \in S_{\text {sep }}$, pelo Lema 3.3.5, existem $\rho_{1} \in R(\lambda)$ e $\rho_{2} \in R\left(\lambda^{v}\right)$ tais que:

$$
\omega_{\tau}=\omega_{\rho_{1}}^{\tau} \omega_{\pi_{\lambda}} \omega_{\rho_{2}}^{\tau}
$$


Substituindo em $(* *)$ :

$$
e_{\lambda} h e_{\lambda}=\sum_{\tau \in S_{\text {sep }}} \gamma_{\tau} E_{\lambda}(a) \omega_{\rho_{1}}^{\tau} \omega_{\pi_{\lambda}} \omega_{\rho_{2}}^{\tau} E_{\lambda^{v}}(b) \bar{\omega}_{\pi_{\lambda^{v}}}
$$

E pelo Lema 3.3.2, como $\rho_{1} \in R(\lambda)$ e $\rho_{2} \in R\left(\lambda^{v}\right)$ temos:

$$
e_{\lambda} h e_{\lambda}=\sum_{\tau \in S_{\text {sep }}} \beta(\tau) \gamma_{\tau} e_{\lambda}
$$

Sendo assim:

$$
c=\sum_{|\lambda|=n} \frac{1}{\alpha_{\lambda}}\left(\sum_{\tau \in S_{\text {sep }}} \gamma_{\tau} \beta(\tau)\right) \widehat{e_{\lambda}}=\sum_{|\lambda|=n} \xi_{\lambda} Q_{\lambda},
$$

onde $\xi_{\lambda}=\sum_{\tau \in S_{\text {sep }}} \gamma_{\tau} \beta(\tau) \in . \perp$ como queríamos.

Obs: A partir de agora não faremos mais distinção entre $Y$ e $R_{\infty}$.

Para o próximo resultado, usaremos a relação (citada no cap.1) entre polinômio HOMFLY, $\chi(L)$ e o o polinômio de Conway, $\nabla(L)$, como também de alguns resultados preliminares.

Proposição 4.3.14 O homomorfismo de álgebras $\theta: R_{\infty} \rightarrow C^{+}$definido por:

$$
\theta\left(c_{i}\right)=Q_{c_{i}}
$$

é um isomorfismo de álgebras.

\section{Demonstração:}

Denotemos, por hora, $Q_{c_{i}}=Q_{i}$. Sabemos que $R_{\infty}$ é uma álgebra livre gerada pelos $\left\{c_{i} / i=\mathbb{N}\right\}$. Vamos mostrar que os elementos $Q_{i}{ }^{\prime} s$ geram $C^{+}$, e portanto $\theta$ é sobrejetora, e de forma livre, o que implica que $\theta$ é injetora (basta ver que a existência de dois elementos distintos $m_{1}$ e $m_{2}$ tais que $\theta\left(m_{1}\right)=\theta\left(m_{2}\right)$, implica na existência de um polinômio com coeficientes não todos nulos nos $Q_{i}{ }^{\prime} s$, mas que é nulo na álgebra $\left.C^{+}\right)$. 
Lembremos primeiramente que (Teorema 2.4.1) os elementos $\varphi_{m}, m \in \mathbb{N}$, onde $\varphi_{m}$ é o fecho da trança $\sigma_{m-1} \sigma_{m-2} \cdots \sigma_{1}$, geram $C^{+}$livremente como álgebra.

Por indução em $n$, vamos mostrar que $\varphi_{n}$ pode ser escrito como uma expressão polinomial nos $Q_{i}{ }^{\prime} s$ com coeficientes em $\Lambda$. Para $n=1, Q_{1}=\varphi_{1}$, e portanto nada temos a demonstrar.

Assumimos então que para todo $\varphi_{m}$, com $m<n$, temos uma expressão polinomial em termos $\operatorname{dos} Q_{i}{ }^{\prime} s, i \leq m$.

Temos que $e_{n} \in H_{n}$ implica que $\frac{1}{\alpha_{n}} \widehat{e_{n}} \in C^{(n)}$. Logo, $Q_{n}$ pode ser expresso linearmente pelos fechos dos monômios de peso $n$ em $\varphi_{m}$, os quais são indexados pelas partições de $n$. Sendo assim:

$$
Q_{n}=\beta_{n} \varphi_{n}+\sum_{\lambda=\left(\lambda_{1}, \ldots, \lambda_{n}\right),|\lambda|=n} \beta_{\lambda} \varphi_{\lambda_{1}} \varphi_{\lambda_{2}} \ldots \varphi_{\lambda_{k}}
$$

onde $\beta_{n}, \beta_{\lambda} \in \Lambda$.

Provaremos agora que $\beta_{n}$ é inversível em $\Lambda$. Isso feito, usando a hipótese de indução, concluimos que $\varphi_{n}$ pode ser escrito como um polinômio nos $Q_{i}{ }^{\prime} s$, para $i \leq n$.

Pela observação já feita no Capítulo 1 , o polinômio de Conway $\nabla(L)$ pode ser calculado a partir do HOMFLY colocando-se $x=v=1$. Pela Proposição 1.3.1, esse polinômio se anula quando $L$ é um "split link", ou melhor, um link que possue componentes deconectadas.

Note que todos os termos do lado direito da igualdade $(*)$, exceto $\varphi_{n}$, são "split links". Além disso, $\varphi_{n}$ é equivalente (em termos de isotopia ambiente) ao nó trivial. Sendo assim, temos:

$$
\nabla\left(Q_{n}\right)=\nabla\left(\beta_{n} \varphi_{n}\right)=\beta_{n}^{\prime}
$$

onde $\beta_{n}^{\prime}$ é o coeficiente $\beta_{n}$ calculado em $x=v=1$.

Lembrando que fizemos as substituições $x=s^{-1 / N}$ e $v=s^{-N}$, esses elementos como séries formais de potências em $h$, possuem termo constante igual a 1 . Logo o termo constante de $\beta_{n}$ é igual ao de $\beta_{n}^{\prime}$.

Pela Proposição 3.5.1, mostrando que termo constante de $\beta_{n}^{\prime}=\nabla\left(Q_{n}\right)$ é não nulo, mostramos que $\beta_{n}$ é inversível em $\Lambda$. Voltando a primeira expressão de $Q_{n}$, temos:

$$
Q_{n}=\frac{1}{\alpha_{n}} \widehat{e_{n}}=\frac{1}{\alpha_{n}} \widehat{b_{n}}
$$


onde $b_{n}=\sum_{\pi \in S_{n}}\left(-x^{-1} s^{-1}\right)^{l(\pi)} \omega_{\pi}$. Queremos ver quais os termos da expressão de $Q_{n}$ que contribuem ao termo constante de $\nabla\left(Q_{n}\right)$ como série de potências em $h$.

Pela Proposição 1.3.2, e como $z=e^{h / 2}-e^{-h / 2}$, os únicos termos em $b_{n}$ que contribuem com o termo constante são as tranças $\omega_{\pi}$ cujos fechos são nós, as quais pela Proposição 1.4.1, são tais que $\pi$ é $n$-ciclo.

O coeficiente de $\omega_{\pi}$ em $Q_{n}$ é $\left(-x^{-1} s^{-1}\right)^{l(\pi)}$. Por outro lado, como $\pi$ é um $n$-ciclo, temos que:

$$
l(\pi) \equiv(n-1) \bmod (2)
$$

e portanto o termo constante de $\left(-x^{-1} s^{-1}\right)^{l(\pi)}$ como série de potências em $h$ é $(-1)^{n-1}$.

Pela Proposição 1.4 .2 e por existirem $(n-1)$ ! permutações que são $n$-ciclos em $S_{n}$, temos que o termo constante de $Q_{n}$ como série de potências em $h$ é:

$$
g\left(\frac{1}{\alpha_{n}}\right)(n-1) !(-1)^{n-1}
$$

e portanto não nulo.

Provamos então que o conjunto $\left\{Q_{i} / i \in \mathbb{N}\right\}$ gera $C^{+}$como $\Lambda$-álgebra e portanto os monômios nesses elementos geram $C^{+}$como $\Lambda$-módulo. Vamos mostrar que na verdade eles formam uma base para esse espaço, provando assim que os $Q_{i}{ }^{\prime} s$ geram a álgebra $C^{+}$de forma livre.

$$
\begin{aligned}
& \text { Seja } \mathcal{A}=\bigcup_{n \in \mathbb{N}} A_{n} \text {, onde: } \\
& \qquad A_{n}=\left\{Q_{i_{1}}^{j_{1}} Q_{i_{2}}^{j_{2}} \ldots Q_{i_{p}}^{j_{p}} / i_{1} \geq i_{2} \geq \ldots \geq i_{p} \text { e } \sum_{k=0}^{p} i_{k} j_{k}=n\right\} .
\end{aligned}
$$

Observamos que, pela comutatividade de $C^{+}$, podemos supor $i_{1} \geq \ldots \geq i_{p}$ e usando o mesmo raciocínio feito no Corolário 2.4.1, concluimos que $A_{n}$ é indexado pelos diagramas de Young.

Por outro lado, temos $A_{n} \subseteq C^{(n)}$ gerando-o como $\Lambda$-módulo. Como a dimensão de $C^{(n)}$ é o número de partições de $n$ e portanto de diagramas de Young com $n$ células, pelo Corolário 4.3.3, $A_{n}$ é base desse espaço. Pela Corolário 2.4.1, como $C^{+}=\bigoplus_{n \in \mathbb{N}} C^{(n)}, \mathcal{A}$ é base de $C^{+}$como $\Lambda$-módulo.

Proposição 4.3.15 Para todo $l \geq 1$, vale que $\theta\left(d_{l}\right)=Q_{1, l}$. 


\section{Demonstração:}

Vamos demonstrar o resultado fazendo indução em $l$. Observe que para $l=1$, $d_{1}=c_{1}$ e portanto $\theta\left(d_{1}\right)=Q_{1,1}$. Assumiremos o resultado válido para todo $d_{l}$, tal que $l<m$.

Pela Proposição 4.2.4, $\sum_{k=0}^{m}(-1)^{k} c_{k} d_{m-k}=0$, para todo $m \geq 1$. Como $\theta$ é isomorfismo de álgebras, temos:

$$
\sum_{k=0}^{m}(-1)^{k} \theta\left(c_{k}\right) \theta\left(d_{m-k}\right)=0
$$

Temos também pela Proposição 4.3.8 que:

$$
\sum_{k=0}^{m} Q_{k, 1} Q_{1, m-k}=0
$$

Pela hipótese de indução, temos:

$$
\sum_{k=0}^{m}(-1)^{k} Q_{k, 1} Q_{1, m-k}=Q_{m}+\sum_{k=1}^{m}(-1)^{k} \theta\left(c_{k}\right) \theta\left(d_{m-k}\right)
$$

De 4.1 e 4.2 obtemos:

$$
0=Q_{m}+\sum_{k=1}^{m}(-1)^{k} \theta\left(c_{k}\right) \theta\left(d_{m-k}\right)-\sum_{k=0}^{m}(-1)^{k} \theta\left(c_{k}\right) \theta\left(d_{m-k}\right)
$$

E portanto:

$$
0=Q_{m}-\theta\left(d_{m}\right) \Longrightarrow \theta\left(d_{m}\right)=Q_{m}
$$

Proposição 4.3.16 $\theta\left(\mu_{k, l}\right)=Q_{k, l} \quad \forall l, k>0$.

\section{Demonstração:}

Vamos provar a afirmação fazendo indução no número de células e no número de linhas.

Para $k=1$, temos $\mu_{1, l}=d_{l}$ e portanto, pela Proposição anterior, $\theta\left(\mu_{1, l}\right)=Q_{1, l}$. Assumimos portanto o resultado válido para todo diagrama $\mu_{i, j}$ tal que $i+j-1<m$ 
(isto é, tenha menos de $m$ células) e também para todo diagrama $\mu_{i, j}$ com exatamente $m$ células e tal que $i \leq k$ (isto é, com no máximo $k$ linhas). Vamos mostrar então que o resultado vale para o diagrama $\mu_{k+1, m-k}$, que possui exatamente $m$ células e $k+1$ linhas.

Considere o produto $c_{k} \cdot d_{m-k}$ em $Y$. Como já vimos, esse produto é dado por:

$$
c_{k} \cdot d_{m-k}=\mu k+1, m-k+\mu_{k, m-k+1}
$$

O diagrama $\mu_{k, m-k+1}$, pela hipótese de indução é levado por $\theta$ em $Q_{k, m-k+1}$. Por outro lado, $\operatorname{como} \theta$ é homomorfismo de álgebras, temos:

$$
\theta\left(c_{k} \cdot d_{m-k}\right)=\theta\left(c_{k}\right) \cdot \theta\left(d_{m-k}\right)
$$

E, novamente pela hipótese de indução:

$$
\theta\left(c_{k} \cdot d_{m-k}\right)=Q_{k, 1} \cdot Q_{1, m-k}
$$

Sendo assim:

$$
\theta\left(\mu_{k+1, m-k}\right)=\theta\left(c_{k} \cdot d_{m-k}\right)-\theta\left(\mu_{k, m-k+1}\right)=Q_{k, 1} \cdot Q_{1, m-k}-Q_{k, m-k+1}
$$

Logo, pela Proposição 4.3.7, temos:

$$
\theta\left(\mu_{k+1, m-k}\right)=Q_{k+1, m-k} .
$$

Teorema 4.3.1 $\theta(\lambda)=Q_{\lambda}$, para todo diagrama de Young $\lambda$.

A demonstração por nós conhecida desse Teorema depende diretamente da relação entre o invariante $\chi_{N}$ e o $s l(N)$ - invariante quântico, dada pelo seguinte resultado:

Teorema 4.3.2 ([Anna], pg.89) Seja $L=L_{1} \cup L_{2} \cup \ldots \cup L_{k}$ link orientado de $k$ componentes. Então:

$$
J\left(L ; V_{\lambda_{1}}, \ldots, V_{\lambda_{k}}\right)=\chi_{N}\left(L_{1} * Q_{\lambda_{1}}, \ldots\right)
$$

onde $\operatorname{sl}(N)$-invariante quântico de $L$ colorido pelas representações $V_{\lambda_{i}}$. 
Mais especificamente, a prova depende da inversibilidade da matriz

$$
M=\left(M_{\lambda, \mu}\right)_{\lambda, \mu \in \operatorname{Diag}_{\mathrm{V}, r}}
$$

onde:

(i) $\operatorname{Diag}_{N, r}$ é um subconjunto finito (a ser definido no próximo capítulo) de $Y_{N}$;

(ii) $M_{\lambda, \mu}=\chi_{N}\left(H ; Q_{\lambda}, Q_{\mu}\right)$, sendo $H$ o link de Hopf.

Sabemos que essa matriz é inversível, pois vale que:

Lema 4.3.7 ([TW]) Seja $q=s^{2}$ uma $r$-ésima raiz primitiva da unidade e $N \in$ $\mathbb{N}^{*}$ fixo. Sejam $\lambda, \mu \in \operatorname{Diag}_{N, r}$. Seja $J\left(H ; V_{\lambda}, V_{\mu}\right)$ o sl $(N)$ - invariante quântico do link de Hopf colorido pelas representaçôes $V_{\lambda}$ e $V_{\mu}$. Considere $M$ a matriz dada por $\left(M_{\lambda, \mu}\right)_{\lambda, \mu \in \text { Diag }_{N, r}}$. Então $M$ é inversível. 


\section{Capítulo 5}

\section{Considerações finais: $\mathrm{O}$ invariante $T(L)$}

\section{Introdução:}

Mostraremos nesse capítulo que a partir do invariante de links orientados $\chi_{N}$, calculado em raízes primitivas da unidade, pode-se construir um invariante $T(L)$ topológico de variedades de dimensão 3 compactas, conexas e orientáveis, usando decorações por elementos especiais $\Omega_{r}$. Ressaltamos que apenas daremos a definição de $T(L)$ não sendo dada a prova da sua invariância sob os movimentos de Fenn-Rourke (podendo ser encontrada em [Anna]). O motivo para isto, como já foi colocado na Introdução deste trabalho, é que a maioria dos resultados envolvem a Teoria de Representações da álgebra de Lie $\operatorname{sl}(N)$ e propriedades do $\operatorname{sl}(N)$-invariante quântico.

Lembramos que as decorações são feitas com elementos de $C^{+}$que mostramos ser isomorfo a $R_{\infty}$, o anel de polinômios em um número infinito e enumerável de indeterminadas e ao anel dos diagramas de Young $Y$. Explicitamos esse isomorfismo mostrando que cada diagrama de Young $\lambda$ corresponde ao fecho do idempotente $\frac{1}{\alpha_{\lambda}} e_{\lambda} \in H_{|\lambda|}$, o elemento $Q_{\lambda}$.

Fixado um $N>0$ e um $r>N$, definimos um novo parâmetro $q=e^{h}$. Fazendo $q$ uma $r$-ésima raiz primitiva da unidade, nosso espaço de decoração passa a ser um $\mathbb{C}$-espaço vetorial (antes apenas um $\Lambda$-módulo). Porém, ainda estamos lidando com dimensão infinita. O objetivo é, portanto, restringir nosso "campo de atuação" ao um espaço de dimensão finita. 
Obs: Não faremos distinção entre os espaços $C^{+}, R_{\infty}$ e $Y$, bem como subespaços correspondentes.

\section{$5.1 \mathrm{O}$ espaço $R_{N} / \mathcal{I}$}

Vamos primeiramente lembrar que começamos esse trabalho com o anel:

$$
\Lambda=\mathbb{C}\left[x^{ \pm 1}, v^{ \pm 1}, s^{ \pm 1}, z, \delta\right] /\left\langle v-v^{-1}=z \delta>\right.
$$

com $z=s-s^{-1}$, e após algumas substituições o nosso anel $\Lambda$ passou a ser o anel das séries formais $\mathbb{C} \llbracket h \rrbracket$, onde $s=e^{h / 2}$.

Seja $q=e^{h}$. Quando colocamos $q$ uma $r$-ésima raiz primitiva da unidade, o nosso anel $\Lambda$ passa a ser $\mathbb{C}$ e portanto $R_{\infty}$ passa a ser um espaço vetorial sobre esse corpo.

Definição 5.1.1 Fixemos então um $N>0$ e $r \in \mathbb{N}, r>N$, substituindo $q$ por uma $r$-ésima raiz primitiva da unidade. Definimos o nível $l$ de $q$ como $l=r-N$.

Dizemos que um diagrama é $\boldsymbol{q}$-admissível se possui menos que $N$ linhas e no máximo $l$ colunas. Seja $\operatorname{Diag}_{N, r}$ o conjunto de tais diagramas.

É imediato que $\operatorname{Diag}_{N, r} \subseteq R_{N}=\Lambda\left[c_{1}, \ldots, c_{N}\right]$. Seja $\mathcal{I}$ o ideal em $R_{N}$ gerado pelos diagramas de exatamente $l+1$ colunas.

Teorema 5.1.1 ([Anna], pg.146) $\operatorname{Diag}_{N, r}$ gera o espaço $R_{N} / \mathcal{I}$.

Considere agora o ideal $I \subseteq R_{N}$ gerado por $\left\{d_{r-1}, d_{r-2}, \ldots, d_{r-N+1}\right\}$, onde $d_{i}$ é o diagrama linha com $i$ células.

Proposição 5.1.1 Seja p tal que $l<p<r$. Então $\chi_{N}\left(Q_{1, p}\right)=0$.

\section{Demonstração:}

Pela Proposição 4.3.9, temos:

$$
\chi_{N}\left(Q_{1, p}\right)=\prod_{i=1}^{p} \frac{s^{N} s^{i-1}-s^{-N} s^{-(i-1)}}{s-s^{-i}}=s^{-(N+1) p} \prod_{i=1}^{p} \frac{q^{N+i-1}-1}{q-1}
$$


Quando $l<p<r$ e $1 \leq i \leq p$, lembrando que $l=r-N$, $\left(q^{i}-1\right)$ não se anula e portanto $\chi_{N}\left(Q_{1, p}\right)$ calculado em $q$ raiz $r$-ésima primitiva da unidade, está bem definido. Por outro lado, quando $i=l+1=r-N+1$, temos:

$$
q^{N+r-N+1-1}-1=q^{r}-1=0
$$

e portanto $\chi_{N}\left(Q_{1, p}\right)=0$.

Corolário 5.1.1 ([Anna], pg.150) Seja L um link com uma componente decorada por $d_{p}$, com $l<p<r$ e as restantes por $\lambda_{i}$, para $\lambda_{i} \in R_{N}$. Então, quando $q$ é uma raiz $r$ - ésima primitiva da unidade, $\chi_{N}(L)=0$.

Proposição 5.1 .2 ([Anna], pg.156) $I=\mathcal{I}$. Além disso Diag N,$r_{\text {é base para } o}$ $\mathbb{C}$-espaço vetorial $R_{N} / I$.

Obs: Os resultados acima justificam portanto a escolha do ideal $I$.

Definição 5.1.2 Seja $N \in \mathbb{N}^{*}$ fixado. Seja $\lambda=\left(\lambda_{1}, \lambda_{2}, \ldots, \lambda_{N}\right)$ um diagrama de Young. Seja $r_{i}=\lambda_{i}-\lambda_{i+1}$, para $i<N$ e $r_{N}=\lambda_{N}$. Temos que:

$$
\lambda=\left(\sum_{i=2}^{N} r_{i}, \sum_{i=2}^{N} r_{i}, \ldots, \sum_{i=N}^{N} r_{i}\right) .
$$

Definimos o diagrama dual de $\lambda$, denotado por $\lambda^{*}$, como:

$$
\lambda^{*}=\left(\sum_{i=1}^{N-1} r_{i}, \sum_{i=1}^{N-2} r_{i}, \ldots, r_{1}\right) .
$$

Pictoricamente, $\lambda^{*}$ é obtido ao removermos $\lambda$ do diagrama $\lambda_{1} \times N$ e rotacionarmos a figura restante em 180 graus.

Exemplo: $\begin{aligned} & \mathrm{V}=3 \\ & \nu^{*}=(3,2)\end{aligned}$


Observemos que, sendo $\lambda_{1}$ o número de colunas de $\lambda$, quando $\lambda \in \operatorname{Diag}_{N, r}$, temos $\lambda_{1} \leq l$. Logo:

$$
\lambda_{1}^{*}=\sum_{i=1}^{k} a_{i}=\lambda_{1}-\lambda_{k}<l
$$

Por outro lado, se $\lambda$ tem $m$ linhas, a representação pictórica de $\lambda^{*}$ nos diz que esse diagrama tem no máximo $m$ linhas. Além disso, é fácil ver que $\left(\lambda^{*}\right)^{*}=\lambda$. Concluimos então que:

$$
\lambda \in \operatorname{Diag}_{N, r} \Leftrightarrow \lambda^{*} \in \operatorname{Diag}_{N, r}
$$

Nota: As representações de $\operatorname{sl}(N)$ são indexadas pelos diagramas de Young, mais ainda, o anel das representações dessa álgebra é isomorfo à $Y_{N} /<c_{N}=1>$. Dessa teoria obtemos o seguinte resultado:

Teorema 5.1.2 [FH] Se $V_{\lambda}$ é uma representação irredutível de sl(N), então:

$$
\left(V_{\lambda}\right)^{*} \simeq V_{\lambda^{*}}
$$

Baseando-se nesse Teorema, é razoável que valha o seguinte fato (importante na prova de que $T(L)$ é um invariante sob os movimentos de Fenn-Rourke):

Proposição 5.1 .3 ([Anna $]$, pg.127) $\chi_{N}\left(Q_{\lambda}\right)=\chi_{N}\left(Q_{\lambda^{*}}\right)$,

Vamos assumir agora $\chi_{N}$ sempre calculado em $q$ raiz $r$-ésima primitiva da unidade. Seja:

$$
\Omega_{r}=\sum_{\lambda \in \operatorname{Diag}_{N, r}} \delta_{\lambda^{*}} \lambda
$$

onde $\delta_{\lambda^{*}}=\chi_{N}\left(Q_{\lambda^{*}}\right)$. Observemos que pela Proposição anterior, $\delta_{\lambda}=\delta_{\lambda^{*}}$.

Definição 5.1.3 Seja $L$ um $\operatorname{link}$ com $k$ componentes. Definimos a matriz $\left(l_{i j}\right)_{k \times k}$ onde:

$$
l_{i j}= \begin{cases}l k\left(L_{i}, L_{j}\right) & \text { para } j \neq i \\ \text { framing em } L_{i}, & \text { para } i=j\end{cases}
$$

Como $l k\left(L_{i}, L_{j}\right)=l k\left(L_{j}, L_{i}\right)$ (ver Capítulo 1 ), a matriz é simétrica e pode ser pensada como uma forma quadrática. Definimos $\operatorname{sig}(L)$ como a assinatura dessa forma. 
Seja $F_{\lambda} \in H_{|\lambda|}$ o elemento:

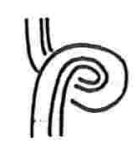

Proposição 5.1 .4 ([AM], pg.17) $O$ elemento $F_{\lambda}$ é central em $H_{|\lambda|}$ e além disso:

$$
e_{\lambda} F_{\lambda}=F_{\lambda} e_{\lambda}=f_{\lambda} e_{\lambda}
$$

onde $f_{\lambda} \in \Lambda$ é inversível.

Nota: Observe que o elemento $F_{\lambda}$ está diretamente relacionado com os movimentos de Fenn-Rourke, vindo daí portanto, a sua importância na definição do invariante $T(L)$.

Considere agora os seguinte escalares:

$$
\begin{gathered}
c_{+}=\sum_{\nu \in \operatorname{Diag}_{N, r}} f_{\nu} \delta_{\nu}^{2} \\
c_{-}=\sum_{\nu \in \operatorname{Diag}_{N, r}} f_{\nu}^{-1} \delta_{\nu}^{2}
\end{gathered}
$$

Observamos que $c_{+}$(respectivamente, $c_{-}$) é o valor de $\chi_{N}$ no nó trivial com framing +1 (resp. -1 ) decorado por $\Omega_{r}$. Como $c_{+} \in \mathbb{C}$, podemos escrevê-lo como o produto de um número real positivo $\rho(r)$ e um número complexo de norma unitária $c(r)$, isto é $c_{+}=\rho(r) c(r)$.

Finalmente, chegamos à definição do invariante de 3-variedades $T(L)$ :

Teorema 5.1.3 ([Anna], pg.162) O elemento:

$$
T(L)=\rho(r)^{-k} c(r)^{-s i g(L)} \chi_{N}\left(L ; \Omega_{r}, \ldots, \Omega_{r}\right)
$$

onde $k$ é o número de componentes de L, é invariante sob os movimentos de FennRourke. 


\section{Bibliografia}

[A] Artin, E. "Theory of braids." Ann.Math. 48 (1947) pp 1010-1026.

[Anna] Aiston, A. "Skein theoretic idempotents of Hecke algebras and quantum group invariants." Tese de Doutorado, Univ.Liverpool, 1986

[AM] Aiston, A. e Morton, H.R. "Idempotents of Hecke algebras of type A." (preprint) Univ.de Liverpool, (1996).

[Atiyah] Atiyah, M. "The Geometry and Physics of Knots." Cambridge Univ.Press (1990).

[Birman] Birman, J.. "Braids, links and mapping class groups." Annals of Math.Studies 82, Princeton Univ.Press (1975)

[BZ] Burde, G. e Zieschang, H. "Knots." Walter de Gruyter, (1997).

[Cohn] Cohn, P.M. "Algebra, vol.II" John Wiley \& Sons (1989)

[C] Conway, J. "An enumeration of knots and links." Computational problems in abstract algebra. Pergamon Press, New York (1970) pp 329-358.

[FR] Fenn, R. e Rourke, C. “On Kirby's calculus of links.” Topology 18 (1979) pp $1-15$.

[FH] Fulton, W. e Harris, J. "Representation theory - a first course." G.T.M. Springer Verlag (1991)

[FRT] Frame, J.S.; Robinson, G.de B. e Thrall, R.M. "The hook graphs of the symmetric group." Canad.J of Math. 6 (1954) pp 316-324.

[Go] Goldschmidt, D.M. "Group characters, symmetric functions and the Hecke algebra." Univ.Lecture Series, A.M.S. 4 (1993). 
[JL] James, G. e Liebeck, M. "Representations and characters of groups." Cambridge Univ.Press (1993).

[Jones] Jones, V.F.R. "Hecke algebra representations of braid groups and link polynomials." Ann.of Mathematics 126 (1987), pp 335-388.

[Kau1] Kauffmann, L.H. "On knots.” Ann.of Math.Studies, 115, Princeton Univ.Press (1987)

[Kau2] Kauffmann, L.H. "Formal knot theory" Mathematical Notes 30, Princeton Univ.Press (1983)

[Ki] Kirby, R. "A calculus of framed links in $S^{3}$." Invent.Math. 45 (1978) pp 35-56.

[Li1] Lickorish, W.B.R. "A representation of orientable, combinatorial 3-manifolds." Ann.Math. 76 (1962) pp 531-540.

[Li2] Lickorish, W.B.R. "Linear Skein theory and link polynomials." Topology and its Applications 27 (1987) pp 265-274.

[MS] Morton, H. e Short, H.B. "The 2-variable polynomial of cable knots." Math.Proc.Camb.Philos.Soc. 101 (1987), pp 267-278.

[MT] Morton, H. e Traczyc, P. "Knots and algebras." Contribuciones Matematicas en homenaje al professor D.Antonio Plans Sanz de Bremond, Ed.E.Martin-Peinador e A.Rodez Usan. Univ.de Zaragoza (1990) pp 201-220.

[PS] Prasolov, V.V. e Sossinsky, A.B. "Knots, links, braids and 3-manifolds: An introduction to the new invariants in low-dimensional topology." Translations of Math.Monographs, A.M.S. 154 (1996).

[RT2] Reshetikhin, N. Yu. e Turaev, V.G. "Invariants of 3-manifolds via link polynomials and quantum groups." Invent.Math. 103 (1991) pp 547-597.

[Rol] Rolfsen, D. "Knots and links." Publish or Perish Inc. (1976).

[Turaev] Turaev, V.G. "The Conway and the Kauffman modules of the solid torus with an appendix on the operator invariants of tangles." L.O.M.I. pre-prints E6-88 (1988).

[TW] Turaev, V.G. e Wenzl, H. "Quantum invariants of 3-manifolds associated with classical semi-simple Lie algebras." Int.J.Math. 4 (1993) pp 323-358.

[WZ] Wenzl, H. "Hecke algebras of type $A_{n}$ and sub-factors" Invent.Math. 92 (1988), pp 349-383 
[Y] Yokota, Y. "Skeins and quantum SU(N) invariants of 3-manifolds." Math.Ann. 307 (1997), pp 109-138. 